\title{
Open Access et bibliothèques
}

Étude réalisée par

\section{Alain \\ Caraco}

Conservateur général

des bibliothèques

19 décembre 2018

(CC BY-NC-SA 4.0)

enssib 

Open Access et bibliothèques

Alain Caraco

Version 1.2 - 19 décembre 2018

(CC BY-NC-SA 4.0) 


\section{Science ouverte et bibliothèques : une chance à saisir, une chance saisie}

De « l'Initiative de Budapest pour l'accès ouvert », en 2002, à « l'Appel de Jussieu pour la science ouverte », en 2017, la route menant au libre accès aux travaux et aux résultats de la recherche scientifique, toutes disciplines confondues, a été longue, semée d'embûches et d'épines. Marin Dacos, aujourd'hui conseiller pour la science ouverte au ministère chargé de l'enseignement supérieur et de la recherche, le reconnaît lui-même, d'ailleurs sans amertume ni découragement : «Je pensais qu'en cinq ans j'aurais réussi, mais ça a été plus long ${ }^{1}{ }^{1}$ Et si, en France, un pas décisif a été fait en 2018, il reste encore beaucoup de chemin à parcourir.

Pour les bibliothèques, les premières à avoir été touchées de plein fouet par l'augmentation exponentielle des tarifs d'accès aux publications scientifiques en ligne, les premières à avoir réagi collectivement, avec la mise en place des consortiums, la prise de conscience a certes permis de lancer des actions destinées à enrayer le manège infernal des abonnements prohibitifs et des stratégies de fermeture des éditeurs spécialisés mais, trop souvent ignorées ou marginalisées par les milieux de la recherche, les services documentaires ont dû batailler ferme pour s'imposer dans le débat et se faire reconnaître comme des acteurs légitimes du combat pour la science ouverte. Et cette reconnaissance reste fragile, dans un écosystème bouleversé où la documentation et l'information scientifique et technique, carburants quotidiens de la recherche, semblent, à l'heure d'Internet et du numérique « total $»^{2}$, échapper aux bibliothèques et aux bibliothécaires.

J'ai donc accueilli avec beaucoup d'intérêt, au printemps 2017, la proposition d'Alain Caraco, directeur du service commun de la documentation de l'université Savoie Mont Blanc, de venir à l'Enssib dans le cadre d'un congé de formation d'un an, précisément consacré à l'Open Access. Son projet poursuivait deux objectifs, comme il le précise lui-même dans son avant-propos : compléter ses connaissances sur le sujet, et mener un travail de recherche et de propositions susceptible d'être utile à la large communauté professionnelle des bibliothèques et services documentaires. Si la réalisation du premier objectif ne relève que de l'évaluation personnelle de la formation suivie, le second s'est matérialisé dans le présent rapport, travail exemplaire que je suis très heureux de présenter au public de l'Enssib et, au-delà, aux communautés de la recherche et de la science.

1. David Larousserie. « Marin Dacos, héraut de la science ouverte». In : Le Monde (4 déc. 2018). URL : https : / / ww . lemonde .fr/sciences /article/2018/12/04/marin-dacos-heraut-de-lascience-ouverte_5392481_1650684.html (visité le 05/12/2018).

2. «total» et non " généralisé » : tout doit être numérique, rien ne peut plus exister en-dehors, ce n'est qu'une question de temps (le temps de la généralisation, justement). 
Le rapport est en effet exemplaire, au moins à deux titres : d'abord par son approche extrêmement concrète et pratique, en second par sa volonté, sinon d'épuiser le sujet (il est en réalité inépuisable), du moins d'en faire un tour aussi complet que possible. Ne retrouve-t-on pas dans cette approche la « sagesse du bibliothécaire $»,{ }^{3}$ sa modestie et son orgueil : modestie de n'être qu'un intermédiaire, un passeur, un ouvreur de portes; orgueil d'être capable d'aller au bout d'un sujet, d'une question, en passant tout, jusqu'au détail le plus infime, au tamis de la vérification. En ces temps de vérité bafouée ou niée, cette sagesse-là est plus que jamais précieuse.

Mais les chemins de la sagesse sont multiples et se croisent de façon inopinée. Alain Caraco a rédigé son rapport au printemps 2018, au moment même où les pouvoirs publics optaient d'une manière non ambiguë pour un changement d'approche radical. Promulguée le 7 octobre 2016, la Loi pour une République numérique, dite loi Lemaire, retranscrite depuis dans le Code de la recherche (article L533-4), avait déjà autorisé les chercheurs dont l'activité est financée au moins pour moitié sur fonds publics à déposer leur article dans une archive ouverte, au plus tard un an après la publication par l'éditeur. Allant beaucoup plus loin - mais quel temps il avait fallu pour en arriver là! - en janvier 2018, lors des journées nationales de la science ouverte, Alain Beretz, directeur général de la recherche et de l'innovation, déclarait que le ministère avait la volonté de saisir l'opportunité de la science ouverte à bras le corps et que la ministre et lui-même étaient pro-actifs et assez enthousiastes. Et en effet, le 4 juillet suivant, Frédérique Vidal, dans un discours prononcé au Lilliad Learning Center Innovation de Lille, lors du congrès de la Ligue européenne des bibliothèques de recherche, annonçait le lancement d'un ambitieux Plan national pour la science ouverte. ${ }^{4}$ Plan qui apparaissait comme un filigrane, ou plutôt comme un fantôme dont il eût espéré plutôt que redouté l'approche, dans les versions successives du rapport d'Alain.

Jugez-en. Dans ce texte, que vous allez découvrir, Alain Caraco fait dix-huit propositions pour faire avancer la science ouverte. Les propositions 1 à 12 traitent de l'Open Access en général et leur contenu se retrouve tout naturellement dans les projets et actions du plan gouvernemental, aujourd'hui mis en œuvre par le Comité pour la science ouverte (CoSO), successeur de la Bibliothèque scientifique numérique. Les propositions 13 à 17 concernent directement les services communs de documentation et les bibliothèques. Leur mise en œuvre dépendra beaucoup de leur appropriation par la communauté professionnelle. Enfin, la dix-huitième proposition, la plus ambitieuse, et sans doute la première à avoir émergé dans la réflexion de l'auteur, qui me l'a fait partager dès la fin de 2017, imagine une « plateforme fédératrice de l'action des bibliothèques pour l'Open Access en France ». Belle idée, mais entretemps, le Comité pour la science ouverte - et on ne peut que s'en réjouir - se mettait en marche pour ouvrir précisément, en cette fin de 2018, un portail national de la science ouverte : le site " Ouvrir la science ${ }^{5}{ }^{5}$ La voilà, cette plateforme, et plus large que si elle eût desservi seulement la communauté bibliothécaire, car travaillant sur des axes thématiques en s'appuyant sur les collèges du comité et en fédérant veille et ressources dans une approche transversale.

Pour cette plateforme nationale, comme vous le verrez dans la description détaillée qu'il en fait, pages 74 et 75, Alain Caraco proposait un portage par l'Université de Lyon

3. Michel Melot. La sagesse du bibliothécaire. Sagesse d'un métier. Paris : l'Oeil neuf éd., 2004, 109 p. ISBN : 978-2-915543-03-2. URL : http://www.sudoc.fr/077485920.

4. Plan national pour la science ouverte : discours de Frédérique Vidal - ESR : enseignementsuprecherche.gouv.fr. URL : http://www.enseignementsup-recherche.gouv.fr/cid132531/plan-nationa 1-pour-la-science-ouverte-discours-de-frederique-vidal.html (visité le 06/07/2018).

5. CoSO. Ouvrir la Science. URL : https://www.ouvrirlascience.fr/ (visité le 10/12/2018). 
ou / et par l'Enssib. Cette hypothèse, on l'aura compris, n'est plus d'actualité. Mais, compte tenu de l'intérêt de cette proposition sur de nombreux plans, veille documentaire, formation, observatoire des actions portées par les bibliothèques, le CoSO a proposé à l'Enssib d'intégrer le comité de rédaction du site « Ouvrir la science ». L'école saura, dans une démarche collégiale et largement ouverte, relever le défi de cette participation, dès le début de 2019, et l'auteur du rapport Open Access et bibliothèques y aura évidemment toute sa part. Ce ne sera que justice, car un tel travail, mis sans conditions à la disposition de tous, est, comme je crois l'avoir déjà dit, mais il ne faut pas craindre de se répéter, œuvre de sagesse.

Yves Alix 


\section{Sommaire}

$\begin{array}{ll}\text { Préface } & 3\end{array}$

$\begin{array}{lr}\text { Sommaire } & 7\end{array}$

$\begin{array}{lr}\text { Synthèse } & 9\end{array}$

$\begin{array}{lr}\text { Avant-propos } & 15\end{array}$

$\begin{array}{ll}\text { Remerciements } & 17\end{array}$

$\begin{array}{lr}\text { Introduction } & 19\end{array}$

Un coût qui n'est plus soutenable . . . . . . . . . . . . . . . . . . 19

Un modèle économique obsolète . . . . . . . . . . . . . . . . . . . 20

Un grand intérêt pour l'OA . . . . . . . . . . . . . . . . . 21

1 Open Access : de quoi parle-t-on? $\quad 25$

1.1 Green $\mathrm{OA}$ : la voie verte . . . . . . . . . . . . . . . 25

1.2 Gold OA : la voie dorée . . . . . . . . . . . . . . . . . 30

1.3 Diamant et platine : l'édition électronique en OA . . . . . . . . . . 34

1.4 D'autres formes de revues . . . . . . . . . . . . . . . 38

1.5 Grey : la zone grise . . . . . . . . . . . . . . . . . . . 40

1.6 Black : les solutions illégales . . . . . . . . . . . . . . . . . . 41

1.7 De l'Open Access à la Science ouverte . . . . . . . . . . . . . . . . . 43

2 Etat des lieux $\quad 47$

2.1 HAL et les archives ouvertes institutionnelles . . . . . . . . . . . . 47

2.2 Initiatives académiques en faveur de l'OA . . . . . . . . . . . . . . 50

2.3 Manifestations à l'occasion de l'Open Access Week . . . . . . . . . . . . . 54

2.4 Journées d'étude . . . . . . . . . . . . . . . . . . . 55

3 Obstacles $\quad \mathbf{5 7}$

3.1 C'est quoi, les archives ouvertes? . . . . . . . . . . . . . . 57

3.2 Y ai-je intérêt $\ldots \ldots \ldots \ldots \ldots \ldots \ldots \ldots$

3.3 Ai-je le droit $? \ldots \ldots$. . . . . . . . . . . . . . . . . . . . . . . . . . . 59

3.4 C'est trop compliqué / Je n'ai pas le temps . . . . . . . . . . . . . . 60

3.5 Je trouve déjà ce que je cherche ailleurs . . . . . . . . . . . . . . . 60

3.6 Je ne sais pas où déposer mes publications . . . . . . . . . . . . . . . 63

3.7 J'aimerais publier dans une revue en OA, mais je ne sais pas comment m'y prendre . . . . . . . . . . . . . . . . . . . 64 
4.1 Communiquer largement sur les objectifs et possibilités de diffusion des travaux de la recherche . . . . . . . . . . . . . . . . 65

4.2 Rendre la publication en OA incitative pour la carrière des chercheurs . . 67

4.3 Accélérer la prise en compte de l'OA par les établissements . . . . . . . . 71

4.4 Renforcer les services communs de documentation (SCD) dans leur rôle d'acteur du développement de l'OA . . . . . . . . . . . . . . 72

4.5 Ajouter des fonctionnalités complémentaires à HAL . . . . . . . . . 73

4.6 Créer une plateforme fédératrice de l'action des bibliothèques pour l'Open Access en France . . . . . . . . . . . . . . . . . . . . . 74

$\begin{array}{lr}\text { Conclusion } & 77\end{array}$

$\begin{array}{ll}\text { Résumé des propositions } & 79\end{array}$

$\begin{array}{lr}\text { Annexes } & 81\end{array}$

A Données : recensement des archives ouvertes des établissements d'enseignement supérieur et de recherche et des organismes de recherche 83 Méthode de constitution du corpus . . . . . . . . . . . . . . . . 83

A.1 Universités . . . . . . . . . . . . . . . . . . . . . 85

A.2 ComUEs et associations . . . . . . . . . . . . . . . . . 94

A.3 Instituts et écoles extérieurs aux universités . . . . . . . . . . . . . . . 96

A.4 Ecoles normales supérieures . . . . . . . . . . . . . . . . . . . . . 99

A.5 Grands établissements . . . . . . . . . . . . . . . . . . . . . . . 99

A.6 Ecoles nationales (supérieures) d'ingénieurs . . . . . . . . . . . . . . 104

A.7 Instituts d'études politiques . . . . . . . . . . . . . . . . . . . 104

A.8 Organismes de recherche . . . . . . . . . . . . . . . . . . 105

A.9 Bibliothèque nationale de France . . . . . . . . . . . . . . . . . . 110

B Données : recensement de l'OA week $2017 \quad 111$

Méthode de constitution du corpus . . . . . . . . . . . . . . . . . . 111

Evénements . . . . . . . . . . . . . . . . . . . . . . . . . . 112

$\begin{array}{ll}\text { C Extrait des documents stratégiques de l'Enssib } & 117\end{array}$

C.1 Contrat de site 2016-2020 Université de Lyon : volet spécifique Enssib . . 117

C.2 Projet d'établissement Enssib 2020 . . . . . . . . . . . . . . . 117

Glossaire

Bibliographie 


\section{Synthèse}

Confrontés à une explosion du coût de la documentation scientifique depuis le début du XXI ${ }^{e}$ siècle, le monde de l'enseignement supérieur et de la recherche (ESR) cherche des solutions pour concilier accès à l'information et soutenabilité économique.

Enjeu majeur des années à venir, l'Open Access (OA) peut constituer une alternative crédible à l'offre éditoriale concentrée entre les mains d'une poignée d'éditeurs transnationaux.

\section{Open Access : de quoi parle-t-on?}

Pendant longtemps, on a pu se contenter de parler des deux voies de l'Open Access (OA), la voie verte, ou "Green OA" et la voie dorée, ou "Gold OA", définies en 2002 dans l'Initiative de Budapest. Pourtant, au fil du temps, le paysage s'est fortement complexifié.

Green OA : la voie verte La voie verte repose sur l'auto-archivage par les chercheurs de leurs propres publications, sur des serveurs par leur communauté, appelés Archive ouverte (AO). On distingue les archives ouvertes thématiques, contrôlées par une communauté de chercheurs (arXiv et ses dérivées, PubMed Central, RePEc...), des archives ouvertes institutionnelles (AOI), créées à l'initiative d'un établissement.

La voie verte est privilégiée par la Belgique, le Luxembourg, l'Espagne et l'Amérique latine.

En France, HAL représente un cas unique au monde d'archive ouverte nationale pluridisciplinaire, qui propose aux établissements qui le souhaitent de créer des portails, qui, de fait, tiennent lieu d'archive ouverte institutionnelle.

Gold OA : la voie dorée A l'origine, la voie dorée ne définissait pas un modèle économique, mais seulement les revues à comité de lecture immédiatement et gratuitement accessibles en ligne. Dans les faits, la majorité de revues en Gold OA demandent aux auteurs ou à leur institution les paiement d'Article Processing Charge (APC), qui varient selon le prestige de la revue, la moyenne se situant un peu au dessous de $2000 €$. On appelle Bronze OA une version dégradée du Gold OA.

La voie dorée est privilégiée par le Royaume-Uni, Les Pays-Bas, l'Allemagne, la Finlande et la Suisse.

Diamant et platine : l'édition électronique en OA On appelle Diamond OA ou Platinium OA l'édition de revues en $\mathrm{OA}$ sans APC, généralement à l'initiative de structures publiques ou coopératives. Leur modèle économique peut combiner le financement public et le freemium (service de base gratuit et services complémentaires 
payants). Quelques exemples : OpenEdition, Persée, EDP Sciences et quelques Universités (France), Erudit (Québec), SciELO et Redalyc (Amérique latine), Fair open access alliance...

Les zones grise et noire Les réseaux sociaux académiques (Academia.edu et ResearchGate) permettent aux chercheur d'y déposer les PDF de leurs publications, mais n'offrent pas les garanties des archives ouvertes, en particulier en terme de pérennité.

Il existe des outils de recherche (Unpaywall, Lazy Scholar, Open Acces Button, Kopernio) qui sont utiles pour accéder librement à l'information scientifique, mais à condition que celle-ci ait été au préalable publiée en OA par un tiers. Ils ne dispensent donc pas les chercheurs et les institutions de leur effort pour le développement de l'OA.

Enfin, Sci-Hub et LibGen, très riches et très utilisées y compris dans les pays développés, sont des archives ouvertes pirates. Selon le point de vue, elles peuvent être perçues comme une atteinte au droit légitime de propriété intellectuelle ou comme une entreprise mondiale d'émancipation de la science. Bien évidemment, aucun pays ou institution ne peut en faire la base d'une politique d'OA.

De l'Open Access à la Science ouverte La science ouverte est le prolongement naturel de l'Open Access. Elles est la généralisation à toutes les étapes de la production scientifique des principes de l'OA. Les bibliothèques sont particulièrement concernées par le travail collaboratif sur les preprints, la publication des données de la recherche, la citation ouverte et l'évaluation ouverte. Depuis 2017, un poste de conseiller scientifique pour la Science Ouverte a été créé auprès du DGRI et confié à Marin Dacos, le fondateur d'OpenEdition. En mars 2018, la Bibliothèque scientifique numérique (BSN) s'est transformée en Comité pour la Science ouverte (CoSO). Enfin, le 4 juillet 2018, la ministre Frédérique Vidal a lancé un plan national pour la science ouverte.

\section{Etat des lieux}

Afin d'avoir une vision du développement de l'OA dans le monde de l'ESR français, l'état des lieux étudie les portails HAL et les archives ouvertes institutionnelles, ainsi que les initiatives académiques en faveur de l'OA.

\section{HAL et les archives ouvertes institutionnelles}

Le recensement des archives ouvertes a été effectué fin 2017 et actualisé fin 2018 pour 195 établissements d'enseignement supérieur et de recherche, d'associations de sites universitaires et d'organismes de recherche.

Depuis plus de 10 ans, l'ouverture de portails dans HAL et la création d'AOI se poursuivent de manière constante et linéaire. L'usage de HAL, sous la forme de portail (49\%) ou de collection (19\%), est prédominant sur l'ensemble du corpus. Seuls $9 \%$ ont une AOI et $23 \%$ des établissements étudiés n'avaient pas d'archive ouverte à la date du recensement. Certains en avaient cependant le projet à plus ou moins brève échéance.

\section{Initiatives académiques en faveur de l'OA}

A côté des dépôts d'archives ouvertes, certains établissements d'ESR ont pris d'autres initiatives en faveur de l'OA. 
Le mandat de libre accès est l'obligation faite par une institution à ses membres de publier leur production scientifique en OA, soit dans un dépôt d'archive ouverte, soit dans une revue en $\mathrm{OA}$, soit les deux à la fois. Une dizaine d'établissements français, dont trois universités, l'ont adopté.

Plusieurs établissements proposent des services spécifiques pour développer l'OA, auquel il faut ajouter le Groupe de travail accès ouvert de Couperin, qui est la seule initiative mutualisée.

Le rapport recense également les manifestations à l'occasion de l'Open Access Week et les journées d'étude consacrées à l'OA.

\section{Obstacles}

Le développement de l'OA se heurte à de nombreux obstacles invoqués par les chercheurs. Il sont généralement dus à leur méconnaissance du fonctionnement de l'OA. Afin de fournir aux bibliothécaires des outils pour lever ces obstacles, un argumentaire propose des réponses aux questions et objections fréquemment posées par les chercheurs :

- C'est quoi, les archives ouvertes?

- Je ne veux pas divulguer ma production sans contrôle

- Est-ce bon pour ma carrière?

- La publication en OA n'offre pas la garantie de qualité de l'édition commerciale

- HAL est encore un de ces machins franco-français : je préfère déposer mes publications sur Academia ou ResearchGate pour avoir une vraie visibilité internationale

- Ai-je le droit?

- C'est trop compliqué / Je n'ai pas le temps

- Je trouve déjà presque tout ce que je cherche sur gratuitement sur Internet

- Je trouve déjà ce que je cherche sur Academia / ResearchGate

- Unpaywall / Lazy Scholar / Open Access Button / Kopernio me permet de trouver gratuitement ce que je cherche

- Il suffit de demander sur Twitter avec le hastag \#IcanHazPDF

- Il y a tout sur SciHub et LibGen

- Je publie déjà dans des revues en OA (attention aux revues hybrides et aux revues prédatrices)

- Je ne sais pas où déposer mes publications (HAL, archive ouverte institutionnelle, archive ouverte thématique?)

— J'aimerais publier dans une revue en OA, mais je ne sais pas comment m'y prendre

\section{Propositions}

Face aux obstacles évoqués dans le chapitre précédent, une liste de mesures est proposée pour les aplanir.

\section{Communiquer largement sur les objectifs et possibilités de diffu- sion des travaux de la recherche}

1. Rappeler l'article L112-1 du code de la recherche, qui traite des objectifs de la recherche publique, dont font partie le partage et la diffusion des connaissances et des données scientifique, en priorité à des formats libres d'accès. 
2. Rappeler l'article L533-4 du code de la recherche, qui donne aux chercheurs le droit de déposer leurs écrits scientifiques en archive ouverte.

3. Rappeler que le programme européen de financement de la recherche H2020, qui comporte l'obligation d'assurer le libre accès aux publications issues des recherches auxquelles elle aura attribué un financement, même partiel, en Green ou en Gold OA.

4. Mener des campagnes nationales de communication régulières, notamment à l'occasion de l'Open Access Week, sur les établissements ayant adopté un mandat de libre accès et/ou créé des services autour de l'OA.

\section{Rendre la publication en OA incitative pour la carrière des cher- cheurs}

5. Sensibiliser chacune des sections du CNU afin qu'elles intègrent la publication en OA dans leurs critères d'évaluation.

6. Engager le dialogue entre le MESRI et le HCERES pour qu'il considère l'OA comme un critère important d'évaluation.

7. Inciter l'ANR à faire de la publication en $\mathrm{OA}$ une obligation pour les projets qu'elle finance, à l'instar de H2020.

8. Inciter les conférences d'établissements (CPU, CDEFI et CGE) à mettre en place un dispositif d'accompagnement de leurs établissements membres pour qu'ils formalisent et mettent en oeuvre leur propre politique d'OA.

9. Suivre le déroulement des travaux du groupe-projet « Évaluation de la Recherche » du CoSO et prendre des mesures opérationnelles à partir de ses propositions.

10. Etudier la transposition à l'évaluation des chercheurs français du rapport de la Commission européenne Evaluation of Research , Careers fully acknowledging, Open Science practices

\section{Accélérer la prise en compte de l'OA par les établissements}

11. Lancer un appel à projet MESRI pour un label « Université promotrice de l'Open Access ».

12. Rendre la définition d'une politique d'OA obligatoire pour les établissements d'enseignement supérieur et de recherche et les organismes de recherche

\section{Renforcer les services communs de documentation (SCD) dans leur rôle d'acteur du développement de l'OA}

13. Soutenir les SCD dans leur rôle d'accompagnateur au développement de l'OA, en créant dans chaque SCD une équipe dédiée et formée à l'OA.

14. S'appuyer sur les SCD pour garantir la qualité des métadonnées et des référentiels.

\section{Ajouter des fonctionnalités complémentaires à HAL}

15. Introduire des savoir-faire répandus dans le monde des bibliothèques pour la gestion des référentiels. 
16. Produire les données de l'ESGBU avec le module statistique de HAL.

17. Faire une revue systématique de l'interface de HAL.

\section{Créer une plateforme fédératrice de l'action des biblio- thèques pour l'Open Access en France}

18. Porte d'entrée de l'OA pour les bibliothèques de France, cette plateforme ferait principalement de la veille et de l'agrégation de ressources validées. Subsidiairement, la plateforme pourrait aussi produire de nouvelles ressources et améliorer des ressources existantes. La plateforme comprendrait aussi un observatoire de l'OA en France, base de données servant également de source à la publication d'un état des lieux annuel. 


\section{Avant-propos}

Directeur de bibliothèques (BDP, BM, BU) depuis trente ans, j'ai demandé et obtenu un congé de formation ${ }^{6}$ pour la durée de l'année universitaire 2017-2018, afin d'orienter ma carrière vers une activité plus scientifique. Ce congé a lieu à l'Enssib, sous la direction pédagogique et scientifique de son directeur, Yves Alix.

L'Open Access $(\mathrm{OA})^{7}$, sous ses deux formes « verte » et « dorée », est un enjeu majeur des années à venir. Face à l'explosion du coût de la documentation numérique, l'OA peut constituer une alternative crédible à l'offre éditoriale concentrée entre les mains d'une poignée d'éditeurs transnationaux. Dans un monde académique où prédominent les classements internationaux, l'OA donne une visibilité nécessaire à la recherche française.

Ces enjeux ont fait l'objet d'une prise de conscience accélérée depuis l'année 2016. La Stratégie nationale des infrastructures de recherche a reconnu HAL comme une des quatre infrastructures de recherche en IST. Les professionnels de la documentation ont créé leur club utilisateurs, CasuHAL. La loi 2016-1321 pour une République numérique, dite « Loi Lemaire » a, dans son article 30, levé un obstacle juridique, en accordant aux auteurs dont la recherche est financée au moins pour moitié par des crédits publics le droit de publier en archive ouverte la version finale de leur manuscrit acceptée pour publication, même après avoir accordé des droits exclusifs à un éditeur. Les premiers résultats de l'enquête COPIST, montrent un fort intérêt des répondants pour HAL.

Pourtant l'OA et le monde des bibliothèques d'enseignement supérieur et de recherche ne se connaissent pas assez et pourraient s'enrichir mutuellement en se rapprochant. Ma longue expérience du monde des bibliothèques et mon intérêt déjà ancien pour internet et l'accès libre à la connaissance m'ont laissé entrevoir que je pourrais être utile à la profession en me perfectionnant dans le domaine de l'OA.

Avec Yves Alix, nous avons établi le programme de cette année de formation en deux parties. La première a consisté à suivre des stages et des cours permettant de compléter mes connaissances sur l'OA. La deuxième est un travail de recherche et de propositions sur le même sujet. Le présent rapport en est le résultat. Dans l'esprit de l'OA, il a été écrit autant que possible à partir de sources récentes et librement accessibles en ligne, notamment des billets de blogs et des présentations dans des journées d'étude. Réalisé dans un temps limité, il ne prétend pas épuiser le sujet, qui est en ébullition permanente. Il a en revanche été conçu pour servir de base à une véritable boîte à outils vivante, à actualiser et à enrichir de manière coopérative.

6. article 30 du décret 92-26 du 9 janvier 1992 portant statut particulier du corps des conservateurs des bibliothèques et du corps des conservateurs généraux des bibliothèque

7. Bien qu'il existe la traduction française "Accès ouvert", celle-ci reste peu employée. Nous emploierons donc la forme anglaise et son abréviation dans le présent document. 


\section{Remerciements}

Je tiens à exprimer ma gratitude aux personnes grâce auxquelles j'ai pu passer l'année universitaire 2017-2018 à apprendre, approfondir et transmettre sur la question de l'Open Access, que je n'avais pu qu'approcher trop rapidement dans l'exercice de mes fonctions.

Yves Alix, directeur de l'Enssib, a accepté de m'accueilir en congé spécial de formation dans son école et d'assurer la direction pédagogique et scientifique de mon travail. Ses avis, conseils et encouragements m’ont été précieux.

Eric Guichard, maître de conférences HDR à l'Enssib, m'a permis de passer de vagues notions sur le langage éditorial $\mathrm{LAT}_{\mathrm{E}} \mathrm{X}$ à une pratique régulière. J'ai utilisé $\mathrm{IAT}_{\mathrm{E}} \mathrm{X}$, en combinaison avec Zotero, pour structurer et écrire le présent rapport et gérer ses références. Mieux encore, $\mathrm{HAT}_{\mathrm{E} X}$ est devenu maintenant mon outil favori pour rédiger un document un tant soit peu complexe.

Et tout le personnel de l'Enssib, qui m'a accueilli avec chaleur, facilité l'organisation de mon travail et offert des échanges d'idées fructueux à l'occasion de discussions improvisées.

Un grand merci à toutes et à tous! 


\section{Introduction}

Confrontés à une explosion du coût de la documentation scientifique depuis le début du XXI ${ }^{\mathrm{e}}$ siècle, le monde de l'enseignement supérieur et de la recherche (ESR) cherche des solutions pour concilier accès à l'information et soutenabilité économique.

Enjeu majeur des années à venir, l'Open Access (OA) peut constituer une alternative crédible à l'offre éditoriale concentrée entre les mains d'une poignée d'éditeurs transnationaux.

\section{Un coût qui n'est plus soutenable}

La transformation numérique de l'édition scientifique, à partir des dernières années du $\mathrm{XX}^{\mathrm{e}}$ siècle a changé son modèle de diffusion.

Les bibliothèques d'ESR étaient auparavant abonnées à des revues qu'elles choisissaient titre à titre, en fonction des besoins de leur communauté et de leur budget. Elle se sont progressivement retrouvées face à une offre sous forme de bouquets, au sein desquels un éditeur regroupait toutes ses revues d'un même champ disciplinaire, voire l'intégralité de ses revues. Cette évolution de l'offre présentait des avantages notables : pour quelques pourcents plus cher, une université de taille moyenne pouvait ainsi offrir à ses chercheurs quelques dizaines de milliers de revues au lieu de quelques centaines. De plus, plusieurs personnes pouvaient lire en même temps le même article, ce qui était impossible avec un abonnement papier. Enfin, l'accès à tous les articles était possible depuis tous les postes de travail de l'établissement, et souvent même de tout poste de travail distant d'une personne autorisée.

Les inconvénients de cette mutation sont apparus progressivement. Pour mémoire, on mentionnera la surabondance de l'offre documentaire par rapport à la capacité des chercheurs à l'utiliser : si le numérique permet de trouver dix ou cent fois plus de références dans un temps donné, le temps nécessaire pour les lire, les comprendre et en faire usage restera, lui, dix ou cent fois plus important. Plus grave, l'offre s'est concentrée entre les mains d'un nombre éditeurs restreints. Et ces derniers ont rapidement augmenté leurs prix au delà du raisonnable, puis du soutenable, face à un clientèle devenue dépendante. En 2014, la marge des six principaux éditeurs ${ }^{8}$ était comprise entre 24 et $39 \%$ ! $^{9}$ Ces

8. Reed-Elsevier (devenu RELX en 2015), Macmillan/Nature + Springer, Wiley, Thomson Reuters, Wolters Kluwer et Informa (Taylor \& Francis).

9. CNRS. « Résultats 2014 des grands éditeurs scientifiques : une croissance satisfaisante, des profits record. Un terrain favorable à de nouvelles concentrations?» In : DISTinfo 14 (mar. 2015), p. 8. URL : http://www.cnrs.fr/dist/z-outils/documents/Distinfo2/Distinf14.pdf. 
résultats ont perduré les années suivantes. ${ }^{10}$ On a parfois même été jusqu'à comparer l'économie de l'édition scientifique à celle de la drogue ${ }^{11}$ !

Plusieurs solutions ont été tentées pour sortir de cette situation. Dans plusieurs pays, les consortia ont négocié un accord national avec plusieurs grands éditeurs. Ces licences n'ont pas enrayé la hausse vertigineuse du coût d'accès à la documentation scientifique. Leur renégociation peut aboutir sur des ruptures, comme l'illustre l'absence de nouvellement du contrat avec Elsevier en Allemagne ${ }^{12}$ et avec Springer en France. ${ }^{13}$

Des établissements ont tenté de sortir des bouquets ${ }^{14}$, pour revenir à des abonnements titre à titre, avec des résultats décevants, tant en terme de prix que d'offre documentaire. ${ }^{15}$

\section{Un modèle économique obsolète}

Le modèle économique actuellement en vigueur dans le monde de l'édition scientifique a été conçu pour des biens rivaux. Quand une personne occupe un terrain, consomme une ressource naturelle, comme du pétrole, ou utilise un service intrinsèquement contingenté, par exemple une place dans un véhicule, elle en a l'usage exclusif et, par voie de conséquence, elle en prive les autres. Une tarification en fonction de l'utilisation, réelle ou approchée, est donc tout à fait pertinente.

La question se pose en des termes très différents lorsque qu'il s'agit d'une ressource non rivale, ce qui est la cas de la connaissance. Un célèbre passage de Thomas Jefferson, souvent cité, en faisait déjà état il y a plus de deux siècles :

Si la nature a rendu moins susceptible que toute autre chose l'appropriation exclusive, c'est bien l'action du pouvoir de la pensée que l'on appelle une idée, qu'un individu peut posséder de façon exclusive aussi longtemps qu'il la garde pour lui; mais au moment où elle est divulguée, elle devient la possession de tous, et celui qui la reçoit ne peut pas en être dépossédé. Sa propriété particulière, aussi, est que personne ne la possède moins parce que tout le monde la possède. Celui qui reçoit une idée de moi reçoit un savoir

10. EPRIST. Résultats financiers 2015 de l'édition scientifique. 30 mar. 2016. URL : http://www. eprist . fr/wp-content/uploads / 2016/03/I - IST_16_R\%C3\% A9sultatsFinanciers2015EditionSc ientifique.pdf (visité le 19/04/2018); Antoine OURY. Un chiffre d'affaires de 8,3 milliards d'euros pour RELX (Elsevier) en 2017. 16 fév. 2018. uRL : https://www .actualitte .com/article/mondeedition/un-chiffre-d-affaires-de-8-3-milliards-pour-relx-elsevier-en-2017/87344 (visité le 19/02/2018).

11. Jean-François LUTZ. "Dépenses documentaires en BU : de l'euphorie aux questionnements existentiels ». Journées RNBM - Accès ouvert, rêve ou réalité? 18 oct. 2017. URL : http://www.rnbm.org/ supports_anf/cirm2017/Presentation_LUTZ_RNBM.pdf (visité le 19/04/2018).

12. Quirin SchiERMEIER. Hundreds of German universities set to lose access to Elsevier journals. 5 déc. 2017. URL : http://www.nature.com/articles/d41586-017-07817-1 (visité le 19/02/2018).

13. Couperin ne renouvelle pas l'accord national passé avec Springer. 3 avr. 2018. URL : http: // www . couperin . org / breves / 1333 - couperin - ne-renouvelle-pas - 1-accord - national - passe avec-springer (visité le 19/04/2018); Le CNRS, Inria et une vingtaine d'établissements d'enseignement supérieur arrêtent leurs abonnements au groupe Springer. AEF info. URL : https://www. aefinfo.fr/ depeche/594873 (visité le 05/12/2018); Edition : "La licence nationale imposée à tous est inacceptable » (M. Bernard, Clermont Auvergne). Newstank education. Article 133695. 19 nov. 2018. uRL : https: / / education . newstank . fr/fr/tour/news / 133695/edition-licence-nationale-imposee-tous inacceptable-bernard-clermont.html (visité le 05/12/2018).

14. Big Deal en anglais

15. Nouvelle ère pour les collections - Bibliothèques - Université de Montréal. URL : http://www.bib. umontreal.ca/collections-nouvelle-ere/default.htm (visité le 19/04/2018). 
sans diminuer le mien; tout comme celui qui allume sa bougie à la mienne reçoit la lumière sans me plonger dans la pénombre. Que les idées circulent librement de l'un à l'autre partout sur la planète. ${ }^{16}$

Une autre caractéristique de la recherche scientifique est que son coût ne dépend pas de son nombre d'utilisateurs. Il est pour l'essentiel supporté par les institutions qui emploient les chercheurs. La publication des résultats de la recherche n'en représente qu'une faible fraction, à la fin de processus. Quant à la diffusion par voie électronique, elle a un coût très faible et, à la différence de la diffusions des revues imprimées, très peu dépendant du nombre d'utilisateurs. Par facilité de langage, on parle souvent de « coût marginal zéro », expression popularisée par Jeremy Rifkin. ${ }^{17}$

Dans ces conditions, le modèle économique de l'accès aux publications principalement fondé sur le coût marginal, par le paiement d'un abonnement, dont le tarif tient généralement compte du nombre d'utilisateurs potentiels, de la marge, toujours croissante, que souhaite réaliser l'éditeur et de la solvabilité présumée de l'institution abonnée, n'est plus légitime. Il reste cependant à trouver une solution de financement pour les coûts, essentiellement fixes, générés par le travail éditorial et la diffusion numérique. Aucun modèle ne s'est imposé pour l'instant, mais plusieurs sont en train d'émerger. C'est ce qu'on commence à appeler la « bibliodiversité ». ${ }^{18}$

\section{Un grand intérêt pour l'OA}

L'OA suscite un grand intérêt de la part de la communauté de l'ESR. Une vingtaine de déclarations ont été publiées depuis le début du $\mathrm{XXI}^{\mathrm{e}}$ siècle ${ }^{19}$ :

1. Lettre ouverte de la Public Library of Science (2001) 20

2. Initiative de Budapest pour l'Accès Ouvert (2002) ${ }^{21}$

3. Déclaration de Bethesda pour l'édition en libre accès (2003) ${ }^{22}$

4. Position de principe de l'Association of Learned and Professional Society Publishers (ALPSP, 2003) ${ }^{23}$

16. Florent LATRIVE et Lawrence Lessig. Du bon usage de la piraterie culture libre, sciences ouvertes. Essais. Paris : Exils éd., 2004. 1 vol. (170 p.) ISBN : 2-912969-59-X, pp. 32-33; Thomas Jefferson. To Isaac McPherson. 13 août 1813. URL : http://press-pubs.uchicago.edu/founders/documents/a1_8_ 8s12.html (visité le 18/04/2018).

17. Jeremy RifKin. La nouvelle société du coût marginal zéro l'internet des objets, l'émergence des communaux collaboratifs et l'éclipse du capitalisme. Trad. par Françoise CHEMLA et Paul ChemLA. 2014. 1 vol. (509 p.) ISBN : 979-10-209-0141-5.

18. Appel de Jussieu pour la Science ouverte et la bibliodiversité. 10 oct. 2017. URL : http://jussieu call.org/index-FR.html (visité le 19/04/2018).

19. La traduction française de la majorité des ces déclarations est disponible sur le site de l'INIST consacré à l'OA : Textes de références - Libre accès à l'information scientifique et technique. URL : http://openaccess.inist.fr/?-Textes-de-references- (visité le 19/02/2018).

20. PLOS Open Letter. Sept. 2001. URL : https://www.plos.org/open-letter (visité le 20/04/2018).

21. Budapest Open Access Initiative. 14 fév. 2002. URL : http://www. budapestopenaccessinitiative. org/read (visité le 20/04/2018).

22. Bethesda Statement on Open Access Publishing. 11 avr. 2003. URL : http://legacy.earlham.edu/ $\sim$ peters/fos/bethesda.htm (visité le 20/04/2018).

23. Position de principe de l'Association of Learned and Professional Society Publishers (ALPSP) Libre accès à l'information scientifique et technique. 27 août 2003. URL : http://openaccess.inist. fr/?Position-de-principe-de-1 (visité le 20/04/2018). 
5. Déclaration de Berlin sur le Libre Accès à la Connaissance en sciences exactes, sciences de la vie, sciences humaines et sociales $(2003)^{24}$

6. Déclaration de principe du Wellcome Trust en soutien à l'édition en Libre Accès $(2003)^{25}$

7. Position de l'InterAcademy Panel sur l'accès à l'information scientifique (2003) ${ }^{26}$

8. Déclaration de l'IFLA sur le libre accès à la littérature scientifique et aux documents de la recherche $(2003)^{27}$

9. Déclaration de Valparaiso pour une meilleure communication scientifique sur support électronique (2004) ${ }^{28}$

10. Déclaration de l'OCDE sur l'accès aux données de la recherche financée par des fonds publics (2004) 29

11. Principes de Washington D.C. pour le libre accès à la science (2004) ${ }^{30}$

12. Déclaration écossaise sur le Libre Accès (2004) ${ }^{31}$

13. Déclaration d'EBLIDA (2005) ${ }^{32}$

14. Déclaration de Salvador sur le Libre Accès (2005) ${ }^{33}$

15. Nidae ar-Ryadh / Appel de Riyad (2006) ${ }^{34}$

16. Déclaration sur le Libre Accès du Conseil scientifique du CER (2006) ${ }^{35}$

17. Déclaration de l'Alhambra sur le Libre Accès (2010) ${ }^{36}$

24. Berlin Declaration. 22 oct. 2003. URL : https://openaccess.mpg.de/Berlin-Declaration (visité le $20 / 04 / 2018$ ).

25. Une déclaration de principe du Wellcome Trust en soutien à l'édition en Libre Accès - Libre accès à l'information scientifique et technique. Nov. 2003. URL : http : // openaccess . inist . fr / ?Unedeclaration-de-principe-du (visité le 20/04/2018).

26. IAP. IAP Statement on Access to Scientific Information. 4 déc. 2003. URL : http://interacadem ies.org/13916/IAP-Statement-on-Access-to-Scientific-Information (visité le 20/04/2018).

27. Déclaration de l'IFLA sur le libre accès à la littérature scientifique et aux documents de la recherche - Libre accès à l'information scientifique et technique. 5 déc. 2003. URL : http://openaccess. inist. fr/?Declaration-de-1-IFLA-sur-le-libre (visité le 20/04/2018).

28. Déclaration de Valparaiso pour une meilleure communication scientifique sur support électronique - Libre accès à l'information scientifique et technique. 15 jan. 2004. URL : http://openaccess. inist. fr/?Declaration-de-Valparaiso-pour-une (visité le 20/04/2018).

29. Science, Technology and Innovation for the 21st Century. Meeting of the OECD Committee for Scientific and Technological Policy at Ministerial Level, 29-30 January 2004 - Final Communique - OECD. 30 jan. 2004. URL : http : / / www . oecd . org / science / sci tech / sciencetechnologyandinnovationforthe21stcenturymeetingof theoecdcommitteeforscientif icandtechnologicalpolicyatministeriallevel29-30january2004-finalcommunique . htm (visité le 20/04/2018).

30. Washington D.C. Principles For Free Access to Science : A Statement from Not-for-Profit Publishers. 16 mar. 2004. URL : http://www.dcprinciples.org/statement.pdf (visité le 20/04/2018).

31. Déclaration écossaise sur le Libre Accès - Libre accès à l'information scientifique et technique. 11 oct. 2004. URL : http://openaccess.inist.fr/?Declaration-ecossaise-sur-le-Libre (visité le 20/04/2018).

32. Déclaration d'EBLIDA - Libre accès à l'information scientifique et technique. Avr. 2005. URL : http://openaccess.inist.fr/?Declaration-d-EBLIDA (visité le 20/04/2018).

33. Salvador Declaration on Open Access : the developing world perspective. 23 sept. 2005. URL : http: //www.icml.org/public/documents/pdf/en/Dcl-Salvador-OpenAccess-en.pdf (visité le 20/04/2018).

34. Nidae ar-Ryadh / Appel de Ryadh - Libre accès à l'information scientifique et technique. Fév. 2006.

URL : http://openaccess.inist.fr/?Nidae-ar-Ryadh-Appel-de-Ryadh (visité le 20/04/2018).

35. «ERC Scientific Council Statement on Open Access ». In : (déc. 2006), p. 1.

36. Déclaration de l'Alhambra sur le Libre Accès - Libre accès à l'information scientifique et technique. 14 mai 2010. URL : http://openaccess.inist.fr/?Declaration-de-1-Alhambra-sur-le (visité le 20/04/2018). 
18. Pour un meilleur accès aux informations scientifiques : dynamiser les avantages des investissements publics dans le domaine de la recherche (Déclaration de Commission européenne, 2012) ${ }^{37}$

19. Déclaration de Dakar sur la publication en Open Access en Afrique et dans les pays du Sud (2016) ${ }^{38}$

20. Appel de Jussieu pour la science ouverte et la bibliodiversité (2017) ${ }^{39}$

21. Déclaration de Delhi sur l'Open Access (2018) ${ }^{40}$

22. Déclaration de Mexico en faveur de l'écosystème latino-américain de l'Open Access non commercial $(2018)^{41}$

23. Déclaration de Thessalonique de soutien au libre accès et d'engagement à accélérer la transition du Southern European Libraries Link (SELL) (2018) ${ }^{42}$

Au-delà de ces déclarations solennelles, d'excellentes et courtes vidéos pédagogiques ont été réalisées pour expliquer comment le modèle économique actuel ne peut plus durer et pourquoi l'OA est la solution. ${ }^{43}$

Mais les actes sont-ils à la hauteur de toutes ces intentions? La majorité des déclarations est publiée entre 2001 et 2006. La déclaration fondatrice est l'Initiative de Budapest, en 2002. Les autres sont essentiellement des déclarations d'adhésion et d'adaptation locale ou sectoriellle. La reprise des déclarations, à partir de 2010, peut laisser penser que les précédentes ont peu été suivies d'effets. L'objet du présent rapport est justement de tenter de savoir où en est la longue route de l'OA et de proposer des pistes pour la poursuivre.

Le chapitre 1 montrera comment l'OA s'est complexifié, bien au-delà des deux voies verte et dorée originale, parfois à la limite ou en dehors de la légalité. Quelques exemples de politiques nationales seront exposés.

Le chapitre 2 tentera un état des lieux de l'OA dans le monde de l'ESR français. On y abordera les archives ouvertes, dominées par HAL, les initiatives académiques en faveur de l'OA et les manifestations pour sa promotion. Les données recueillies à cette occasion figureront en annexe.

Puis le chapitre 3 traitera, sous forme d'une FAQ, des obstacles fréquemment opposés au développement de l'OA.

37. COMMISSION EuRopéEnNe. Pour un meilleur accès aux informations scientifiques : dynamiser les avantages des investissements publics dans le domaine de la recherche. 17 juil. 2012. URL : http: //ec . europa.eu/research/science-society/document_library/pdf_06/era-communicationtowards-better-access-to-scientific-information_fr.pdf (visité le 19/04/2018).

38. Dakar Declaration on Open Access Publishing in Africa and the Global South / CODESRIA. $1^{\mathrm{er}}$ avr. 2016. URL : http://www.codesria.org/spip.php?article2595 (visité le 27/04/2018).

39. Appel de Jussieu pour la Science ouverte et la bibliodiversité, op. cit.

40. Delhi Declaration on Open Access. 14 fév. 2018. URL : http://openaccessindia.org/delhideclaration-on-open-access/ (visité le 24/04/2018).

41. Declaration de Mexico : A favor del Ecosistema latinoamericano de Acceso Abierto No Comercial. URL : http://www.accesoabiertoalyc.org/declaracion-mexico/ (visité le 19/02/2018).

42. SELL signe une déclaration commune concernant l'open access. 5 juin 2018. URL : https://www . couperin.org/breves/1342-le-sell-signe-une-declaration-commune-concernant-1-open-access (visité le 06/09/2018).

43. Data Gueule. Privés de savoir? \#DATAGUEULE 63. 17 oct. 2016. URL : https://www . youtube . com/watch?v=WnxqoP-c0ZE (visité le 19/02/2018); Open Access Explained! URL : http://phdcomics . com/tv/?v=L5rVH1KGBCY (visité le 19/02/2018). 
Enfin, le chapitre 4 fera une série de propositions pour avancer, dans les domaines de la communication sur le droit, de l'évaluation des chercheurs, du rôle des établissements d'ESR et de leurs services communs de documentation (SCD) et de HAL. Il finira en proposant la création d'un outil de mutualisation. 


\section{Chapitre 1}

\section{Open Access : de quoi parle-t-on?}

Pendant longtemps, on a pu se contenter de parler des deux voies de l'Open Access (OA), la voie verte, ou Green $O A$ et la voie dorée, ou Gold OA, définies en 2002 dans l'Initiative de Budapest. Ces deux voies ont été théorisées par Peter Suber. ${ }^{1}$ Pourtant, au fil du temps, le paysage s'est fortement complexifié ${ }^{2}{ }^{3}$

\subsection{Green OA : la voie verte}

\subsubsection{Archives ouvertes}

La voie verte est ancienne. Elle repose sur l'auto-archivage par les chercheurs de leurs propres publications, sur des serveurs contrôlés par leur communauté. L'élément de base de la voie verte est l'article. L'expression Archive ouverte (AO), au sens propre, désigne ce type de dépôt. L'exemple le plus ancien semble être arXiv, fondé par Paul Ginsparg dès 1991. A l'origine, arXiv était destiné au dépôt des preprints en physique. Le dépôt a ensuite été étendu aux mathématiques, à l'informatique, au génie électrique, à la biologie et à la finance quantitatives, aux statistiques et à l'économie. ${ }^{4}$

En France, le principal dépôt d'archives ouvertes est HAL. ${ }^{5}$ HAL signifie Hyper articles en ligne, mais est toujours désigné par son seul acronyme. HAL a été créé en 2001 par le Centre pour la Communication Scientifique Directe (CCSD) du CNRS. ${ }^{6}$ Outre les preprints, HAL accepte également les postprints, les thèses et HDR, les livres

1. Peter Suber. Open Access Overview : Focusing on open access to peer-reviewed research articles and their preprints. 21 juin 2004. URL : http://legacy.earlham.edu/ peters/fos/overview.htm (visité le 19/02/2018).

2. Pour compléter sur l'histoire de l'OA, on pourra consulter : Annaig MAHÉ. Bibliothèques et archives ouvertes. $1^{\text {er }}$ jan. 2011. URL : http://bbf .enssib.fr/consulter/bbf-2011-01-0014-003 (visité le 16/04/2018); Ghislaine Chartron. « Open Access : origine, enjeux, actualités ». 11 fév. 2013. URL : http : / / ww . openaccess - shs . info / wp-content / uploads / 2012 / 12 / Pr \% C3\% A9sentation-deGhislaine-Chartron-11-f\%C3\%A9vrier .pdf (visité le 19/04/2018); Peter SuBER. History of open access. URL : https : / / cyber . harvard . edu/ psuber / wiki / History _ of _ open_access (visité le $24 / 04 / 2018)$.

3. Le portail global de l'Unesco regroupe de nombreuses informations sur l'OA issues du monde entier, mais sa mise à jour semble arrêtée depuis 2015 : Global Open Access Portal (GOAP). United Nations Educational, Scientific and Cultural Organization. URL : http: //www . unesco.org/new/en/ communication-and-information/portals-and-platforms/goap/ (visité le 19/04/2018).

4. arXiv.org e-Print archive. URL : https://arxiv.org/ (visité le 19/02/2018).

5. Archive ouverte HAL. URL : https://hal archives-ouvertes.fr/.

6. CCSD. Les temps forts. URL : https://www.ccsd.cnrs.fr/presentation/les-temps-forts/ (visité le 19/02/2018). 
et leurs chapitres, les communications dans un congrès et les posters, et plusieurs autres types de publications, dans toutes les disciplines, ainsi que le code source de logiciels. ${ }^{7}$

\subsubsection{Preprints et postprints}

La notion de preprint ${ }^{8}$, ne fait guère débat. Il s'agit du texte proposé par un chercheur avant sa lecture critique ${ }^{9}$ par un comité de pairs de sa discipline ${ }^{10}$. Ces derniers peuvent faire des demandes de correction, auxquelles le chercheur répond, au cours d'un processus itératif qui pourra, de fait, générer plusieurs versions du même preprint.

Au contraire, la notion de postprint peut désigner des réalités assez diverses. Il peut s'agit de la « version auteur », au sens strict ${ }^{11}$, c'est-à-dire du dernier document envoyé par l'auteur à l'éditeur, incluant toutes les corrections demandées par le comité de lecture, mais sans la mise en page de l'éditeur (notamment la pagination dans la revue), et, s'ils sont réalisés par l'éditeur, sans les graphiques et autres illustrations. De cette acception, le postprint est un document moins complet que la version intégrale de l'article publié publiée dans une revue, dite version éditeur. On constate cependant assez souvent l'existence de faux post-prints, réalisés à partir de la version publiée par l'éditeur $^{12}$, dans laquelle certaines informations (logo de l'éditeur, numérotation des pages, titre courant...) ont été volontairement masqués. On peut considérer que cette manipulation est une perte de temps absurde, qui consiste à dégrader la qualité d'un document pour lui conférer un statut fictif de post-print auteur.

Les politiques des éditeurs à l'égard du dépôt en archive ouverte sont très variables. Certains n'autorisent aucun dépôt, d'autres seulement le preprint. Ces politiques contreviennent désormais à l'article 30 de la loi 2016-1321 lorsqu'il s'agit du résultat de recherches financées au moins pour moitié par des fonds publics. D'autres éditeurs autorisent encore le dépôt du postprint et parfois même la version éditeur, avec un embargo le cas échéant. De trop nombreux éditeurs n'ont tout simplement pas de politique formalisée. ${ }^{13}$

\subsubsection{Archive ouverte thématique, nationale ou institutionnelle?}

Sur le modèle d'arXiv, d'autres archives ouvertes thématiques ont été créées plus récemment autour du Center for Open Science ${ }^{14}$ et de son Open Science Framework ${ }^{15}$ :

7. Agnès Magron. Le dépôt de logiciel maintenant disponible sur HAL et tous les portails / CCSD. 25 sept. 2018. URL : https : //www . ccsd. cnrs .fr/2018/09/le-depot-de-logiciel-maintenantdisponible-sur-hal-et-tous-les-portails/ (visité le 05/12/2018).

8. ou prépublication ou version soumise

9. peer review en anglais

10. reviewers ou referees en anglais

11. ou postdocument, postprint auteur, version finale acceptée pour publication (terme employé dans l'article 30 de la Loi pour une République numérique), manuscrit accepté, accepted manuscript, final draft post-reffering...

12. ou postprint éditeur, publisher's version, PDF éditeur...

13. On trouvera d'utiles explications complémentaires sur la page des questions juridiques de la documentation de HAL : Questions juridiques - HAL Documentation. URL : https ://doc.archivesouvertes.fr/questions-juridiques/ (visité le 20/02/2018).

14. The Center for Open Science. URL : https://cos.io/ (visité le 23/04/2018).

15. OSF Preprints. URL : https://osf.io/preprints/ (visité le 23/04/2018). 
AgriXiv $^{16}$ (agriculture), BioRXiv ${ }^{17}$ (biologie), ChemArXiv ${ }^{18}$ (chimie), EarthArXiv ${ }^{19}$ (sciences de la terre), engrXiv ${ }^{20}$ (ingénierie), LawArxiv ${ }^{21}$ (droit), LISSA ${ }^{22}$ (sciences de l'information et des bibliothèques), MarXiv ${ }^{23}$ (océanographie), MindRxiv ${ }^{24}$ (recherche sur les méditation et les pratiques contemplatives), NutriXiv ${ }^{25}$ (sciences de la nutrition), PaleorXiv ${ }^{26}$ (paléontologie), PsyArXiv ${ }^{27}$ (psychologie), SocarXiv ${ }^{28}$ (science sociales), et SportRxiv ${ }^{29}$ (sport et activité physiques).

PubMed Central ${ }^{30}$ est une archive ouverte en sciences biomédicale et de la vie, créée en 2000 à l'initiative du National Institutes of Health's National Library of Medicine (NIH/NLM), agence gouvernementale des Etats-Unis.

Les économistes ont créé leur propre éco-système d'archives ouvertes et de services associés avec RePEc (Research Papers in Economics). ${ }^{31}$

HAL représente un cas unique au monde d'archive ouverte nationale pluridisciplinaire. ${ }^{32}$ Cependant, dans les disciplines concernées, un document déposé dans HAL peut être automatiquement et simultanément versé dans arXiv.

Certains établissements d'ESR ont fait le choix d'avoir leur propre archive ouverte institutionnelle (AOI). La démarche dépasse celle des archives ouvertes thématiques ou pluridisciplinaires, car elle est à l'initiative de l'établissement employant le chercheur. De plus, outre la diffusion des résultats de la recherche, elle a pour objectif de rendre visible la production scientifique de l'établissement et éventuellement, de produire des outils bibliométriques.

Bien qu'il s'agisse d'abord d'une archive nationale pluridisciplinaire, HAL propose aux établissements qui le souhaitent de créer des portails, qui, de fait, tiennent lieu d'archive ouverte institutionnelle. ${ }^{33}$ Cette solution est celle majoritairement employée par les établissements d'ESR et les organismes de recherche français. Il existe également quelques portails disciplinaires des HAL. Il faut cependant garder à l'esprit que HAL est un dépôt unique et que les portails ne sont que des vues partielles sur ce dépôt. Si les métadonnées sont correctement renseignées, un document déposé dans HAL apparâ̂-

16. Agrixiv. URL : https://agrixiv.org/ (visité le 23/04/2018).

17. bioRxiv.org - the preprint server for Biology. URL : https : //www . biorxiv . org/ (visité le 20/02/2018).

18. ChemArxiv Repository. URL : http://chemarxiv.org/ (visité le 20/02/2018).

19. eartharxiv. URL : https://eartharxiv.org/ (visité le 23/04/2018).

20. engrXiv. URL : https://engrxiv.org/ (visité le 23/04/2018).

21. LawArXiv. URL : https://osf .io/preprints/lawarxiv (visité le 23/04/2018).

22. LISSA : Library and information science Scholarship Archive Preprints. URL : https://osf.io/ preprints/lissa (visité le 23/04/2018).

23. MarXiv. URL : https://marxiv.org/ (visité le 23/04/2018).

24. MindRxiv. URL : https://www.mindandlife.org/mindrxiv/ (visité le 23/04/2018).

25. NutriXiv. URL : https://osf .io/preprints/nutrixiv (visité le 23/04/2018).

26. PaleorXiv. URL : https://paleorxiv.org/ (visité le 23/04/2018).

27. PsyArXiv preprints. URL : https://psyarxiv.com/ (visité le 20/02/2018).

28. SocArXiv papers : Open archive of the social sciences. URL : https://osf .io/preprints/socarxiv (visité le 20/02/2018).

29. SportRxiv. URL : https://osf.io/preprints/sportrxiv (visité le 23/04/2018).

30. PubMed Central (PMC). URL : https://www.ncbi.nlm.nih.gov/pmc/ (visité le 16/04/2018).

31. RePEc : Research Papers in Economics. URL : http://repec.org/ (visité le 16/04/2018); L'archive ouverte proprement dite s'appelle EconPaper : EconPapers. URL : https : //econpapers.repec .org/ (visité le 23/04/2018).

32. Une recherche sur OpenDOAR ne fait pas apparaître d'autres pays dans ce cas : OpenDOAR : Directory of Open Access Repositories. URL : http://www.opendoar.org/ (visité le 17/02/2018).

33. HAL : Les portails de l'archive. URL : https ://hal .archives-ouvertes .fr/browse/portal (visité le 17/01/2018). 
tra donc automatiquement dans le portail HAL de l'établissement de rattachement du chercheur et réciproquement.

\subsubsection{La voie verte dans le monde}

Le Directory of Open Access Repositories (OpenDOAR) est un service de SHERPA, qui a pour objectif de recenser tous les dépôts d'archives ouvertes existant au monde. ${ }^{34}$ Le 20 février 2018, il identifiait 3503 dépôts d'archives ouvertes, dont 46\% en Europe, $20 \%$ en Asie et $18 \%$ en Amérique du Nord. $86 \%$ des dépôts d'archives ouvertes sont institutionnels et $12 \%$ sont thématiques. ${ }^{35}$

La Confederation of Open Access Repositories (COAR) est une association internationale de plus de 140 membres. ${ }^{36}$ Elle porte en particulier le projet Next Generation Repositories, qui a pour ambition de relier tous les dépôts d'archive ouverte pour en faire un réseau mondial distribué d'accès et de services autour des ressources en OA, piloté collectivement par la communauté de la recherche. ${ }^{37}$

$C O R E$ est un projet qui fédère des partenaires publics et privés, afin de fournir une interface de recherche mondiale des articles en OA. ${ }^{38}$

Faute d'une vue d'ensemble sur les politiques nationales en faveur de la voie verte, on pourra s'attarder sur quelques cas.

\section{Belgique}

L'exemple qui a le plus marqué les esprits français est celui de l'Université de Liège, en Belgique. Son ancien recteur, Bernard Rentier, est à l'origine d'une archive ouverte institutionnelle, d'abord nommée Digithèque ULg, puis connue sous le nom d'ORBI (Open Repository and Bibliography), ${ }^{39}$ ouverte en 2007. Dès son origine, cette archive ouverte a été assortie d'une obligation de dépôt de tous les articles publiés par les membres de l'université, sous forme du postprint éditeur. ${ }^{40}$ De plus, les différents comités d'évaluation de l'université ne doivent prendre en compte dans tout processus d'évaluation que les publications déposées dans ORBI. ${ }^{41}$ Petite subtilité, si le dépôt du texte intégral est obligatoire, l'auteur conserve le droit de refuser sa publication en OA.

L'Université catholique de Louvain, en partenariat avec l'Université de Namur et l'Université Saint-Louis de Bruxelles, a mis en place un dispositif similaire en 2013, nommé DIAL. ${ }^{42}$ L'Université libre de Bruxelles a également sa propre archive ouverte institutionnelle, Di-fusion, ${ }^{43}$ mais n'a pas rendu obligatoire le dépôt des publications de

34. OpenDOAR : About. URL : http://www . opendoar .org/about.html (visité le 20/02/2018); Il existe aussi le Registry of Open Access Repositories. URL : http://roar.eprints.org/ (visité le 16/04/2018).

35. OpenDOAR : Charts - Worldwide. URL : http://www . opendoar.org/find.php? format=charts (visité le 20/02/2018).

36. Confederation of Open Access Repositories (COAR) - Towards a global knowledge commons. URL : https://www. coar-repositories.org/ (visité le 31/05/2018).

37. COAR Next Generation Repositories : Vision and Objectives. URL : http://ngr.coar-repositor ies.org/ (visité le 06/06/2018).

38. CORE. URL : https://core.ac.uk/ (visité le 06/06/2018).

39. ORBi. URL : https://orbi.uliege.be/ (visité le 20/02/2018).

40. ORBi : Contenu. URL : https://orbi.uliege.be/project?id=103 (visité le 20/02/2018).

41. Université de LièGE. Extrait du procès-verbal de la séance du 23 mai 2007 du Conseil d'administration. URL : https://orbi.uliege.be/files/extrait_moniteur_CA.pdf (visité le 20/02/2018).

42. DIAL. URL : https://dial.uclouvain.be/Home/ (visité le 20/02/2018).

43. DI-fusion. URL : http://difusion.ulb.ac.be/ (visité le 20/02/2018). 
ses chercheurs. Cependant, depuis 2013, seules les publications signalées dans Di-fusion sont prise en compte lors des opérations d'évaluation. ${ }^{44}$

Le 2 mai 2018, le parlement de la Fédération Wallonie-Bruxelles a adopté, à la quasiunanimité $^{45}$, le Projet de décret 603 (2017-2018) visant à l'établissement d'une politique de libre accès aux publications scientifiques (Open Access). ${ }^{46}$ Ce texte généralise le modèle liégeois à toute la Belgique francophone. Il a fait l'objet de campagnes de la la part de ses promoteurs ${ }^{47}$ et de ses détracteurs. ${ }^{48}$

\section{Luxembourg}

Suivant le modèle de l'Université de Liège, et avec son aide, l'Université du Luxembourg a créé sa propre archive ouverte institutionnelle, ORBi ${ }^{l u}$, assortie d'un mandat d'accès ouvert pour tous les articles et présentations publiés à compter du $1^{\mathrm{er}}$ janvier 2009. ${ }^{49}$

\section{Espagne}

L'Espagne semble être un des rares pays à avoir fait de la voie verte une politique nationale. L'article 37 de la loi pour la science de $2011^{50}$ rend obligatoire la publication des articles scientifiques issus d'une recherche réalisée avec des fonds publics dans des dépôts thématiques ou institutionnels, le plus tôt possible et au plus tard douze mois après la date de publication. Cependant, deux limites importantes restreignent les effets de ce texte : les politiques des éditeurs ont priorité sur la loi et aucune mesure n'a été prévue pour s'assurer du respect de l'article 37. De ce fait, bien que la quasi totalité des universités espagnoles aient créé leur propre AO, moins de $10 \%$ des publications relevant de l'article 37 y ont été déposées. ${ }^{51}$

44. DI-fusion: Guide du déposant. URL : https://www.bib.ulb.ac.be/fr/bibliotheque-electroni que/depot-institutionnel-di-fusion/guide-du-deposant/index.html (visité le 20/02/2018).

45. 76 voix pour, 0 contre, 2 abstentions

46. Projet de décret 603 (2017-2018) visant à l'établissement d'une politique de libre accès aux publications scientifiques (Open Access). 7 mar. 2018. URL : http://archive.pfwb.be/1000000020870ee; Projet de décret visant à l'établissement d'une politique de libre accès aux publications scientifiques (Open Access) - Parlement de la Fédération Wallonie-Bruxelles / Communauté française. 7 mar. 2018. URL : http : / www . pfwb . be / le-travail-du-parlement/doc-et-pub/documents-parlementaires-etdecrets/dossiers/001591645 (visité le 23/04/2018).

47. A. Louvigny. Publications scientifiques en accès libre: la mentalité des chercheurs doit aussi évoluer. 19 mar. 2018. URL : https://www.rtbf.be/info/societe/detail_publications-scientifi ques-en-acces-libre-la-mentalite-des-chercheurs-doit-aussi-evoluer?id=9871133 (visité le $29 / 03 / 2018)$.

48. ADEB. OPEN ACCESS : Un projet de décret sans concertation des parties prenantes menace l'édition scientifique. $1^{\mathrm{er}}$ mar. 2018. URL : http://adeb.be/sites/502a034d9f83256166000004/assets/ 5a9954031dd64768af000964/Communiqu__de_presse_-_0A.pdf (visité le 23/04/2018).

49. Mandat de dépôt. URL : http://orbilu.uni.lu/project?locale=fr\&id=project-mandate (visité le $03 / 04 / 2018$ ).

50. Ley 14/2011, de 1 de junio, de la Ciencia, la Tecnología y la Innovación. $1^{\text {er }}$ juin 2011. URL : http://www. boe.es/buscar/pdf/2011/BOE-A-2011-9617-consolidado.pdf (visité le 19/02/2018), Artículo 37. Difusión en acceso abierto.

51. Lluis ANGLADA. "L'accès ouvert en Espagne : mandat, état des lieux et approche progressive actuelle». JSO 2018. 23 jan. 2018. URL : https://jso2018. sciencesconf.org/data/pages/Lluis_ Anglada_2.pdf (visité le 19/02/2018). 


\section{Argentine}

L'Argentine a adopté en 2013 une loi proche de la loi espagnole. ${ }^{52}$

\section{France}

En France, la voie verte ne peut pas être considérée comme une politique nationale, mais elle y bénéficie de conditions favorables. D'une part, la France est le seul pays à avoir développé une plateforme nationale d'archives ouvertes, HAL, au sein de laquelle les établissements peuvent avoir leur propre portail institutionnel. Cette infrastructure de recherche, reconnue comme telle par le Ministère de l'Enseignement Supérieur et de la Recherche (MESR) en 2016, simplifie fortement la tâche technique des établissements d'ESR. Depuis 2016, les utilisateurs de HAL se sont organisés en club, sous la forme de l'assocation CasuHAL. ${ }^{53}$ D'autre part, la loi Lemaire, retranscrite dans le Code de la recherche (article L533-4), ${ }^{54}$ autorise les chercheurs dont l'activité est financée au moins pour moitié sur fonds publics a déposer leur postprint dans une archive ouverte, au plus tard un an après la publication de l'article par l'éditeur.

\section{Pays-Bas}

Bien que fortement engagés dans la voie dorée, comme détaillé dans la section suivante du présent chapitre, les Pays-Bas ont fédéré leurs dépôts d'archives ouvertes au sein du portail NARCIS. ${ }^{55}$

\subsection{Gold OA : la voie dorée}

\subsubsection{Auteur-payeur et APC}

A l'origine, la voie dorée ne définissait pas un modèle économique, mais seulement les revues à comité de lecture immédiatement et gratuitement accessibles en ligne. Le Directory of Open Access Journals (DOAJ) recensait au printemps 2018 plus de 11000 revues répondant à cette définition, comprenant plus de trois millons d'articles et éditées dans 126 pays. ${ }^{56}$

Dans la voie dorée, l'élément de base est la revue et non l'article. De ce fait, l'initiative de la voie dorée revient à l'éditeur, qu'il soit commercial, public ou coopératif, et non au chercheur. Une revue à comité de lecture a forcément un coût de production plus élevé qu'un dépôt d'archive ouverte. Dans les faits, la voie dorée repose très fréquemment sur le paiement par l'auteur, ou, le plus souvent, par son institution, de frais de publications à l'éditeur, qui, en échange, publie l'article en libre accès immédiat.

Les frais des publication sont habituellement connus sous leur nom anglais d'Article Processing Charge, presque toujours désignés par le sigle APC. Comme leur nom l'indique, ils sont censés couvrir les frais de publication, mais de fait, il varient selon le

52. Sistema Nacional de Repositorios Digitales - República Argentina. URL : http://repositorios .min cyt.gob.ar/recursos.php (visité le 25/04/2018); Sistema nacional de ciencia, tecnologia e innovacion. 3 déc. 2013. URL : http://repositorios.mincyt.gob.ar/pdfs/Boletin_Oficial_Ley_26899.pdf (visité le 25/04/2018).

53. CasuHAL : club utilisateur. URL : https://iww.inria.fr/casuhal/ (visité le 24/04/2018).

54. Code de la recherche - Article L533-4. URL : https://www.legifrance.gouv.fr/affichCodeArti cle.do?idArticle=LEGIARTI000033205794\&cidTexte=LEGITEXT000006071190 (visité le 20/02/2018).

55. NARCIS. URL : https://www.narcis.nl/ (visité le 23/04/2018).

56. Directory of Open Access Journals. URL : https://doaj.org (visité le 30/04/2018). 
prestige de la revue. Rarement inférieurs à $1000 €$, somme qui semble correspondre aux coûts réels, ils peuvent dépasser les $5000 €$, la moyenne se situant un peu au dessous de $2000 €{ }^{57}$

A côté des revues entièrement en Gold OA, il existe des revues dites hybrides, diffusées sur abonnement payant, mais dans lesquelles il est possible de de publier des articles en Gold OA. ${ }^{58}$ Le modèle des revues hybrides fait l'objet de vives critiques, car, au lieu de faire baisser le coût de l'accès aux articles scientifiques pour les institutions, il l'augmente, l'institution continuant à payer les coût de l'abonnement, auquel s'ajoute celui des APC pour les articles qu'elle a choisi de publier en OA.

\subsubsection{Bronze OA : la forme dégradée du Gold}

Bien que peu employé, le concept de Bronze OA est apparu en 2018 pour désigner des publications librement accessibles sur le site de leur éditeur, mais sans qu'une licence libre, permettant leur réutilisation, ne leur soit attribuée. D'après les inventeurs du concept, cette forme d'OA serait la plus répandue. On peut considérer le Bronze $O A$ comme une forme dégradée du Gold $O A$, dont peu d'acteurs ont pour l'instant conscience. ${ }^{59}$

\subsubsection{La transition dorée vers l'OA?}

Certains pays européens ont fait le choix de la transition dorée vers l'OA, généralement par le biais de contrat globaux ${ }^{60}$ passés avec les principaux éditeurs, incluant à la fois un abonnement aux bouquets pour leurs établissements d'ESR et le paiement d'APC pour la publication en libre accès des articles de leurs chercheurs.

Cette transition vers la voie dorée fait l'objet de contestation de la part de chercheurs, notamment dans le domaine des mathématiques. ${ }^{61}$ D'abord au niveau économique : le total des APC sera-t-il moins élevé que les abonnements qu'ils remplacent, autant, ou plus? Et plus encore au niveau des enjeux de pouvoir : qui doit contrôler l'édition, la communauté scientifique ou les éditeurs commerciaux? Les chercheurs craignent que la transition dorée renforce en fait la position dominante de l'oligopole de l'édition scientifiques pour de nombreuses années. ${ }^{62}$

Sans prétendre à l'exhaustivité, quelques politiques nationales sont présentées ciaprès.

57. OpenAPC : APC paid by publishers. URL : https ://treemaps . intact-project.org/apcdata/ openapc/\#publisher/ (visité le 20/02/2018).

58. Hybrid open access journal. In : Wikipedia. Page Version ID : 817351083. 27 déc. 2017. URL : https : //en . wikipedia.org/w/index .php?title=Hybrid_open_access_journal\&oldid=817351083 (visité le 20/02/2018).

59. Heather PIWOWAR et al. « The state of OA : a large-scale analysis of the prevalence and impact of Open Access articles ». In : PeerJ 6 (13 fév. 2018), e4375. ISSN : 2167-8359. DOI : 10.7717/peerj .4375. URL : https://peerj.com/articles/4375 (visité le 05/12/2018).

60. offset agreements ou compact agreements en anglais

61. Conseil scientifique de l'institut des mathématiques (INSMI) CNRS. Recommandation au sujet des frais de publication (APC). 15 juin 2016. URL : http : / www . cnrs.fr/comitenational/doc/ recommandations/2016/Recommandation-csi-INSMI-au-sujet-des-frais-de-publication-(APC) .pdf (visité le 08/03/2018).

62. MARIE FARge et Frédéric HÉLein. Transition vers l'Accès Libre : le piège des accords globaux avec les éditeurs. 13 avr. 2018. URL : https ://blogs . mediapart.fr/edition/au-coeur-de-larecherche/article/130418/transition-vers-l-acces-libre-le-piege-des-accords-globauxavec-les-editeur (visité le 24/04/2018). 


\section{Royaume-Uni}

Le Royaume-Uni s'est engagée dans cette voie dès 2012, sur la base du Rapport Finch ${ }^{63}$ :

Ce rapport soulignait entre autres que l'édition scientifique britannique était une industrie forte, créatrice d'emplois et générant d'importants surplus commerciaux. Dans ce contexte le Gold Open Access porté par les éditeurs scientifiques eux-mêmes, apparaissait comme le seul modèle économique permettant une transition vers le libre accès à la publication scientifique sans fragiliser cette industrie. ${ }^{64}$

A partir de 2014, le JISC, qui assure notamment les fonction de consortium pour le Royaume-Uni, a entrepris des négociations avec les éditeurs pour transformer progressivement des budgets d'abonnement en budgets d'APC. Ces nouveaux contrats sont appelés « offset agreements », habituellement traduit pas « accords couplés ».

Un rapport du JISC, publié en octobre 2016, a analysé les cinq premiers contrats signés avec de grand éditeurs ${ }^{65}$. Il apparaît que ces accords sont complexes, avec de très nombreux paramètres, et différents d'un éditeur à l'autre. Seules les institutions ayant souscrit un «big deal » bénéficient de réduction sur leurs APC. Plus grave, les APC ne viennent pas en déduction du prix des abonnements, mais font simplement l'objet d'un tarif réduit pour les institutions abonnées. L'essentiel des APC est payé à des revues hybrides. Cette transition vers le Gold OA a donc permis d'augmenter le nombre d'articles publiés en $\mathrm{OA}$, mais pas de faire baisser ni même de stabiliser les dépenses documentaires scientifiques, les APC ayant été payés par des agences de financement de la recherche. ${ }^{66}$

\section{Pays-Bas}

Les Pays-Bas, dont l'association néerlandaise des universités (VSNU) sert de consortium, ont négocié avec les principaux éditeurs pour aboutir à $100 \%$ de Gold OA, pour un coût moins élevé que les anciens abonnements. Lors des Journées Science Ouverte 2018, Koen Becking, président de l'Université de Tilburg et responsable des négociations pour l'open access à VSNU, a déclaré "we don't want dinners and talks, just OA for less money". Les huit plus gros éditeurs représentent $70 \%$ du marché, soit 7,6 MM $€$ d'où un APC moyen de $3800^{\circ} €$. L'objectif est de parvenir à un coût total de $4 \dot{M M M}^{\circ} €$, soit un APC moyen de $2000^{\circ} €$. En 2018, $57 \%$ de l'offre des sept plus gros éditeurs étaient

63. Janet FinCH. Accessibility, sustainability, excellence : how to expand access to research publications. Juin 2012. URL : https : //www . acu . ac.uk/research-information-network/finch-report-final (visité le 20/02/2018).

64. Michel VAJOU. « Une étude britannique met en lumière les effets secondaires négatifs des « big deals » associant négociation des budgets d'abonnements et des budgets d'APC ». In : EPRIST Analyse I/IST 24 (nov. 2016). URL : http://www.eprist.fr/wp-content/uploads/2016/11/I-IST_24EtudeJISC.pdf (visité le 20/02/2018).

65. Institute of Physics, Royal Society of Chemistry, Taylor \& Francis, Sage et Wiley

66. VAJOU, «Une étude britannique met en lumière les effets secondaires négatifs des « big deals » associant négociation des budgets d'abonnements et des budgets d'APC », op. cit.; Katie SHAmASH. Article processing charges (APCs) and subscriptions : Monitoring open access costs. 27 juin 2016. URL : https://www.jisc.ac.uk/reports/apcs-and-subscriptions (visité le 20/02/2018). 
en Gold OA, alors que les objectifs du gouvernement, qui appuie cette voie négociée, n'étaient que de $30 \%$. ${ }^{67}$

\begin{abstract}
Allemagne
En Allemagne, la Société Max-Planck a lancé l'Open Access 2020 Alliance (OA2020). Malgré de nombreuses déclarations de principe en faveur de l'OA, le Green OA ne représente que $15 \%$ de la production scientifique. l'OA2020 promeut en conséquence le PAR (Publish And Read) model, qui consiste à remplacer les abonnements par des APC à un coût transparent et soutenable, pour une publication généralisée en Gold OA, avec un licence Creative Commons CC-by pour toutes les publications des auteurs des organismes concernés. ${ }^{68}$
\end{abstract}

\title{
Finlande
}

FinELib, le consortium finlandais pour l'accès à la documentation numérique, a signé avec Elsevier un accord 2018-2020 incluant un développement de la publication en OA des articles des chercheurs finlandais dans les revues du groupe. ${ }^{69} \mathrm{Il}$ ne s'agit cependant que d'une réduction de $50 \%$ réductions sur les APC dans des revues principalement hybrides. ${ }^{70}$

\section{Suisse}

Bien que pratiquant aussi la voie verte, la Suisse a choisi prioritairement le Gold OA. L'Assemblée plénière de swissuniversities, la conférence des recteurs des hautes écoles suisses, a adopté le 31 janvier 2017 une stratégie nationale Open Access. Elle prévoit de rendre librement accessibles toutes les publications financées par les pouvoirs publics en 2024. Elle a engagé en 2018 une négociation globale sur les tarifs des abonnements et des APC avec Springer-Nature, qu'elle étendra à Wiley et Elsevier en 2019. ${ }^{71}$

67. Koen Becking. « Disruptive innovation in scholarly communications ». JSO 2018. 23 jan. 2018. URL : https://jso2018.sciencesconf.org/data/pages/Koen_Becking_2.pdf (visité le 20/02/2018); Open Access.nl. URL : http://www.openaccess.nl/en (visité le 19/02/2018).

68. Ralf Schimmer. « Leveraging Bibliodiversity : Transforming the Journal System and Shifting our Spending from Subscription to Open Access ». JSO 2018. 23 jan. 2018. URL : https : // jso2018. sciencesconf.org/data/pages/Ralf_Schimmer_2.pdf (visité le 09/03/2018).

69. Antoine OuRY. Finlande : Elsevier et les universités signent un accord pour plus d'accès ouvert. 23 jan. 2018. URL : https://www .actualitte.com/article/monde-edition/finlande-elsevier-etles-universites-signent-un-accord-pour-plus-d-acces-ouvert/86895 (visité le 19/02/2018); FinELib's Elsevier agreement and transparency - questions and answers. 2 fév. 2018. URL : http:// finelib.fi/finelibs-elsevier-agreement-and-transparency-questions-and-answers/ (visité le 08/03/2018).

70. Toma SusI. Finland takes steps in the openness of academic journal pricing. 25 jan. 2018. URL : https://www .mostlyphysics.net/blog/2018/1/25/finland-takes-a-step-back-in-the-opennessof-academic-journal-pricing (visité le 08/03/2018).

71. Stratégie nationale suisse Open Access. 31 jan. 2017. URL : https://www.swissuniversities.ch/ $\mathrm{fr} /$ themes/politique-des-hautes-ecoles/open-access/ (visité le 30/04/2018). 


\subsection{Diamant et platine : l'édition électronique en OA}

L'édition électronique en OA est une sorte de retour au sens premier du Gold OA, sans paiement d'APC. ${ }^{72}$ Cela suppose l'existence d'une autre forme de financement, à l'aide de subventions publiques ou de services premium payants, par exemple. Elles portent des noms dont la définition ne fait pas consensus. On parle parfois de voie diamantée (Diamond OA), sans préciser le modèle de financement. L'expression Platinium OA semble désigner un mode de financement dans lequel seul l'accès au texte de l'article est libre, les services complémentaires (fichier PDF, statistiques...) étant payants. Ce serait donc la forme propre à l'OA du freemium ${ }^{73}$.

L'édition électronique ouverte est généralement à l'initiative de structures publiques ou coopératives. Bien qu'il s'agisse de la forme originale de la voie dorée, dans les faits, on ne parle maintenant de voie dorée que dans le cas des éditeurs commerciaux traditionnels, avec APC.

N'étant pas soumise à une obligation de rémunération du capital, l'édition électronique ouverte présente l'avantage d'un coût raisonnable pour les financeurs que sont les institutions publiques, associée cependant à une bonne garantie de qualité, tant au niveau des contenus (peer review) que de leur forme (HTML et PDF de qualité).

\subsubsection{Les grands éditeurs publics}

En France, deux grands éditeurs publics sont prédominants : OpenEdition et Persée.

\section{OpenEdition}

OpenEdition ${ }^{74}$ est un portail de ressources numériques en sciences humaines et sociales. Début 2018, il donnait accès à plus de 5000 livres et à 480 revues. Il s'agit de l'aboutissement de l'initiative de Marin Dacos, qui, en 1999, lança Revues.org, avec deux revues en ligne, depuis sa chambre d'étudiant à l'Université d'Avignon! Le projet se développe et s'institutionnalise en 2007, avec la création du Centre pour l'édition électronique ouverte (CLEO), ${ }^{75}$ unité mixte de services du CNRS, associé aux Universités d'Aix-Marseille et d'Avignon et à l'EHESS. En 2016, OpenEdition est officiellement reconnu comme infrastructure nationale de recherche par le Ministère de l'enseignement supérieur et de la recherche. ${ }^{76}$

Outre les deux plateformes principales consacrées aux livres (OpenEdition Books) et aux revues (OpenEdition Journals, anciennement Revues.org), OpenEdition héberge également des carnets de recherche (Hypothèses) et un calendrier de manifestations scientifiques (Calenda).

Le modèle économique est fondé sur le freemium. L'accès au texte au format HTML est libre, mais des services complémentaires, comme le téléchargement aux formats PDF et ePub, sans DRM ni quota de téléchargement, et des statistiques d'utilisation, sont vendus aux établissements d'ESR. Au-delà de l'intérêt même de ces services "premium",

72. Benjamin CARACo. La voie diamantée du libre accès. Le comptoir de l'accès ouvert - Open access desk. 27 jan. 2014. URL : https://oadesk.hypotheses.org/author/caraco (visité le 01/03/2018).

73. Contraction de free et de premium

74. OpenEdition. URL : http://www.openedition.org/ (visité le 08/03/2018).

75. Centre pour l'édition électronique ouverte. URL : http : / cleo . openedition . org/ (visité le 08/03/2018).

76. Les grandes dates. Centre pour l'édition électronique ouverte. URL : http://cleo.openedition . org/presentation/dates (visité le 08/03/2018). 
l'objectif est surtout de financer l'ensemble de la structure et de pouvoir ainsi pérenniser et développer l'accès libre au texte. Le CLEO résume sa philosophie en deux phrases:

Les biens numériques sont "non-rivaux" et ont des propriétés proches de la connaissance elle-même : en transmettant la connaissance, l'émetteur de celle-ci ne s'en dépossède pas.

$[\ldots]$

Pour OpenEdition, c'est le texte qui est en libre accès, et ce sont les services autour du texte qui sont proposés à la vente aux particuliers et aux bibliothèques. ${ }^{77}$

\section{Persée}

En 2003, le MESR lançait un appel à projet pour constituer un consortium d'établissements pour concevoir et maintenir une plateforme de numérisation, d'enrichissement et de diffusion des collections patrimoniales de revues en sciences humaines et sociales. L'Université Lyon 2 et ses partenaires répondent à cet appel avec le programme «Périodiques scientifiques en édition électronique », qui deviendra célèbre sous le nom de Persée. Le portail Persée ouvre en 2005. ${ }^{78}$ En 2013, Persée devient une unité mixte de service du CNRS, aujourd'hui en partenariat avec la ComUE Université de Lyon et l'ENS de Lyon. En 2014, le champ de Persée s'élargit aux sciences et techniques. En 2016, Persée est officiellement reconnu par le MESR comme infrastructure nationale de recherche. ${ }^{79}$

Mi 2017, Persée contenait plus de 300 collections, totalisant 700000 documents soit numérisés, dont le plus ancien date de 1840, soit nativement au format PDF. ${ }^{80}$

Persée étant entièrement financé sur fonds public, l'accès à ses collections et services est gratuit pour tous, qu'il s'agisse des lecteurs, des bibliothèques ou des éditeurs.

\subsubsection{Les sociétés savantes : EDP Sciences}

Plusieurs sociétés savantes françaises de physique, chimie, mathématiques appliquées et optique se sont groupées pour fonder leur propre maison d'édition, EDP Sciences, nom d'usage pour Edition Diffusion Presse Sciences. ${ }^{81}$

Dans le cadre du plan de soutien à l'édition scientifique française, EDP Sciences a signé un accord 2017-2021 avec le MESRI, Couperin et l'ABES. Cet accord permet aux chercheurs des établissement adhérents de publier en OA dans 29 revues sans payer d'APC. ${ }^{82}$

77. Le libre accès. Centre pour l'édition électronique ouverte. URL : http://cleo.openedition.org/ missions/libre-acces (visité le 08/03/2018), Dans les faits, les services premium ne sont vendus qu'aux institutions.

78. Persée. URL : http://www.persee.fr/ (visité le 08/03/2018).

79. Persée : Historique. URL : http://info.persee.fr/historique/ (visité le 08/03/2018).

80. Persée : À propos. URL : http://www.persee.fr/a-propos (visité le 08/03/2018).

81. EDP Sciences. URL : https://www.edpsciences.org/fr/ (visité le 25/04/2018).

82. EDP Sciences - French academics to benefit from national open access deal with EDP Sciences. 12 déc. 2017. URL : https : / / www . edpsciences . org/fr/actualites / 1645-french-academicsto-benefit-from-national-open-access-deal-with-edp-sciences (visité le 27/04/2018); EDP Sciences - National open access deal in France. URL : https ://www.edpsciences.org/fr/nationalopen-access-deal-in-france (visité le 25/04/2018). 
EDP Sciences publie également en OA une partie de ses publications sur sa plateforme EDP Open. ${ }^{83}$

\subsubsection{Quelques projets étrangers}

Trois projets, originaires du Québec et d'Amérique latine, présentent des points communs avec OpenEdition : Erudit, SciELO et Redalyc.

\section{Erudit}

Fondé en 1998 par l'Université de Montréal, l'Université Laval et l'UQAM, Erudit diffuse par voie électronique plus de 150 revues en sciences humaines et sociales, ainsi que des livres, thèses, mémoires, livres et rapports de recherche. ${ }^{84}$ Erudit annonce $95 \%$ de contenu en libre accès, les $5 \%$ restant, accessible uniquement aux abonnés, correspondant à la dernière année des revues savantes et aux trois dernières années des revues culturelles diffusées. ${ }^{85}$

\section{SciELO}

Créé au Brésil en 1997, SciELO, acronyme de Scientific Electronic Library Online est à la fois une base de données bibliographique et une plateforme coopérative de publications en OA. Son périmètre s'étend aujourd'hui à l'Amérique latine, la Péninsule ibérique et l'Afrique du Sud. ${ }^{86}$

\section{Redalyc}

Redalyc (Red de Revistas Científicas de América Latina y El Caribe, España y Portugal) est également une base de données bibliographique et une plateforme coopérative de publications en OA. Créé en 2002, Redalyc est maintenu par l'Université autonome de l'Etat du Mexique. ${ }^{87}$

\subsubsection{Les revues publiées par les universités}

A une moindre échelle, les universités peuvent aussi tenir le rôle d'éditeur public en OA. Sans prétendre à l'exhaustivité, on pourra citer quelques exemples français.

83. EDP Open. URL : https://www.edp-open.org/.

84. Érudit. URL : http://www.erudit.org/fr/ (visité le 25/04/2018).

85. Érudit : Accès au contenu. URL : https://apropos.erudit.org/fr/bibliotheques/acces-aucontenu/ (visité le 25/04/2018).

86. SciELO.org - Scientific Electronic Library Online. URL : http://www.scielo.org/php/index.php (visité le 08/03/2018), Pays participant à SciELO : Afrique du Sud, Argentine, Bolivie, Brésil, Chili, Colombie, Costa Rica, Cuba, Espagne, Mexique, Paraguay, Pérou, Portugal, Uruguay, Vénézuela.

87. Redalyc. Sistema de Información Científica Redalyc. URL : http://www . redalyc.org/home .oa (visité le 08/03/2018), Pays participant à Redalyc : Argentine, Brésil, Chili, Colombie, Costa Rica, Cuba, Equateur, Espagne, Mexique, Pérou, Porto Rico, Portugal, République dominicaine, Uruguay, Vénézuela. 


\section{Bordeaux}

L'Université de Bordeaux a créé un portail de quatre revues scientifiques. Trois titres sont sous licence Creative Commons, le texte du quatrième, une revue juridique, étant librement accessible, mais sans mention de licence. ${ }^{88}$

\section{Clermont Auvergne}

Le Pôle éditorial numérique (Polen) de l'Université Clermont Auvergne assure l'édition de six revues universitaires, dont deux en partenariat avec OpenEdition. ${ }^{89}$

\section{Lyon 3}

Le SCD de l'Université Jean Moulin Lyon 3 édite les revues des laboratoires de l'établissement. Elle utilise pour cela Lodel, le logiciel d'édition électronique développé par le Cléo pour OpenEdition. La plateforme donnes accès à 13 revues de laboratoires et 6 revues soutenues par Lyon 3 et ses laboratoires. Certains titres sont hébergés sur des sites externes, comme OpenEdition, ou plus rarement sur des sites d'éditeurs commerciaux, en accès payant. ${ }^{90}$

\section{Saint-Etienne}

L'Université Jean Monnet, de Saint-Etienne, publie une revue en OA, Les Cahiers $d u$ CELEC (Centre d'étude sur les langues et les littératures étrangères et comparées). Les textes sont librement accessibles au format HTML, mais, en l'absence de licence formelle, il est mentionné « Nota bene : tous les articles publiés sur le site des Cahiers du CELEC sont protégés au titre du droit d'auteur. Veuillez nous contacter pour toute demande de diffusion ou de téléchargement. ». ${ }^{91}$

\subsubsection{Le Fair Open Access}

Le Fair Open Access (FOA) est une forme d'OA dans lequel la revue est contrôlée par sa communauté scientifique et non par l'éditeur. Le FOA est apparu avec l'émancipation de la revue Lingua de son éditeur traditionnel, Elsevier. Les membres du comité de rédaction ont d'abord essayé de négocier avec Elsevier cinq principes, qui deviendront ceux du FOA :

1. la propriété du titre de la revue doit appartenir à son comité éditorial ou à une société savante

2. les auteurs doivent conserver leurs droits

3. la revue doit être en open access

4. les APC doivent être payés par une agence gouvernementale ou un consortium de bibliothèque, mais pas par les auteurs

88. Portail de revues scientifiques. URL : http : / / open . u-bordeaux . fr / journals/ (visité le 08/03/2018).

89. Revues et Corpus en libre accès de l'Université Clermont Auvergne. URL : http://polen.univbpclermont.fr/revues_polen (visité le 08/03/2018).

90. Revues de Lyon 3. URL : https://revues.univ-lyon3.fr/ (visité le 12/06/2018).

91. Cahiers du CELEC. URL : http://cahierscelec.ish-lyon.cnrs.fr/ (visité le 08/03/2018). 
5. les APC doivent être transparents, d'un niveau raisonnable et proportionnels au travail réel de l'éditeur

La négociation ayant échoué, le comité éditorial de Lingua a démissionné en bloc en 2015 et a fondé sa propre maison d'édition, LingOA ${ }^{92}$ et une nouvelle revue, Glossa. Celle-ci a pris la réputation de Lingua, que la communauté appelle maintenant « zombi Lingua ». Deux autres revues de linguistique ont ensuite rejoint LingOA.

Selon le même principe, le comité éditorial du Journal of Algebraic Combinatorics (JaCo) a démissionné en bloc de Springer Nature pour créer la revue Algebric Combinatorics (AlCo) et la fondation MathOA. ${ }^{93}$ Une opération de même nature a également eu lieu dans le domaine de la psychologie, avec PsyOA. ${ }^{94}$ The Open Library of Humanities $(\mathrm{OLH}),{ }^{95}$ éditeur à but non lucratif, a également adopté le FOA. Selon Johan Rooryck, professeur de linguistique française à l'université de Leiden (Pays-Bas) et président de la Fair open access (FOA) alliance, ${ }^{96}$ le modèle est économiquement soutenable. En adoptant le FOA, le coût d'une revue réputée qui publie 100 articles par an passerait de $800000 €(4000$ bibliothèques abonnées chacune à $2000 €)$ à $100000 €(100$ articles fois $\left.1000 € \mathrm{~d}^{\prime} \mathrm{APC}\right) .{ }^{97}$

\subsection{D'autres formes de revues}

Pour compléter le panorama de l'OA légal, on évoquera brièvement d'autres formes de revues : les mégarevues, les épirevues et les revues prédatrices.

\subsubsection{Les mégarevues}

Une mégarevue ${ }^{98}$ est une forme particulière du Gold OA, généralement sur le modèle auteur-payeur. Sa caractéristique principale est de ne pas se limiter à un champ disciplinaire restreint, mais, au contraire, à publier, exclusivement en ligne, des articles dans de nombreux domaines, tant en SHS qu'en STM. Les articles font l'objet d'une évaluation par les pairs allégée et d'une publication rapide. L'expression « mégarevue » est entrée dans les moeurs. Cependant, en l'absence d'organisation par secteurs disciplinaires pointus, avec un comité éditorial et une politique propres, on peut considérer qu'il s'agit plus d'une base de données d'articles indépendants que d'une vraie revue.

Le concept de mégarevue a été inventé en 2006 par PLOS, avec PLOS ONE. ${ }^{99}$

Fondé en 2012 par un ancien collaborateur de PLOS One, PeerJ ${ }^{100}$ se caractérise par un mode de financement original, dans lequel le chercheur peut éviter les APC en payant une seule fois une cotisation de quelques centaines de dollars, lui donnant le droit de publier à vie. Les articles scientifiques ayant souvent de nombreux auteurs, la

\footnotetext{
92. Ling OA. URL : https://www.lingoa.eu/ (visité le 19/02/2018).

93. MathOA - Converting mathematics journals to open access. URL : http://www.mathoa.org/ (visité le 19/02/2018).

94. PsyOA. URL : http://psyoa.org/ (visité le 19/02/2018).

95. Open Library of Humanities. URL : https://www.openlibhums.org/ (visité le 19/02/2018).

96. Fair Open Access. URL : https://www.fairopenaccess.org/ (visité le 19/02/2018).

97. Johan Rooryck. «Vers un nouveau modèle de publication : Fair Open Access ». JSO 2018. 23 jan. 2018. URL : https://jso2018. sciencesconf.org/data/pages/Paris_Couperin23jan18.pdf (visité le 08/03/2018).

98. mega journal en anglais

99. PLOS ONE : accelerating the publication of peer-reviewed science. URL : http://journals.plos. org/plosone/ (visité le 09/03/2018).

100. PeerJ - Open Access. URL : https://peerj.org/ (visité le 25/04/2018).
} 
publication du premier article dans PeerJ peut être assez onéreuse, le coût relatif baissant au fur et à mesure que le nombre d'articles publiés augmente. ${ }^{101}$

D'autres mégarevues ont été ensuite créées à l'initiative de grands éditeurs comme SAGE, Springer et Elsevier, mais également d'établissements d'enseignement supérieur ou d'organismes de recherche. ${ }^{102}$

\subsubsection{Les épi-revues}

Les épi-revues ${ }^{103}$ sont des revues en $\mathrm{OA}$, avec comité de lecture, créées à partir de preprints déposé dans un dépôt d'archives ouvertes. ${ }^{104} \mathrm{Il}$ s'agit d'une façon originale, mais encore peu répandue, de créer du Gold OA à partir de Green OA :

Episciences.org marie de façon innovante les deux voies du libre accès : la voie dorée par l'hébergement de revues en open access (epi-revues) et la voie verte puisque le processus de soumission des articles à ces revues se fait par un dépôt dans une archive ouverte. ${ }^{105}$

En France, Episciences a été créé par le CCSD en 2013. Episciences est une plateforme permettant à des comités de lecture de sélectionner des preprints déposés dans HAL, afin de les publier dans une épi-revue. ${ }^{106}$ Début 2018, Episciences hébergeait dix épirevues. ${ }^{107}$

Il existe d'autres épi-revues, comme par exemple Symmetry, Integrability and Geometry : Methods and Applications (SIGMA), qui utilise des pré-prints déposés dans arXiv. 108

\subsubsection{Les revues prédatrices}

L'édition scientifique pouvant être une activité très lucrative, des revues prédatrices ${ }^{109}$ sont apparues. Le concept de revue prédatrice a été popularisé par le bibliothécaire américain Jeffrey Beall à partir de 2008, qui a tenté d'en établir la liste jusqu'en 2017. Bien qu'utilisant de nombreux codes des vraies revues scientifiques dans leur communication à destination des chercheurs, elles n'ont pour finalité que d'encaisser des APC, en publiant des articles mal ou non révisés par les pairs, dans des revues sans

101. PeerJ. In : Wikipedia. Page Version ID : 829790753. 10 mar. 2018. URL : https://en.wikipedia. org/wiki/Peer J (visité le 25/04/2018).

102. Thérèse Hammeau. Les mégarevues. 20 fév. 2014. URL : http://openaccess.inist.fr/?Lesmegarevues (visité le 09/03/2018); Mega journal. In : Wikipedia. Page Version ID : 822136229. 24 jan. 2018. URL : https://en.wikipedia.org/w/index.php?title=Mega_journal\&oldid=822136229 (visité le $09 / 03 / 2018$ ).

103. overlay journal en anglais

104. Overlay journal. In : Wikipedia. Page Version ID : 771938195. 24 mar. 2017. URL : https://en. wikipedia.org/w/index.php?title=0verlay_journal\&oldid=771938195 (visité le 09/03/2018).

105. Episciences. URL : https://www.episciences.org/ (visité le 19/02/2018).

106. Christine Berthaud et al. «EPISCIENCES - an overlay publication platform ». In : Let's Put Data to Use : Digital Scholarship for the Next Generation. 18th International Conference on Electronic Publishing. Thessaloniki, Greece, 2014, p. 78-87. DOI : 10.3233/978-1-61499-409-1-78. (Visité le 09/03/2018); Agnès Magron. Catégorie "Episciences". URL : https://www.ccsd.cnrs.fr/category/ episciences/ (visité le 09/03/2018).

107. Epi-revues. URL : https://www.ccsd.cnrs.fr/epi-revues/ (visité le 19/02/2018).

108. SIGMA (Symmetry, Integrability and Geometry : Methods and Applications). URL : http://www. emis.de/journals/SIGMA/ (visité le 09/03/2018).

109. predatory journal ou rogue journal en anglais 
notoriété. Au mieux, le chercheur s'est fait délester du paiement des APC. Au pire, il entache sa réputation et celle de son institution. ${ }^{110}$

Les revues prédatrices ont habituellement une politique commerciale agressive. Elles envoient des courriels flatteurs aux chercheurs, leur promettant une publication rapide pour un coût raisonnable. Certaines revues prédatrices ont un titre qui ressemble beaucoup à celui d'une revue réputée dans sa discipline. Parfois, les revues prédatrices invitent les chercheurs à des colloques prédateurs, dans le seul but de leur soutirer des droits d'inscription.

Il y aurait plusieurs milliers de revues prédatrices dans le monde. Il existe des guides pour les repérer. ${ }^{111}$

\subsection{Grey : la zone grise}

Bien qu'aucune couleur ne soit officiellement attribuée à ce type de pratiques, il nous a semblé que le gris convenait bien à leur flou en matière de droit et de pérennité.

\subsubsection{Les réseaux sociaux académiques}

Les réseaux sociaux académiques $(\mathrm{RSA})^{112}$, se résument essentiellement à Academia.edu et ResearchGate. ${ }^{113}$ Bien que ne faisant pas partie à proprement parler du mouvement de l'Open Access, ils permettent aux chercheur d'y déposer les PDF de leurs publications.

Tous deux fondés en 2008, Academia.edu est originaire des Etats-Unis et plutôt orienté SHS, tandis que ResearchGate est né en Allemagne et se veut pluridisciplinaire, avec une prédominance des STM. Les revues ont longtemps toléré le dépôt de PDF éditeurs sur les RSA. Devant l'inflation du nombre d'articles librement accessibles, plusieurs éditeurs ont demandé aux RSA de dépublier les articles sous copyright. En 2017, une association des principaux éditeurs en STM a intenté un procès contre ResearchGate, qui a dépublié 2 millions d'articles. ${ }^{114}$

Il importe donc de bien sensibiliser les chercheurs au fait que le dépôt dans un RSA n'est pas un dépôt en archive ouverte. Ce type de dépôt fait perdre au chercheur le contrôle de l'usage de ses articles, lui fait prendre un risque d'illégalité, et n'offre aucune garantie de pérennité de la présence en ligne de ses PDF. Ces questions sont développées dans la troisième partie du présent rapport.

110. Predatory open access publishing. In : Wikipedia. Page Version ID : 828044570. 28 fév. 2018. URL : https : / / en . wikipedia _org/wiki / Predatory_open_access_publishing (visité le 09/03/2018); Sylvestre Huet. Open access : du rêve au cauchemar. 4 oct. 2013. URL : http://sciences . blogs . liberation.fr/2013/10/04/open-access-du-reve-au-cauchemar-/ (visité le 09/03/2018); Sylvestre HuET. Open access : du rêve au cauchemar? L'avis de J-C Guédon. 4 oct. 2013. uRL : http://sciences . blogs.liberation.fr/2013/10/04/open-access-du-reve-au-cauchemar-bis/ (visité le 09/03/2018). 111. Cécile Fovet-RAвот. Eviter les éditeurs prédateurs. 2017. URL : http://coop-ist.cirad.fr/ editeurs-predateurs (visité le 09/03/2018); Université de RENNES 1. Les revues prédatrices. 11 mai 2016. URL : https://openaccess.univ-rennes1.fr/les-revues-predatrices (visité le 09/03/2018); Stop Predatory Journals. URL : https://predatoryjournals.com/ (visité le 09/03/2018).

112. On parle aussi de réseaux sociaux de chercheurs ou de réseaux sociaux de recherche 113. Academia.edu. URL : https : //www . academia.edu/ (visité le 12/03/2018); ResearchGate. URL : https://www.researchgate.net/ (visité le 12/03/2018).

114. Aline Bouchard. "Nous n'avons pas d'autre choix": les éditeurs, ResearchGate et les guerres du copyright. 6 nov. 2017. URL : https://urfistinfo.hypotheses.org/3126 (visité le 16/04/2018). 


\subsubsection{Unpaywall}

Unpaywall est un module complémentaire pour Firefox permettant de savoir s'il existe une version gratuite d'une publication payante qu'on cherche à lire et, le cas échéant d'y accéder. Selon Unpaywall, cette extension permettrait de lire gratuitement et en toute légalité plus de la moitié des articles scientifiques publiés en ligne. ${ }^{115}$

\subsubsection{Lazy Scholar}

Lazy Scholar est un module complémentaire pour Firefox et Chrome. Il s'agit d'un métamoteur, qui cherche du texte intégral en accès libre et des métriques dans plusieurs moteurs, dont Google Scholar et PubMed. Lazy Scholar est développé par un doctorant, Colby Vorland. ${ }^{116}$

\subsubsection{Open Acces Button}

Créé en 2013, Open Acces Button est un outil de recherche d'articles en libre accès. Open Acces Button cherche d'abord si l'article demandé est disponible en libre accès. Dans le cas contraire, il envoie une requête à l'auteur, accompagnée de conseils pour mettre son article en libre accès. Les requêtes sont publiques et toutes les personnes intéressées par un article sont invitées à s'y associer, pour motiver l'auteur à répondre. ${ }^{117}$

\subsubsection{Kopernio}

Kopernio est un module complémentaire pour Chrome, Firefox et Opera, qui déclare chercher « la meilleure version PDF disponible d'un article de recherche », c'est-à-dire la version finale publiée si elle est librement accessible. A défaut, Kopernio cherche une version alternative.

A la différence d'Unpaywall, Lazy Scholar et Open Access Button, qui sont des projets sans but lucratif, Kopernio, bien que gratuit, appartient à Clarivate Analytics, qui possède le Web of Science, EndNote et le Journal Impact Factor. ${ }^{118}$

Unpaywall, Lazy Scholar, Open Acces Button et Kopernio sont des outils utiles pour accéder librement à l'information scientifique, ${ }^{119}$ mais à condition que celle-ci ait été au préalable publiée en OA par un tiers. Ils ne dispensent donc pas les chercheurs et les institutions de leur effort pour le développement de l'OA.

\subsection{Black : les solutions illégales}

Les solutions illégales sont très employées, y compris dans les pays riches. Si les institutions publiques ne peuvent évidemment pas les encourager, leur existence est

115. Unpaywall. URL : http://unpaywall.org/ (visité le 19/02/2018).

116. Lazy Scholar. URL : http://www.lazyscholar.org/ (visité le 25/04/2018).

117. Open Access Button. URL : https://openaccessbutton.org/.

118. Kopernio. URL : https://kopernio.com (visité le 18/10/2018).

119. Des extensions de navigateurs pour repérer les articles en libre-accès - BU Lyon 1 - Université Lyon 1. BU Lyon 1. URL : https://portaildoc.univ-lyon1.fr/chercheur/des-extensions-denavigateurs-pour-reperer-les-articles-en-libre-acces-944526.kjsp (visité le 06/12/2018). 
néanmoins la preuve des tensions que provoque l'importance du nombre de publications à accès restreint, à un tarif prohibitif pour de très nombreux lecteurs.

\subsection{1 \#ICanHazPDF}

Initié par le chercheur Andrea Kuszewski, \#ICanHazPDF représente la forme artisanale du Black OA. Elle consiste à demander sur Twitter la copie intégrale d'un article payant, en envoyant un tweet contenant le hastag \#ICanHazPDF. Une fois l'article reçu, le tweet est généralement supprimé par son auteur. ${ }^{120}$

\subsubsection{Sci-Hub}

La plus célèbre des solutions illégales est Sci-Hub. ${ }^{121}$ A la différence \#ICanHazPDF, Sci-Hub est une forme d'industrialisation du Black OA. ${ }^{122}$ Sci-Hub a été créé en 2011 par une étudiante kazakhe, Alexandra Elbakyan, afin de donner au plus grand nombre un accès gratuit aux publications scientifiques, en particulier dans les pays pauvres. Les publications disponibles sur Sci-Hub ont été piratées sur les serveurs de leurs éditeurs, à partir d'établissements d'enseignement supérieur et de recherche disposant d'abonnements légaux. Elle représentent une part importante du fonds des grands éditeurs commerciaux. ${ }^{123}$ Sci-Hub a été attaqué en justice par Elsevier en 2015, puis par l'American Chemical Society en 2017. Cependant, Sci-Hub étant hébergé en Russie, où Alexandra Elbakyan s'est réfugiée pour éviter d'être extradée aux Etats-Unis, le site continue a fonctionner malgré les condamnations des tribunaux américains. ${ }^{124}$

Une étude publiée en 2016 à partir des données de consultation d'une période de six mois a montré que Sci-Hub était utilisé par les chercheurs du monde entier, y compris ceux des pays riches travaillant dans des institutions abonnées aux principaux bouquets numériques payants, principalement pour le confort d'utilisation d'une interface unique leur permettant d'interroger un très grand nombre de revues avec une seule requête. ${ }^{125}$

\subsubsection{LibGen}

Moins connu, Library Genesis, ou LibGen, fonctionne sur le même principe que SciHub et a également fait l'objet de condamnations en justice aux Etats-Unis. ${ }^{126}$

120. ICanHazPDF. In : Wikipedia. Page Version ID : 805921107. 18 oct. 2017. URL : https : //en . wikipedia .org/w/index . php?title=ICanHazPDF\&oldid=805921107 (visité le 19/02/2018); Twitter : ICanHazPDF. URL : https://twitter.com/hashtag/icanhazpdf; "The scientists encouraging online piracy with a secret codeword ». In : BBC News (21 oct. 2015). URL : http://www.bbc.com/news/blogstrending-34572462 (visité le 14/03/2018).

121. Sci-Hub. In : Wikipédia. Page Version ID : 146185697. 7 mar. 2018. URL : https://fr.wikipedia. org/w/index $\cdot$ php?title=Sci-Hub\&oldid=146185697 (visité le 12/03/2018).

122. «Sci-Hub, le Pirate Bay de la recherche scientifique, est de retour ». In : Le Monde.fr (15 fév. 2016). ISSN : 1950-6244. URL : http : / www. lemonde.fr/pixels/article/2016/02/15/sci-huble-pirate-bay-de-la-recherche-scientifique-est-de-retour_4865611_4408996.html (visité le 14/03/2018).

123. Sci-Hub Stats Browser. URL : https://greenelab.github.io/scihub/\#/ (visité le 19/02/2018).

124. Sci-Hub / Academic Search Engine - To remove all barriers in the way of science. URL : http: //sci-hub.tech/ (visité le 12/03/2018), Sci-Hub utilise plusieurs noms de domaine et en change parfois lorsque certains lui sont interdits par décision de justice.

125. John Bohannon. "Who's downloading pirated papers? Everyone ». In : Science (25 avr. 2016). DOI : $10.1126 /$ science.aaf5664. URL : http://www.sciencemag.org/news/2016/04/whos-downloadin g-pirated-papers-everyone (visité le 12/03/2018).

126. Library Genesis 2M. URL : http://gen.lib.rus.ec/ (visité le 19/02/2018); Library Genesis. In : Wikipédia. Page Version ID : 141911763. 25 oct. 2017. URL : https : //fr . wikipedia .org/w/ 
Selon le point de vue, Sci-Hub et LibGen peuvent être perçus comme une atteinte au droit légitime de propriété intellectuelle ou comme une entreprise mondiale d'émancipation de la science. ${ }^{127}$ Bien évidemment, aucun pays ou institution ne peut en faire la base d'une politique d'OA.

\subsection{De l'Open Access à la Science ouverte}

Bien que n'entrant pas strictement dans l'objet du présent rapport, on ne peut passer sous silence la science ouverte ${ }^{128}$, qui est le prolongement naturel de l'Open Access.

La science ouverte est la généralisation à toutes les étapes de la production scientifique des principes de l'OA. ${ }^{129}$ Les bibliothèques sont particulièrement concernées par le travail collaboratif sur les preprints, la publication des données de la recherche, la citation ouverte et l'évaluation ouverte.

\subsubsection{Travail collaboratif sur les preprints}

Au-delà de leur seul rôle au sein du Green OA, les dépôts ouverts de preprints peuvent changer considérablement le fonctionnement du peer reviewing. Dans le processus traditionnel, l'article soumis n'est relu que par un nombre limité de réviseurs, souvent selon le principe du « double aveugle », dans lequel les réviseurs ne connaissent pas le nom de l'auteur de l'article et l'auteur ne connaît pas le nom des réviseurs. Dans la procédure ouverte, les preprints sont exposés et soumis à la critique de l'ensemble de la communauté scientifique, la décision finale revenant cependant au comité éditorial de la revue. Ce mode de fonctionnement a été adopté par ASAPbio ${ }^{130}$ et SciPost. ${ }^{131}$

S'il offre un progrès en matière de transparence, de richesse et de rapidité du processus, le peer reviwing ouvert fait aussi l'objet de critiques de la part des tenants du processus traditionnel.

\subsubsection{Publication des données de la recherche}

La publication des données de la recherche est une opération indispensable pour assurer la reproductibilité de la démarche scientifique et, le cas échéant, la confirmation

index $\cdot$ php?title=Library_Genesis\&oldid=141911763 (visité le 19/02/2018); Guillaume CABANAC. "Bibliogifts in LibGen? A study of a text-sharing platform driven by biblioleaks and crowdsourcing ». In : Journal of the Association for Information Science and Technology 67.4 (2016), p. 874-884. ISSN : 2330-1643. DOI : 10.1002/asi.23445. URL : http://dx.doi.org/10.1002/asi.23445; LibGen utilise plusieurs noms de domaine et en change parfois lorsque certains lui sont interdits par décision de justice. Un site permet de retrouver les URL actives : Library Genesis / LibGen - The Meta Library. URL : https://sites.google.com/site/themetalibrary/library-genesis (visité le 19/02/2018).

127. En solidarité avec Library Genesis et Sci-Hub. URL : http: / / custodians . online (visité le 19/02/2018); Florence Piron. Guide de la recherche documentaire dans le web scientifique libre. 2018. URL : https : //www.projetsoha.org/?page_id=1040 (visité le 16/04/2018), Étape 6. Pas assez de résultats?

128. Open science en anglais

129. Le portail FOSTER regroupe des ressources pour l'apprentisage des concepts et le développement de la science ouverte FOSTER. URL : https://www.fosteropenscience.eu/ (visité le 22/03/2018).

130. ASAPbio / Accelerating Science and Publication in Biology. URL : http://asapbio.org/ (visité le 22/03/2018).

131. SciPost. URL : https://scipost.org/ (visité le 22/03/2018). 
ou la réfutation des conclusions exposées dans un article. Publier et conserver ces données est une application spécifique des compétences génériques des professionnels de l'IST. Le projet DoRANum (Données de la Recherche : Apprentissage NUMérique à la gestion et au partage), initié par le réseau des Urfist et l'Inist-CNRS est un outil de formation en ligne à la gestion et du partage des données de la recherche. ${ }^{132}$

\subsubsection{Citation ouverte}

La citation ouverte consite à structurer les données de citation pour faciliter leur partage. Elle est promue par l'Initiative for Open Citations (I4OC). ${ }^{133}$ Début 2018, la moitié des références de Crossref étaient au format OpenCitation, l'objectif étant d'arriver à la totalité. ${ }^{134}$

\subsubsection{Evaluation ouverte}

L'évaluation ouverte cherche à sortir de la bibliométrie traditionnelle et du très critiqué facteur d'impact. Les Altmetrics tiennent ainsi compte d'autres paramètres que le seul nombre de citations d'un article : vues et téléchargements, mentions sur les réseaux sociaux et dans la presse non scientifique. ${ }^{135}$

La Commission européenne a commandé le rapport «Evaluation of Research Careers fully acknowledging Open Science practices » à un groupe de travail, dont Bernard Rentier, pionnier de l'OA lorsqu'il était recteur de l'Université de Liège, a été un des piliers. Ce rapport propose de passer d'un indicateur unique, simple mais trompeur, le facteur d'impact, à une approche multicritères (23 critères en 6 catégories), avec des poids différents. ${ }^{136}$

On observera avec intérêt l'évolution des journées d'étude organisées par Couperin. Lors de la première édition en 2005, on parlait de Journée Archives Ouvertes. A partir de 2013, on est passé aux Journées Open Access. L'édition 2018 s'appelait Journées Science Ouverte. Lors de cette manifestation, Alain Beretz, directeur général de la recherche et de l'innovation (DGRI) au Ministère de l'Enseignement Supérieur, de la recherche et de l'Innovation, a déclaré que le ministère avait la volonté de saisir l'opportunité de la science ouverte à bras le corps, et que les chercheurs, bibliothécaires, informaticiens et juristes devaient travailler ensemble.

On notera également que, depuis 2017, un poste de conseiller scientifique pour la Science Ouverte a été créé auprès du DGRI et confié à Marin Dacos, le fondateur d'OpenEdition. ${ }^{137}$

132. DoRANum - Données de la recherche : Apprentissage Numérique. URL : http://doranum.fr/ (visité le 19/02/2018).

133. Initiative for Open Citations. URL : https://i4oc.org/.

134. Mark PAtTERson. « Setting your cites on open : The Initiative for Open Citations ». JSO 2018. 24 jan. 2018. URL : https://jso2018. sciencesconf .org/data/pages/Mark_Patterson.pdf (visité le $22 / 03 / 2018)$.

135. Altmetrics : a manifesto. URL : http://altmetrics.org/manifesto/ (visité le 22/03/2018).

136. Conor O'CARroll et Bernard Rentier. Evaluation of Research, Careers fully acknowledging, Open Science practices. Commission européenne, 2017. URL : https://cdn1. euraxess.org/sites/defa ult/files/policy_library/os-rewards-wgreport-final_integrated_0.pdf (visité le 19/02/2018).

137. Larousserie, « Marin Dacos, héraut de la science ouverte », op. cit. 
En mars 2018, la Bibliothèque scientifique numérique (BSN) s'est transformée en Comité pour la Science ouverte (CoSO). ${ }^{138}$

Enfin, le 4 juillet 2018, la ministre Frédérique Vidal a prononcé le premier discours officiel sur science ouverte, annonçant une politique nationale articulée autour de la généralisation de l'ouverture des publications et des données de la recherche, dans une dynamique durable, européenne et internationale. ${ }^{139}$

Les bibliothèques ont abordé la science ouverte sous l'angle des publications en OA, mais elles commencent à être concernées par tous ses autres aspects, qui prendront nécessairement de l'ampleur dans les années à venir.

138. AMI pour la constitution du Comité pour la Science Ouverte (CoSO). URL : http://www.bibl iothequescientifiquenumerique. fr/ami-pour-la-constitution-du-comite-pour-la-scienceouverte-coso/ (visité le 26/03/2018).

139. Plan national pour la science ouverte, op. cit. 


\section{Chapitre 2}

\section{Etat des lieux}

Afin d'avoir une vision du développement de l'OA dans le monde de l'ESR français, ce chapitre tente de faire un état des lieux. Seront étudiés successivement les portails HAL et les archives ouvertes institutionnelles, les initiatives académiques en faveur de l'OA, les manifestations organisées à l'occasion de l'Open Access Week et les journées d'étude consacrées à l'OA.

\subsection{HAL et les archives ouvertes institutionnelles}

Quel est le niveau de développement des archives ouvertes dans le monde de la recherche en France? Pour répondre à cette question, une liste de 195 établissements d'enseignement supérieur et de recherche, d'associations de sites universitaires et d'organismes de recherche a été constituée du 21 au 30 novembre 2017. On a ensuite recherché si ces établissements disposaient d'une archive ouverte, qu'il s'agisse d'un portail ou d'une collection dans HAL ou d'une archive ouverte institutionnelles (AOI). Le corpus a été actualisé du 29 novembre au 4 décembre 2018. La méthode de constitution du corpus et de recherche des archives ouvertes, ainsi que les données pour chaque établissement constituant le corpus sont détaillées en annexe.

Cette collecte a permis de constituer un embryon de base de données, à partir du quel ont été établis les graphiques ci-après. La lecture de l'enquête, plus qualitative, menée par Couperin en 2017 sur les archives ouvertes en France, éclaire utilement ce recensement. ${ }^{1}$

1. Enquête Archives Ouvertes COUPERIN 2017 : Résultats de l'enquête. Oct. 2017. URL : https: //www . couperin.org/images/stories/A0/Depouillement_enquete_A02017_FINAL_V7 .pdf (visité le $30 / 04 / 2018)$. 
Ouvertures de portails HAL ou d'AOI

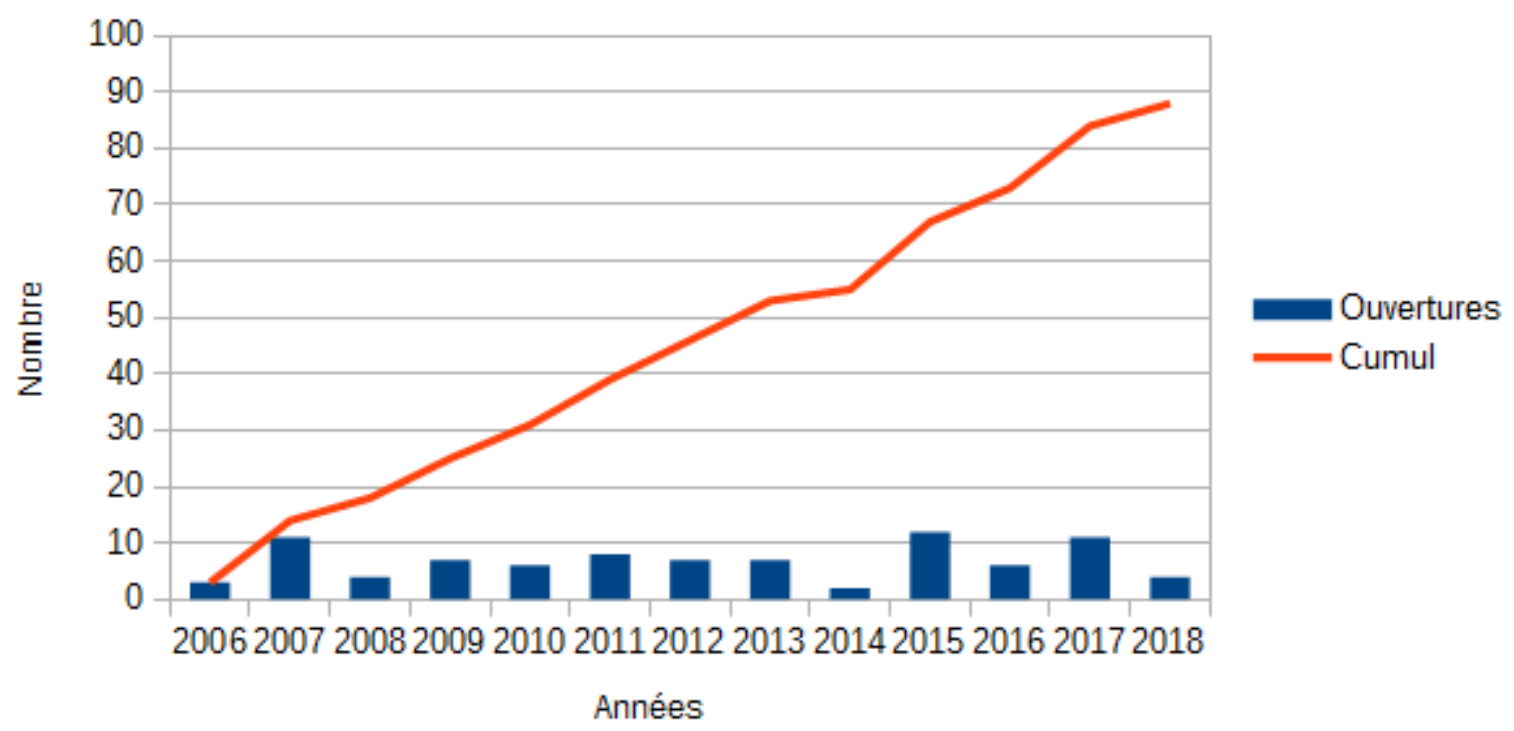

Depuis plus de 10 ans, l'ouverture de portails dans HAL et la création d'AOI se poursuivent de manière constante et linéaire.

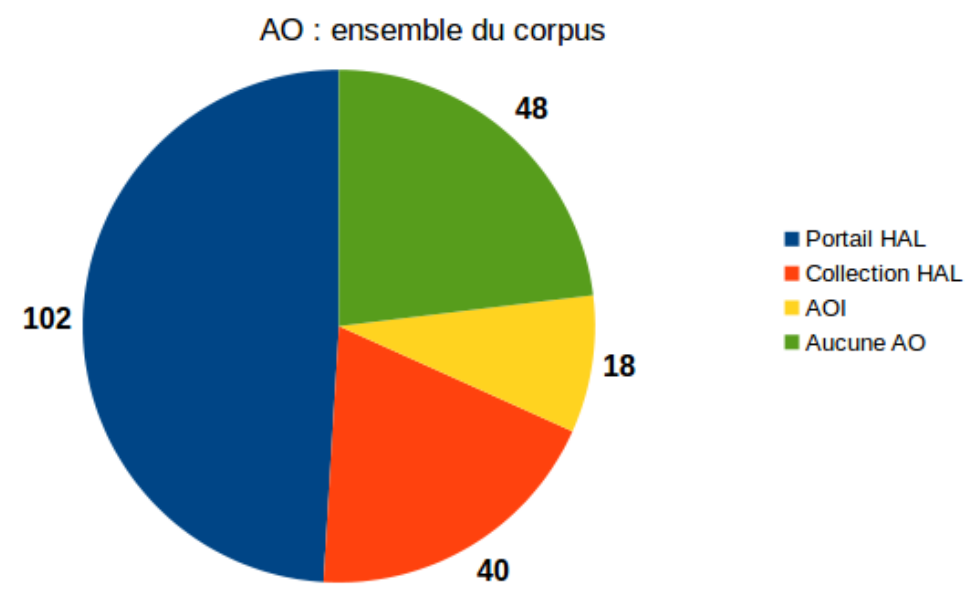

L'usage de HAL, sous la forme de portail ou de collection, est prédominant sur l'ensemble du corpus. Un peu moins du quart des établissements étudiés n'avait pas d'archive ouverte à la date du recensement. Certains en avaient cependant le projet à plus ou moins brève échéance.

Il est nécessaire de détailler cette répartition par type d'établissement. 


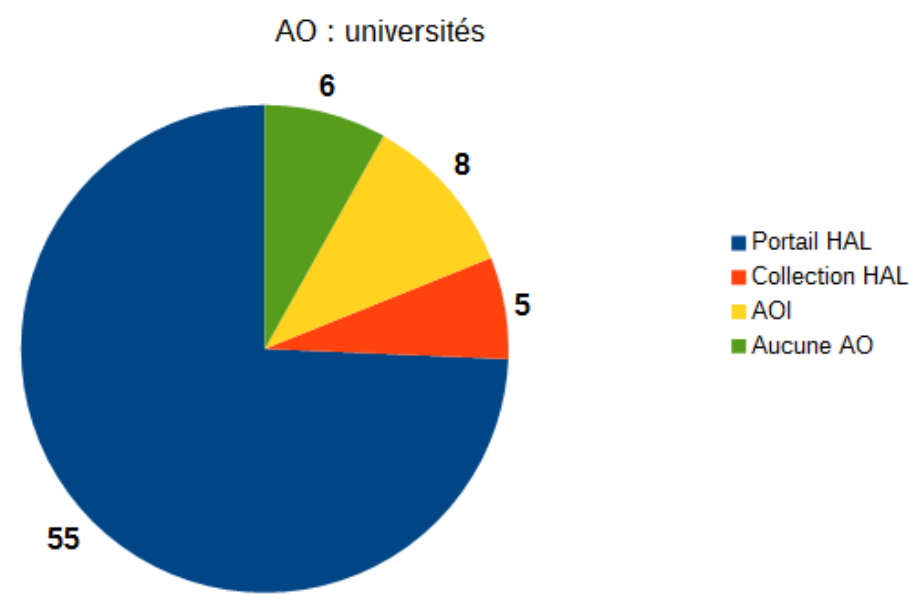

Près des trois quarts des universités ont un portail HAL. Seules huit ont une AOI et six n'ont pas d'archive ouverte.

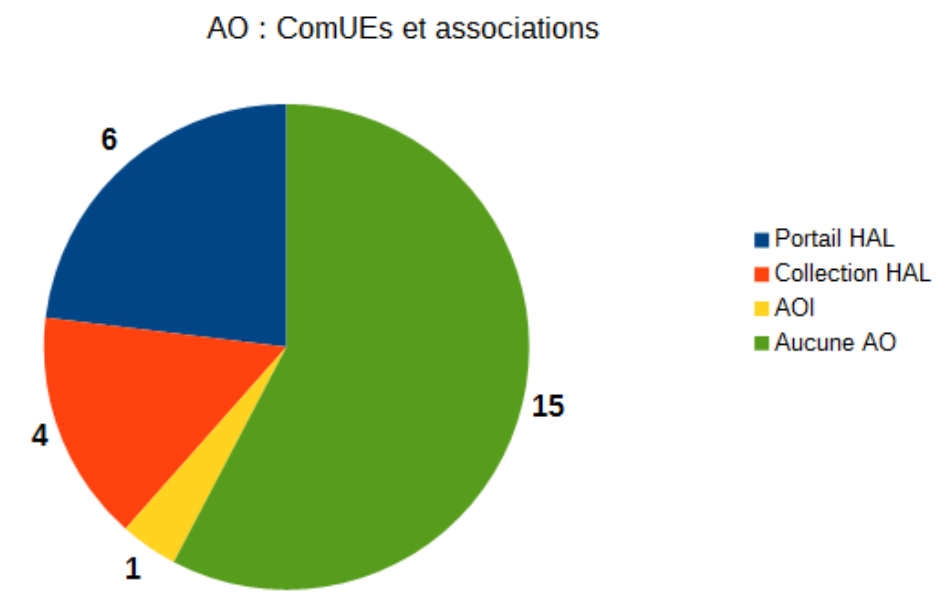

Les ComUEs se sont peu emparées des archives ouvertes. Seules six ont un portail dans HAL. Normandie Universités est la seule à avoir créé un portail pour le compte de tous ses membres. Dans les autres cas, un ou plusieurs membres avaient un portail préexistant à celui de la ComUE. L'association du site alsacien est la seule à porter une AOI. 
AO : autres établissments d'ESR

(Instituts et écoles, ENS, grands établissements, ENSI et ENI, IEP)

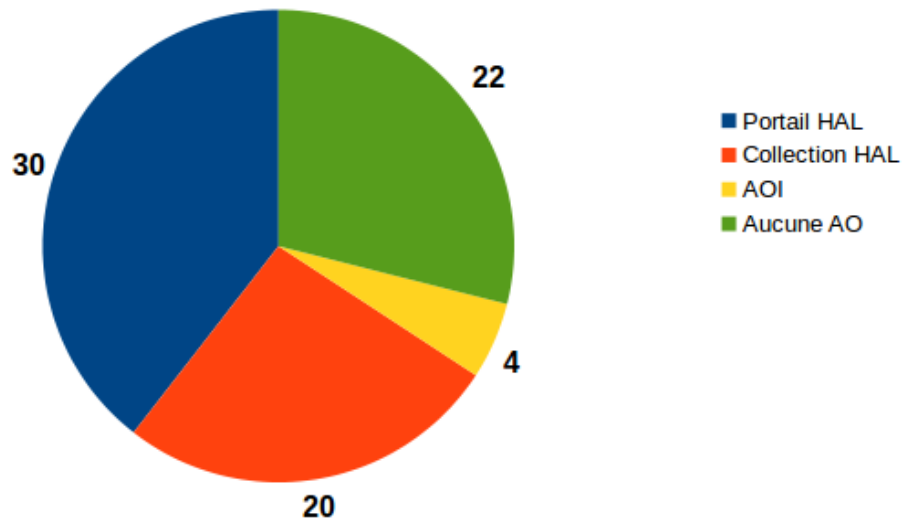

La situation des autres établissements d'enseignement supérieur est contrastée. La part des portails et collections dans HAL est prédominante et près d'un tiers n'a pas d'archive ouverte.

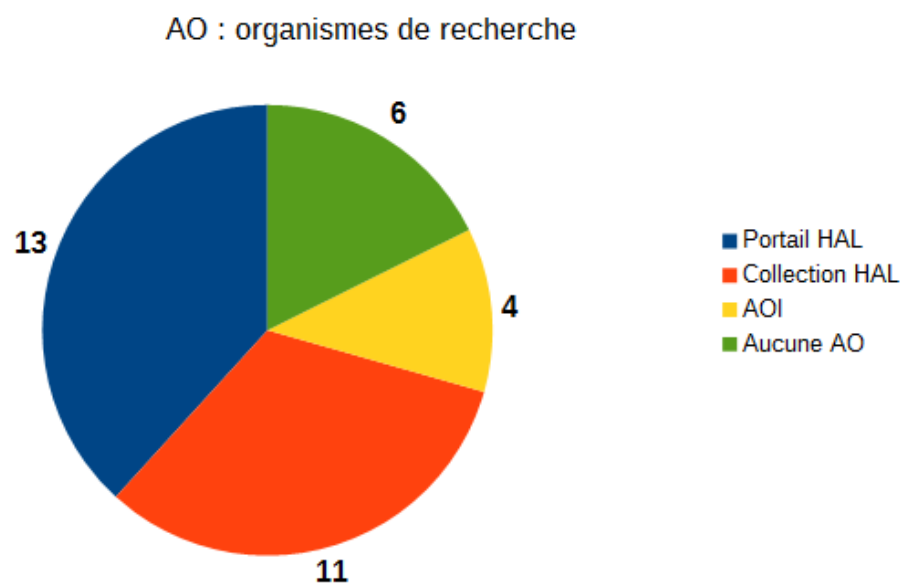

Les organismes de recherche utilisent très majoritairement HAL, les portails et les collections faisant jeu égal. Certains ont cependant développé une importante OAI. Un peu moins du cinquième n'ont pas d'archive ouverte.

\subsection{Initiatives académiques en faveur de l'OA}

A côté des dépôts d'archives ouvertes, certains établissements d'ESR ont pris d'autres initiatives en faveur de l'OA. 


\subsubsection{Mandat de libre accès}

Le mandat de libre accès ${ }^{2}$ est l'obligation faite par une institution à ses membres de publier leur production scientifique en OA, soit dans un dépôt d'archive ouverte, soit dans une revue en $\mathrm{OA}$, soit les deux à la fois.

Plusieurs sources donnent des informations sur la situation française, mais aucune n'est complète et à jour. De nombreux liens vers les politiques de dépôt sont absents ou morts.

Le Registry of Open Access Repository Mandates and Policies (ROARMAP) recense 23 organisations de recherche ou de financement de la recherche qui ont une politique de mandat de libre accès. Dans de nombreux cas, il n'y a aucun lien vers la source juridique du mandat et l'OA semble être une incitation et non une obligation. De plus, certaines institutions ayant plusieurs branches sont comptées plusieurs fois. Et bien que n'ayant aucun pourvoir normatif, l'ADBU figure dans la liste des institutions de recherche! ${ }^{3}$

Sherpa Juliet ne mentionne que l'ANR et le Consortium EurOcean, dont fait partie l'IFREMER. ${ }^{4}$

Enfin, une Synthèse sur les politiques institutionnelles de libre accès à la recherche, réalisée en 2013 par Laurent Jonchère pour l'Université de Rennes 1, donne des informations complémentaires. ${ }^{5}$

Le croisement de ces trois sources, associé à des vérifications en ligne, permet d'établir, sans garantie d'exhaustivité, une liste d'à peine plus de dix établissements et organismes ayant adopté un mandat de libre accès, dont seulement trois universités.

1. Agence Nationale de la recherche $(\mathrm{ANR})^{6}$

2. Arts et Métiers ParisTech ${ }^{7}$

3. Ecole des Ponts ParisTech ${ }^{8}$

4. École Normale Supérieure ${ }^{9}$

5. Institut national de recherche en informatique et en automatique (INRIA) ${ }^{10}$

6. Institut Pasteur ${ }^{11}$

2. Open-access mandate en anglais. L'expression « Mandat de dépôt », trop souvent employée en français, a l'inconvénient de désigner en droit pénal un ordre d'incarcération! L'expression « Mandat de libre accès » doit donc être préférée.

3. RoArmap. Registry of Open Access Repository Mandates and Policies (ROARMAP). 2018. URL : http://roarmap.eprints.org/ (visité le 17/02/2018).

4. Sherpa Juliet. URL : http://v2. sherpa.ac.uk/juliet/ (visité le 23/03/2018).

5. Laurent JONCHÈRE. « Projet d'archive ouverte Université de Rennes 1 : Synthèse sur les politiques institutionnelles de libre accès à la recherche ». In : (2013), p. 36. URL : https://archivesic.ccsd . cnrs.fr/sic_00794497v2.

6. L'ANR incite les chercheurs à intégrer leurs publications dans le système d'archives ouvertes. URL : http://www. agence-nationale-recherche.fr/informations/actualites/detail/?tx_ttnews\%5btt_ news $\% 5 d=159$ (visité le 23/03/2018).

7. Guide du déposant : Guide d'utilisation de l'archive ouverte SAM à l'attention des chercheurs ENSAM. SAM : Science Arts \& Métiers. Version 2.1. 8 juil. 2013. URL : http://sam.ensam.eu/help.html (visité le 23/03/2018).

8. Ecole des Ponts ParisTech : Mandat pour le partage de la science. 27 fév. 2017. URL : https://es pacechercheurs.enpc.fr/sites/default/files/MandatOA_EcoledesPonts.pdf (visité le 23/03/2018).

9. Résolution votée par le Conseil scientifique de l'École normale supérieure sur l'accès libre aux publications. 17 juin 2016. URL : https ://www.eleves.ens.fr/home/cacs/documents-cs/2016-0617/resolution-oa-ens.pdf (visité le 23/03/2018).

10. InRIA. Inria défend l'Open Access. 6 nov. 2015. URL : https://www . inria fr/actualite/ actualites-inria/inria-defend-1-open-access (visité le 23/03/2018).

11. Institut Pasteur - Médiathèque. URL : https ://webext.pasteur.fr/biblio/ressources/oai/ (visité le 23/03/2018). 
7. Institut français de recherche pour l'exploitation de la mer (IFREMER) ${ }^{12}$

8. Institut français des sciences et technologies des transports, de l'aménagement et des réseaux (IFSTTAR) ${ }^{13}$

9. Université d'Angers ${ }^{14}$

10. Université de Rennes $1^{15}$

11. Université Savoie Mont Blanc. ${ }^{16}$

D'une façon générale, on remarquera le style peu juridique et très contextualisé de ces mandats : même quand le dépôt est obligatoire, les établissements veillent à ne pas donner aux chercheurs l'impression qu'une obligation s'impose à eux. Cela est manifestement dû à la contestation de cette obligation par nombre de chercheurs, qui y voient une restriction de leur liberté académique, voire un «flicage » à des fins d'évaluation de leur activité.

A titre d'information, on peut ajouter à cette liste l'UNESCO, qui n'est pas un organisme de recherche français, mais qui a son siège en France. ${ }^{17}$

\subsubsection{Services autour de l'OA}

A défaut d'une enquête systématique, on pourra citer quelques cas intéressants de services associant professionnels de l'IST et chercheurs.

\section{Le GTAO Couperin}

Le Groupe de travail accès ouvert de Couperin est la seule initiative mutualisée. ${ }^{18}$ Le GTAO regroupe plusieurs dizaines de personnes, issues essentiellement des bibliothèques et services de documentation de l'ESR. Il est organisé en quatre sous groupes :

- Spécifications fonctionnelles des archives ouvertes

- Communication, information, formation

- Etudes, enquêtes, recensement des bonnes pratiques

- Juridique (contrats, addendum, relations éditeur)

Le GTAO est à l'origine des journées d'étude Open Access, puis Science ouverte de Couperin et du site web Open Access France. ${ }^{19}$

En mars 2018, il envisageait de se renommer Groupe de travail science ouverte (GTSO Couperin).

12. Obligation de dépôt de la documentation Ifremer dans Archimer. URL : http://archimer.ifremer. fr/html/depot.htm (visité le 23/03/2018).

13. Mandat pour l a diffusion en libre accès. 31 juil. 2017. URL : http://www.ifsttar.fr/fileadmin/ redaction/5_ressources-en-ligne/politiques/DS_DIST_2017_IFSTTAR_mandat_OA_et_depot_ Madis.pdf (visité le 23/03/2018).

14. Politique de dépôt / Okina. URL : http://okina.univ-angers.fr/politique-de-depot (visité le 23/03/2018).

15. La politique open access de Rennes 1. 9 juin 2016. URL : https://openaccess.univ-rennes1.fr/ la-politique-open-access-de-rennes-1 (visité le 28/03/2018).

16. Délibération $n^{\circ} 6$ du conseil d'administration de l'Université Savoie Mont Blanc du 21 novembre 2017 : Dépôt des publications dans HAL. 30 nov. 2017.

17. Open Access Policy concerning UNESCO publications. 31 juil. 2013. URL : http://www . unesco. org/new/fileadmin/MULTIMEDIA/HQ/ERI/pdf/oa_policy_rev2.pdf (visité le 23/03/2018).

18. Open Access - GTAO. URL : http ://www . couperin org/services-et-prospective/openaccess/gtao (visité le 19/02/2018).

19. Open Access France - Le site couperin de l'accès ouvert en France. URL : http://openaccess . couperin.org/ (visité le 19/02/2018). 


\section{L'écosystème toulousain}

Un « écosystème OA » s'est développé autour du site de Toulouse.

Tous les établissements d'enseignement supérieur y ont leur archive ouverte, soit sous forme de portail HAL, soit sous forme d'AOI.

Le taux de dépôt en texte intégral est particulièrement élevé (entre 85 et 100\%) sur OATAO, l'AOI commune à l'Institut National Polytechnique de Toulouse (INPT), l'École Nationale Vétérinaire de Toulouse (ENVT), l'Institut Supérieur de l'Aéronautique et de l'Espace (ISAE-SUPAERO) et l'École Nationale Supérieure de Formation de l'Enseignement Agricole (ENSFEA).

Depuis mars 2013, les établissements toulousains publient en commun un carnet de recherche consacré aux AO, Toul'AO. ${ }^{20}$ En 2016, ils ont organisé un colloque intitulé Open Access et évaluation de la recherche : vers un nouvel écosystème? ${ }^{21}$

Le site toulousain semble cependant être une heureuse exception en matière de coopération inter-établissements en faveur de l'OA.

\section{Rennes 1}

L'Université de Rennes 1 propose une panoplie complète en matière d'OA : un portail dans HAL ouvert en 2012, un mandat d'accès ouvert depuis 2017 et un portail très riche, sur l'Open Access, animé par le SCD. On y trouve à la fois des explications générales sur l'OA, des précisions sur la politique OA de l'établissement et des informations pratiques destinées à faciliter le dépôt dans HAL par les chercheurs, que le SCD accompagne dans leur démarche. ${ }^{22}$

\section{Lille}

L'Université de Lille édite un portail de services aux chercheurs, animé par son SCD, Lilliad. ${ }^{23}$ Il comprend des explications sur le droit d'auteur, l'OA et les données de la recherche. Un outil en ligne, dénommé Willo, permet aux auteurs de déterminer rapidement leurs droits et obligations en matière de publication en OA. ${ }^{24}$

\section{CIRAD}

Le Centre de coopération internationale en recherche agronomique pour le développement (CIRAD) offre un très riche contenu d'aide à la publication sur son site web. La partie « Publier et diffuser » aborde en détail les questions d'OA. ${ }^{25}$

\footnotetext{
20. Toul'AO / Toulouse Archives Ouvertes. URL : http://openarchiv.hypotheses . org/ (visité le $19 / 01 / 2018)$.

21. Open Access et Evaluation de la Recherche. Oct. 2016. URL : https://openeval2016.sciencesco nf.org/ (visité le 19/02/2018).

22. Open Access / Université de Rennes 1. URL : https://openaccess.univ-rennes1.fr/ (visité le 28/03/2018).

23. Chercheur/LILLIAD. URL : https://lilliad.univ-lille.fr/chercheur (visité le 28/03/2018).

24. WillO. URL : https://decadoc.typeform. com/to/W2ZZMV (visité le 28/03/2018).

25. Publier et diffuser / Aide à la publication - Coopérer en information scientifique et technique Cirad. URL : https://coop-ist.cirad.fr/aide-a-la-publication/publier-et-diffuser (visité le 28/03/2018).
} 


\subsubsection{Financement de l'OA}

Début 2018, l'Université Toulouse Jean-Jaurès et l'Université de Lorraine ont communiqué sur leur politique de financement de l'OA. Dans les deux cas, cette politique consiste à se désabonner de ressources numériques commerciales considérées comme trop chères par rapport à leur usage et à réaffecter une partie des économies ainsi réalisées au financement d'actions de soutien à des modèles alternatifs de publication scientifique en OA. ${ }^{26}$

\subsection{Manifestations à l'occasion de l'Open Access Week}

L'Open Access Week est un évènement mondial de communication sur le libre accès, initié en 2007. Depuis 2009, l'Open Access Week se déroule chaque chaque année sur la dernière semaine complète du mois d'octobre. ${ }^{27}$

Afin de tenter d'estimer l'implication des acteurs français dans l'Open Access Week, un recensement des initiatives françaises à l'occasion de l'Open Access Week 2017 a été effectué.

\subsubsection{Une information dispersée}

La page Open Access week du site Open Access France mentionne : « Couperin centralise les évènements organisés en France lors des semaines mondiales de l'open access ». Pourtant, pendant l'OA week 2017, aucun événement n'était indiqué. Seuls quelques événements de 2015 et 2016 étaient mentionnés ${ }^{28}$.

Le site de l'International Open Access Week ne mentionne que trois événements en France en $2017^{29}$.

Le groupe Open Access France, sur le site web de l'Open Access Week, semble avoir vécu entre 2010 et 2015 et être en sommeil depuis ${ }^{30}$. Un message du 11 septembre 2015 renvoie vers un nouveau site dédié à l'Open Access Week en France ${ }^{31}$. C'est ce site qui paraît être le recueil le plus complet des manifestations françaises de l'Open Access Week depuis 2014 .

Enfin, certains établissements ont organisé des manifestations dans le cadre de l'Open Access Week sans pour autant le signaler sur Oaweekfrance ${ }^{32}$. Leur recensement serait trop long à effectuer dans le cadre du présent rapport.

26. Françoise Gouzi. Comment financer l'Open Access? Un exemple local. Toul'AO. 12 mar. 2018. URL : https : //openarchiv. hypotheses.org/4461 (visité le 16/04/2018); L'Université de Lorraine s'engage en faveur de la science ouverte / Factuel. 19 avr. 2018. URL : http://factuel.univ-lorraine. fr/node/8472 (visité le 23/04/2018).

27. Open Access Week: About. URL : http://openaccessweek.org/page/about (visité le 02/05/2018).

28. http://openaccess.couperin.org/category/nos_evenements/oaweek/ [consulté le 27 octobre 2017]

29. http://openaccessweek.org/events/event/listByLocation?location=France [consulté le 27 octobre 2017]

30. http://www.openaccessweek.org/group/openaccessfrance [consulté le 27 octobre 2017]

31. http://www. oaweekfrance.org/ [consulté le 27 octobre 2017]

32. Exemples:

http://www.scd.univ-smb.fr/index.php/11748 [consulté le 27 octobre 2017]

https://bibliotheques . univ-grenoble-alpes.fr/atelier-hal-le-nouveau-depot-simplifie$267570 . \mathrm{kj}$ sp?RH=2413488543318050 [consulté le 27 octobre 2017] 


\subsubsection{Les manifestations 2017}

Les événements recensés par le site OA Week France s'étalent du 11 octobre au 13 novembre, donc au-delà des dates officielles de l'OA Week, qui allaient 23 au 29 octobre.

Il n'est pas simple d'avoir une vue exhaustive. Un clic sur « programme ${ }^{33}$ permet d'obtenir une liste d'événements sous forme de deux pages HTML de tableaux. Cette liste est cependant incomplète, car un clic sur chacune des dates du calendrier permet d'afficher des éléments supplémentaires ${ }^{34}$.

Les catégories de manifestation (atelier, conférence, stand, table ronde) sont parfois trompeuses. Ainsi, plusieurs «stands » comprennent aussi des ateliers d'aide au dépôt. De plus, quelques manifestations n'ont pas été catégorisées par leurs organisateurs.

Le tableau des manifestions 2017 joint en annexe a donc été constitué en croisant les données du programme général et les données par date et complété le cas échéant par des détails intéressants lors qu'ils figuraient dans la notice détaillée de chaque événement.

On ne sera pas surpris de la place occupées par l'Ile-de-France, le Grand Est ou l'Occitanie, qui sont de grandes régions universitaires. En revanche, on s'étonnera de la faible représentation d'Auvergne-Rhône-Alpes ou de la Nouvelle Aquitaine. Enfin, on constatera l'absence totale de certaines régions comme le Centre-Val-de-Loire, la Corse ou la Normandie. Il peut cependant autant s'agir d'une absence de manifestations que de l'absence d'annonce de manifestations sur le site OA Week France.

Les bibliothèques et services d'IST sont dominants parmi les initiateurs des manifestions recensées.

On remarquera particulièrement l'Ecole Normale Supérieure, qui a organisé quatre événements à elle seule et les deux établissements présents sur plusieurs sites, l'Ensam et l'Inria, qui ont veillé à organiser des actions dans chacune de leurs implantations.

\subsection{Journées d'étude}

Sans prétendre à l'exhaustivité, quelques journées étude organisées en France ces dernières années ont été repérées.

La palme de l'antériorité et de la constance revient à Couperin, qui, de 2005 à 2018, a organisé six manifestions. D'abord limité à une journée et focalisé sur les archives ouvertes, le format s'est progressivement étendu à trois jours, le thème passant des archives ouvertes à l'open access, puis de l'open access à la science ouverte. ${ }^{35}$

En octobre 2016, le réseau « Archives ouvertes Toulouse » a consacré deux journées à l'étude du thème Open Access et évaluation de la recherche : vers un nouvel écosystème?. ${ }^{36}$

Dans le cadre de l'Open Access Week 2017, l'Université de Lyon a organisé une journée sur le thème de L'open access révélateur de synergies locales. ${ }^{37}$

\footnotetext{
33. http://www. oaweekfrance.org/programme/ [consulté le 27 octobre 2017]

34. Par exemple http://www.oaweekfrance.org/programme/2017-10-24/ [consulté le 27 octobre 2017]

35. Journées OA Couperin. 2005. URL : http://openaccess.couperin.org/category/nos_evenemen ts/joa/ (visité le 30/04/2018).

36. Open Access et Evaluation de la Recherche, op. cit.

37. L'open access révélateur de synergies locales. 24 oct. 2017. URL : https://oawlyon2017.sciences conf.org/ (visité le 19/02/2018).
} 
En mars 2018, l'Urfist de Bordeaux a réuni chercheurs et professionnels de l'IST pour réfléchir sur Le peer review à l'ère de l'open science : enjeux et évolutions. ${ }^{38}$

En avril 2018, ISSN France et l'Unesco ont organisé une conférence internationale à Paris, intitulée Une perspective internationale sur le libre accès dans la communication scientifique : succès et défis. ${ }^{39}$

La journée d'étude de l'Université de Lyon à l'occasion de l'Open Access Week 2018 a eu pour thème Bibliothèques et chercheurs : la science ouverte en commun. ${ }^{40}$

En novembre 2018, le groupe Open Access Languedoc Roussillon a consacré une journée à Être vu pour être lu l'open access pour renforcer votre identité numérique de chercheur, ${ }^{41}$ tandis que l'INSA de Lyon proposait de réfléchir sur la Visibilité des archives ouvertes. ${ }^{42}$

Enfin, en décembre 2018, les premières Journées Nationales de la Science Ouverte: de la stratégie à l'action se tenaient à Paris, à l'initiative du Comité pour la science ouverte (CoSO). ${ }^{43}$

38. Le peer review à l'ère de l'open science : enjeux et évolutions. 27 mar. 2018. URL : http:// weburfist.univ-bordeaux.fr/le-peer-review-a-lere-de-lopen-science-enjeux-et-evolutions/ (visité le 16/04/2018).

39. An International Perspective on Open Access in Scholarly Communication : Achievements and Challenges / Une perspective internationale sur le libre accès dans la communication scientifique : succès et défis. 26 avr. 2018. URL : http: //www . issn . org/wp-content/uploads/2018/04/ISSN_UNESCO_ PROGRAMME_CONFERENCE_20180426-1.pdf (visité le 30/04/2018).

40. Bibliothèques et chercheurs : la science ouverte en commun. URL : https://oawlyon2018.scienc esconf .org/ (visité le 10/10/2018).

41. Journée d'étude Open Access LR "Être vu pour être lu" - Open Access LR. URL : https://www. open-access.fr/actualites/41-journee-d-etude-open-access-lr-etre-vu-pour-etre-lu (visité le 06/12/2018).

42. Visibilite des archives ouvertes. URL : https ://visiarchives.sciencesconf .org/ (visité le 06/12/2018).

43. Les premières journées de la Science Ouverte. URL : https://jnso2018.sciencesconf .org/ (visité le $16 / 10 / 2018)$. 


\section{Chapitre 3}

\section{Obstacles}

La tentative d'état des lieux de l'OA dans le monde de l'ESR français, dressée au chapitre précédent, fait apparaître un contraste. D'un côté, on trouve quelques grandes infrastructures publiques, comme HAL, Persée ou OpenEdition, qui structurent fortement le paysage. De l'autre, un foisonnement d'initiatives montre que les bibliothèques sont très largement à l'origine du développement de l'OA. Ces initiatives sont cependant très dispersées, avec une quasi absence de mutualisation autour d'outils communs.

Le développement de l'OA se heurte à de nombreux obstacles invoqués par les chercheurs. Il sont généralement dus à leur méconnaissance du fonctionnement de l'OA. Afin de fournir aux bibliothécaires des outils pour lever ces obstacles, ce chapitre est un argumentaire, rédigé sous forme de foire aux questions (FAQ). Ce chapitre a été rédigé à partir de mon expérience personnelle, du site de Rennes dédié à l'OA et du carnet de recherche des acteurs toulousains de l'OA, dans lesquels on trouvera de nombreux élements de réponse aux questions des chercheurs. ${ }^{1}$ Les concepts évoqués sont développés dans le premier chapitre du présent rapport.

\subsection{C'est quoi, les archives ouvertes?}

Aussi surprenant que cela puisse paraître, il existe encore des enseignants chercheurs qui n'ont jamais entendu des archives ouvertes, ou qui ne savent pas vraiment ce que c'est.

Ainsi, j'ai longtemps fait face à l'absence de réponse à mes messages de promotion des archives ouvertes du directeur d'un laboratoire de SHS. Le rencontrant un jour dans des circonstances fortuites, je lui ai rappelé qu'il n'avait répondu à aucun de mes messages. Surpris, il me dit : «Je croyais que ce message ne concernait que les sciences dures ». Après quelques minutes de discussion, il comprit que sa structure de recherche était également concernée et me promit de me trouver un interlocuteur. Le lendemain, un membre de son équipe prit contact avec moi, début d'une collaboration efficace entre le SCD et le laboratoire, aboutissant au dépôt dans HAL de plusieurs publications.

Cette anecdote montre que si les bibliothécaires considèrent largement l'OA comme une évidence, c'est loin d'être la cas des chercheurs. Il convient donc de prendre le temps nécessaire pour leur expliquer de quoi il s'agit et, autant que possible de manière individualisée.

1. Open Access / Université de Rennes 1, op. cit.; Toul'AO / Toulouse Archives Ouvertes, op. cit. 


\subsection{Y ai-je intérêt?}

\subsubsection{Je ne veux pas divulguer ma production sans contrôle}

Comme son nom l'indique, une publication consiste à rendre publique les résultats d'une recherche. Mais un chercheur peut souhaiter choisir le moment où ses travaux seront visibles en OA, par exemple pour avoir le temps de préparer un livre sur le même sujet.

Lorsqu'un chercheur dépose une publication dans HAL, il peut la rendre visible immédiatement ou après un délai, appelé embargo, dont il peut lui-même fixer la durée.

De plus, le dépôt en archive ouverte constitue un bon moyen de prouver la date d'antériorité et la paternité d'un article, attestés par un numéro d'enregistrement unique. Les documents en OA peuvent être facilement intégrés dans les bases de connaissance des logiciels de détection de similitude. Pour toutes ces raisons, le dépôt en archive ouverte permet de lutter contre le plagiat.

\subsubsection{Est-ce bon pour ma carrière?}

Plusieurs études ont montré que les articles disponibles en OA sont plus cités que les autres. $^{2}$

En revanche, le dépôt et la publication en OA sont encore peu pris en compte par les instances d'évaluation. Elles ne sont cependant aucunement nuisibles.

\subsubsection{La publication en OA n'offre pas la garantie de qualité de l'édi- tion commerciale}

Le mode de diffusion n'est pas corrélé avec la qualité des articles publiés. Il y a de nombreuses revues avec comité de lecture en OA, tout comme il y a des revues peu scrupuleuses diffusées sur abonnement.

\subsubsection{HAL est encore un de ces machins franco-français : je préfère déposer mes publications sur Academia ou ResearchGate pour avoir une vraie visibilité internationale}

Contrairement à ce que pourrait laisser croire son origine française, HAL est très bien indexé par les moteurs de recherche dans le monde entier. Une recherche avec Google Scholar, qui est le moteur de recherche favori des chercheurs, fait remonter les publications déposées dans HAL parmi les premiers résultats. HAL est également référencé par d'autres moteurs de recherche et bases de données ${ }^{3}$

Academia et ResearchGate sont des réseaux sociaux académiques (RSA) et non de dépôts d'archives ouvertes. La réponse à l'objection « Je trouve déjà ce que je cherche sur Academia ou ResearchGate », plus loin dans le présent chapitre, explique pourquoi un RSA ne peut pas tenir lieu de dépôt d'archives ouvertes.

2. 7 bonnes raisons de déposer dans l'archive ouverte HAL. 2 mai 2016. URL : https://openaccess.u niv-rennes1.fr/7-bonnes-raisons-de-deposer-dans-larchive-ouverte-hal (visité le 04/04/2018).

3. Visibilité des dépôts HAL : moissonnage, signalement - HAL Documentation. URL : https : // doc . archives-ouvertes . fr/guide_utilisateurs / visibilite-des-depots - hal-moissonnagesignalement/ (visité le 04/04/2018). 


\subsection{Ai-je le droit?}

\subsubsection{La loi permet le dépôt en archive ouverte}

L'article 30 de la loi no 2016-1321 du 7 octobre 2016 pour une République numérique permet le dépôt en archive ouverte des écrits scientifiques financé au moins pour moitié sur fonds publics français ou européens. C'est donc le cas des articles des chercheurs des universités et organismes de recherche publique. Cet article a été intégré au Code de la Recherche (article L533-4) :

I.-Lorsqu'un écrit scientifique issu d'une activité de recherche financée au moins pour moitié par des dotations de l'Etat, des collectivités territoriales ou des établissements publics, par des subventions d'agences de financement nationales ou par des fonds de l'Union européenne est publié dans un périodique paraissant au moins une fois par an, son auteur dispose, même après avoir accordé des droits exclusifs à un éditeur, du droit de mettre à disposition gratuitement dans un format ouvert, par voie numérique, sous réserve de l'accord des éventuels coauteurs, la version finale de son manuscrit acceptée pour publication, dès lors que l'éditeur met lui-même celle-ci gratuitement à disposition par voie numérique ou, à défaut, à l'expiration d'un délai courant à compter de la date de la première publication. Ce délai est au maximum de six mois pour une publication dans le domaine des sciences, de la technique et de la médecine et de douze mois dans celui des sciences humaines et sociales. La version mise à disposition en application du premier alinéa ne peut faire l'objet d'une exploitation dans le cadre d'une activité d'édition à caractère commercial.

$[\ldots]$

IV.-Les dispositions du présent article sont d'ordre public et toute clause contraire à celles-ci est réputée non écrite.

Aucun texte n'a pour l'instant tranché le cas des publications antérieures à la promulgation de la loi. Un certain consensus (Conseil scientifique du CNRS, Couperin) penche en faveur d'une application de la loi à ces publications. De plus, aucune action en justice n'a été intentée par des éditeurs pour le dépôt en archive ouverte d'une de ces publications

\subsubsection{Des outils pour déterminer le droit}

Plusieurs outils permettent aux chercheur et aux professionnels de l'IST qui l'accompagnent de déterminer sa situation juridique en vue d'un dépôt en archive ouverte.

La FAQ de Couperin, consacrée à la Loi pour une République numérique, répond à un grand nombre de questions que peut se poser un chercheur souhaitant déposer une publication. ${ }^{4}$

SHERPA/RoMEO ${ }^{5}$ et Héloïse ${ }^{6}$ donnent accès aux politiques des éditeurs en matière de dépôt en archive ouverte. Lorsque la politique de l'éditeur est plus restrictive

4. FAQ sur la loi République numérique - Open Access France. URL : http://openaccess.couperin. org/category/faq/ (visité le 05/04/2018).

5. SHERPA/RoMEO - Publisher copyright policies \& self-archiving. URL : http://www.sherpa.ac. $\mathrm{uk} / \mathrm{romeo} /$ index.php (visité le 19/02/2018).

6. Héloïse - Politiques des éditeurs en matière de dépôt des articles. URL : https://heloise.ccsd. cnrs.fr/ (visité le 19/02/2018). 
que les dispositions de la Loi pour une République numérique, c'est la loi qui s'applique de plein droit.

Willo est un outil développé par le SCD de l'Université de Lille, qui permet de déterminer le droit de dépôt s'appliquant à une publication en répondant à une suite de questions. ${ }^{7}$

Enfin, Dissemin, développé par une équipe de l'ENS, se présente comme « une plateforme web qui récolte des métadonnées à partir de différentes sources et qui détermine la disponibilité du texte intégral des publications des chercheurs. Il a été conçu pour encourager l'usage des dépôts ». Dissemin permet à un chercheur d'avoir rapidement une vue sur le droit applicable à l'ensemble de ses travaux déjà en ligne, y compris en accès payant. ${ }^{8}$

\subsection{C'est trop compliqué / Je n'ai pas le temps}

Le dépôt en archive ouverte demande qu'on y consacre un peu de temps, mais généralement moins qu'on l'imagine. L'interface de dépôt de HAL s'est fortement simplifiée à l'automne 2017. Une procédure automatique parcourt le fichier PDF pour en extraire les métadonnées (titre, auteurs, affiliations, résumé...), qu'il suffit de vérifier.

Le temps des chercheurs est précieux. Il revient donc à l'établissement d'organiser un système de dépôt adapté aux souhaits et aux possibilités de chacun :

- dépôt par le chercheur lui-même

- dépôt par un personnel ITRF du laboratoire

- dépôt par un personnel du SCD

Dans les deux premiers cas, le SCD devra pouvoir intervenir en soutien, à la demande du chercheur ou du laboratoire. Et dans tous les cas, le SCD procédera régulièrement à l'harmonisation des métadonnées et au contrôle de la qualité de l'archive ouverte de l'établissement.

\subsection{Je trouve déjà ce que je cherche ailleurs}

Certains chercheurs ont le sentiment de disposer d'alternatives plus simples que les archives ouvertes. Il convient alors de leur démontrer qu'elles sont peu pérennes et parfois même illégales.

\subsubsection{Je trouve déjà presque tout ce que je cherche sur gratuitement sur Internet}

Si c'est le cas, c'est probablement parce que l'établissement du chercheur est abonné à de nombreux bouquets d'éditeurs commerciaux et qu'il permet d'y accéder par la reconnaissance d'adresse IP. Mais le coût de ces abonnements a augmenté beaucoup plus vite que l'inflation (de 5 à $10 \%$ par an) et il est très probable que l'établissement sera conduit à renoncer à plusieurs abonnements dans les prochaines années, s'il n'a pas déjà commencé à le faire.

7. WillO, op. cit.

8. Dissemin. URL : https://dissem.in/ (visité le 05/04/2018). 


\subsubsection{Je trouve déjà ce que je cherche sur Academia / ResearchGate}

Bien qu'il permettent de déposer facilement des publications, Academia.edu ${ }^{9}$ et ResearchGate $^{10}$ sont des réseaux sociaux académiques (RSA) et non de dépôts d'archives ouvertes.

Les RSA recueillent des données sur leurs utilisateurs (domaine d'activité, réseau relationnel, publications), en l'occurrence les chercheurs, qui cèdent aux RSA de nombreux droit de manière irrévocable, souvent en violation des contrats passés avec leurs éditeurs. ${ }^{11}$

Les RSA ne garantissent pas la pérennité de la présence en ligne des documents déposés par le chercheur. Ainsi, plusieurs articles ont été dépubliés d'Academia et de ResearchGate, à la demande des éditeurs.

Les RSA sont des startups. Il faut garder à l'esprit que la stratégie habituelle d'une startup consiste à prendre de la valeur pour se faire racheter très cher par un grand groupe après quelques années d'existence. Mendeley, outil combinant des fonctions de gestion bibliographique, de réseau social et de partage de PDF entre chercheurs, fondé en 2007 a ainsi été racheté par Elsevier en $2013^{12}$

Aline Bouchard, conservateur à l'Urfist de Paris, a publié en novembre 2016 un document de comparaison entre les archives ouvertes et les RSA. Très détaillé, il comprend un tableau synthétique pédagogique et facile à utiliser lors du dialogue avec les chercheurs. On pourra ajouter que le dépôt dans HAL s'est considérablement simplifié depuis l'automne 2017 et que HAL est très bien référencé par Google Scholar. ${ }^{13}$

Les RSA doivent donc être utilisés par les chercheurs pour ce qu'ils sont : des réseaux sociaux. La bonne pratique consiste donc à déposer leurs articles sur HAL ou sur l'AOI de leur établissement et, s'ils souhaitent utiliser un RSA, d'y mettre des liens vers l'archive ouverte qui conserve leurs travaux. ${ }^{14}$

\subsubsection{Unpaywall / Lazy Scholar / Open Access Button / Kopernio me permet de trouver gratuitement ce que je cherche}

Unpaywall, Lazy Scholar, Open Access Button et Kopernio sont des outil web qui permettent de savoir s'il existe une version gratuite d'une publication payante qu'on cherche à lire et, le cas échéant d'y accéder. Les chercheurs auraient donc tort de se priver de tels outils. ${ }^{15}$

Il convient cependant de les sensibiliser sur le fait qu'ils ne donnent accès qu'à des publications déjà disponibles en OA. Pour qu'ils puissent fonctionner au bénéfice de tous, il faut que chacun fasse l'effort de publier ou de déposer en OA.

9. Academia.edu, op. cit.

10. ResearchGate, op. cit.

11. Christophe Benech. Protection et propriété des données sur Academia.edu et ResearchGate. 14 mar. 2014. URL : https://archeorient.hypotheses.org/2554 (visité le 31/03/2018).

12. Aline Bouchard. Pour une utilisation critique des réseaux sociaux académiques. 14 fév. 2014. URL : https://urfistinfo.hypotheses.org/2596 (visité le 31/03/2018).

13. Aline Bouchard. Éléments de comparaison archives ouvertes (HAL) et réseaux sociaux académiques (Academia, Research Gate). Nov. 2016. URL : http://urfist.chartes.psl.eu/sites/default/ files/ab/Bouchard_Comparaison_AO_RSX_112016.pdf (visité le 30/03/2018), p. 2.

14. Françoise GouzI. Academia.edu, ResearchGate...̀̀ utiliser avec modération! Toul'AO. 29 jan. 2015. URL : http://openarchiv.hypotheses.org/2582 (visité le 19/02/2018).

15. Des extensions de navigateurs pour repérer les articles en libre-accès - BU Lyon 1 - Université Lyon 1, op. cit. 


\subsubsection{Il suffit de demander sur Twitter avec le hastag \#IcanHazPDF}

On peut l'envisager comme une solution de dépannage ponctuel, mais il faut avoir conscience qu'elle est illégale. Elle est de plus très aléatoire. Un chercheur ne peut donc pas s'en contenter pour bâtir sa stratégie d'accès à la documentation.

\subsubsection{Il y a tout sur SciHub et LibGen}

SciHub et Library Genesis (LibGen) sont des bases de données de publications scientifiques piratées sur les sites des grands éditeurs commerciaux. En raison de leur richesse de contenu et de leur facilité d'utilisation, elles sont très utilisées, tant dans les pays pauvres que dans les pays riches.

Certains chercheurs voient dans SciHub et LibGen une nécessaire révolte visant à l'émancipation des publications scientifiques face à l'oligopole des grands éditeurs commerciaux. SciHub et LibGen n'en sont pas moins illégales et ont fait l'objet de condamnations en justice à la demande des éditeurs. Leur accès est aléatoire et elles doivent régulièrement changer d'URL au fur et à mesure des condamnations.

Chaque chercheur pourra, en son âme et conscience, décider ou non de les utiliser. Cependant, aucun ne pourra en faire une source avouée sans prendre de risque juridique pour lui et pour son institution de rattachement.

\subsubsection{Je publie déjà dans des revues en OA}

Il s'agit évidemment d'une situation favorable : le chercheur sait ce qu'est l'OA et sait aussi que les revues dans lesquelles il publie sont en OA. Il convient cependant d'attirer son attention sur sur deux cas particuliers, les revues hybrides et les revues prédatrices.

\section{Les revues hybrides}

Les revues hybrides sont principalement financées par les abonnements. Cependant, elles offrent aussi la possibilité de publier des articles en OA, financés par des APC. La formule peut paraître séduisante de prime abord. En fait, elle revient généralement à augmenter les recettes de l'éditeur, sans pour autant faire baisser les coûts pour les chercheurs et leurs institutions. Les revues hybrides sont donc à éviter.

\section{Les revues prédatrices}

Une revue prédatrice est une revue qui, profitant de l'intérêt de la communauté scientifique pour l'OA, a été créée pour encaisser des APC sans rendre de réel service au chercheur.

Les revues prédatrices ciblent particulièrement les nouveaux docteurs, en leur promettant un publication rapide à faible coût. Leur titre ressemble souvent à celui d'une revue réputée, avec de légères différences. Au minimum, le chercheur aura dépensé inutilement le coût des APC. De plus, une publication dans une revue prédatrice pourra nuire à sa réputation et à celles de son institution.

Il convient donc de fuir les revues prédatrices. Des guides permettent de les repérer avant de signer trop rapidement un contrat d'édition. ${ }^{16}$

16. Fovet-RAвот, Eviter les éditeurs prédateurs, op. cit.; Rennes 1, Les revues prédatrices, op. cit.; Stop Predatory Journals, op. cit. 


\subsection{Je ne sais pas où déposer mes publications}

Dans le contexte français, trois solutions s'offrent au chercheur pour déposer ses publications dans une archive ouverte. Il s'agit de HAL, de l'archive institutionnelle de son établissement ou de l'archive thématique de sa discipline. Ces solutions ne sont pas exclusives l'une de l'autre et il existe des passerelles entre elles.

\subsubsection{HAL}

HAL est de loin le dépôt d'archives ouvertes le plus employé en France. Développé depuis 2001 par le CCSD du CNRS, il a démontré se qualités en matière de pérennité et d'évolutivité.

HAL est une grande archive ouverte pluridisciplinaire unique, qui peut se présenter sous plusieurs interfaces spécifiques, appelés portails. Les portails institutionnels permettent de rendre visibles les publications des chercheurs d'une institution. Ce sont en quelque sorte des AOI au sein de HAL. Il existe aussi quelques portails thématiques, le principal étant HAL-SHS. Enfin, on peut créer des collections, surtout utiles pour présenter de façon personnalisées tout ou partie des productions d'un laboratoire. Il faut toujours garder en tête que les portails et collections ne sont que des vues partielles sur HAL, qui est une base de données unique. Un document déposé dans HAL apparaîtra donc automatiquement dans le portail institutionnel de l'établissement du chercheur et réciproquement.

Enfin, HAL permet au chercheur de créer son identifiant, appelé IdHAL, auquel il peut faire correspondre son identifiant ORCID. Une fois cette étape franchie, le chercheur peut générer facilement un $\mathrm{CV}$ avec la liste de ses publications.

\subsubsection{Archive ouverte institutionnelle}

Du fait du développement de HAL, peu d'établissements français ont créé leur propre archive ouverte institutionnelle (AOI). Cependant, lorsqu'elle existe, il est bien sûr souhaitable de l'utiliser.

Les AOI sont souvent moissonnées par HAL. Le cas échéant, SCD de l'établissement pourra le confirmer.

\subsubsection{Archive ouverte thématique}

Le dépôt archive ouverte thématique est surtout pratiqué par les physiciens, qui utilisent massivement arXiv. arXiv est aussi ouverte aux mathématiques, informatique, génie électrique, biologie et finance quantitatives, statistiques et économie.

Lorsqu'un chercheur utilise HAL, il peut demander à ce que son article soit automatiquement déposé dans arXiv si sa discipline est concernée. ${ }^{17}$

17. Transférer le dépôt vers arXiv-HAL Documentation. URL : https://doc.archives-ouvertes . $\mathrm{fr} /$ deposer/transfert-hal-arxiv/ (visité le 04/04/2018). 


\subsection{J'aimerais publier dans une revue en OA, mais je ne sais pas comment m'y prendre}

En France, la principale plateforme de publication de revues en OA est OpenEdition. Il existe aussi Persée, dont la vocation est la numérisation et la diffusion en OA d'archives de revues imprimées.

Quelques universités ont créé leur propre plateforme de revues en OA. Si un groupe de chercheurs est motivé par cette démarche, il est souhaitable que le SCD les soutienne pour faire de même dans leur établissement.

Enfin, des communautés disciplinaires se sont organisées au sein de la Fair Open Access Alliance (FOAA). Elles ont quitté leur éditeur commercial historique, pour créer de nouvelles revues avec le même comité éditorial. Là encore, si des chercheurs sont motivés pour s'engager collectivement dans cette voie, ils doivent pouvoir compter sur l'assistance des bibliothécaires de leurs établissements. 


\section{Chapitre 4}

\section{Propositions}

Face aux obstacles évoqués dans le chapitre précédent, il est maintenant nécessaire de faire des propositions pour les aplanir. Celles-ci concernent la communication sur le droit, l'évaluation des chercheurs, le rôle des établissements d'ESR, de leurs SCD et de HAL. Enfin, la création d'une plateforme fédératrice de l'action des bibliothèques pour le développement de l'OA permettrait de mutualiser les efforts déjà entrepris.

\subsection{Communiquer largement sur les objectifs et possibili- tés de diffusion des travaux de la recherche}

Comme on l'a vu précédemment, la méconnaissance de l'OA est un obstacle important à son développement. Il importe donc de faire connaître largement son cadre juridique et les initiatives académiques en sa faveur.

\subsubsection{Le code de la recherche}

Le code de la recherche mentionne l'OA à deux reprises. L'article L112-1 traite des objectifs de la recherche publique, dont font partie le partage et la diffusion des connaissances et des données scientifique, en priorité à des formats libres d'accès. L'article L533-4, créé par l'article 30 de la loi no 2016-2016-1321 du 7 octobre 2016 pour une République numérique, dite Loi Lemaire, donne aux chercheurs le droit de déposer leurs écrits scientifiques en archive ouverte ${ }^{1}$ :

\section{Article L112-1}

La recherche publique a pour objectifs :

a) Le développement et le progrès de la recherche dans tous les domaines de la connaissance;

b) La valorisation des résultats de la recherche au service de la société, qui s'appuie sur l'innovation et le transfert de technologie;

c) Le partage et la diffusion des connaissances scientifiques en donnant priorité aux formats libres d'accès ;

c bis) Le développement d'une capacité d'expertise et d'appui aux associations et fondations, reconnues d'utilité publique, et aux politiques publiques

1. Les mises en gras ont été ajoutées pour les besoins du présent rapport. 
menées pour répondre aux défis sociétaux, aux besoins sociaux, économiques et du développement durable ;

d) La formation à la recherche et par la recherche;

e) L'organisation de l'accès libre aux données scientifiques.

Les établissements publics de recherche et les établissements d'enseignement supérieur favorisent le développement des travaux de coopération avec les associations et fondations, reconnues d'utilité publique. Ils participent à la promotion de la recherche participative et au développement des capacités d'innovation technologique et sociale de la Nation. Ces coopérations s'exercent dans le respect de l'indépendance des chercheurs et, en l'absence de clauses contraires, dans un but non lucratif. Les travaux de recherche menés dans le cadre de ces coopérations sont, en l'absence de clauses contraires, rendus publics et accessibles.

\section{Article L533-4}

I.-Lorsqu'un écrit scientifique issu d'une activité de recherche financée au moins pour moitié par des dotations de l'Etat, des collectivités territoriales ou des établissements publics, par des subventions d'agences de financement nationales ou par des fonds de l'Union européenne est publié dans un périodique paraissant au moins une fois par an, son auteur dispose, même après avoir accordé des droits exclusifs à un éditeur, du droit de mettre à disposition gratuitement dans un format ouvert, par voie numérique, sous réserve de l'accord des éventuels coauteurs, la version finale de son manuscrit acceptée pour publication, dès lors que l'éditeur met lui-même celle-ci gratuitement à disposition par voie numérique ou, à défaut, à l'expiration d'un délai courant à compter de la date de la première publication. Ce délai est au maximum de six mois pour une publication dans le domaine des sciences, de la technique et de la médecine et de douze mois dans celui des sciences humaines et sociales. La version mise à disposition en application du premier alinéa ne peut faire l'objet d'une exploitation dans le cadre d'une activité d'édition à caractère commercial.

II.-Dès lors que les données issues d'une activité de recherche financée au moins pour moitié par des dotations de l'Etat, des collectivités territoriales, des établissements publics, des subventions d'agences de financement nationales ou par des fonds de l'Union européenne ne sont pas protégées par un droit spécifique ou une réglementation particulière et qu'elles ont été rendues publiques par le chercheur, l'établissement ou l'organisme de recherche, leur réutilisation est libre.

III.-L'éditeur d'un écrit scientifique mentionné au I ne peut limiter la réutilisation des données de la recherche rendues publiques dans le cadre de sa publication.

IV.-Les dispositions du présent article sont d'ordre public et toute clause contraire à celles-ci est réputée non écrite. 


\subsubsection{Les recherches subventionnées H2020}

Le programme européen de financement de la recherche Horizon 2020, plus connu sous son abréviation H2020, court sur la période 2014-2020. Il comporte l'obligation d'assurer le libre accès aux publications issues des recherches auxquelles elle aura attribué un financement, même partiel, en Green ou en Gold OA. La publication en OA des données de la recherche n'est pas obligatoire, mais elle est recommandée. ${ }^{2}$

OpenAIRE est le projet, financé en quasi-totalité par H2020, pour promouvoir et faciliter la publication en OA des publications scientifiques et des données de la recherche. ${ }^{3}$ OpenAIRE peut être considéré comme la brique OA d'H2020. Sa FAQ est très complète. ${ }^{4}$ Un dépôt de données de la recherche, Zenodo a été créé dans le cadre d'OpenAIRE. ${ }^{5}$

\subsection{3 cOAlition $\mathrm{S}$ et le Plan S}

Le 4 septembre 2018, onze organismes nationaux de financement de la recherche, dont l'Agence nationale de la recherche (ANR), ont lancé la cOAlition $S$ pour la mise en oeuvre du Plan $S$. Les membres de la cOAlition $S$ s'engagent à prendre les dispositions nécessaires, pour atteindre l'objectif du plan : «A partir du 1er janvier 2020, les publications scientifiques issues de la recherche financée par des subventions publiques nationales ou européennes devront être publiées en accès ouvert dans des revues ou sur des plateformes Open Access conformes ». Outre son objectif, le Plan S énonce dix principes sur la protection du droit des auteurs, le développement des revues de qualité en OA, l'encadrement des APC, le développement des archive souvertes et le refus des revues hybrides. ${ }^{6}$

\subsubsection{Les initiatives académiques en faveur de l'OA}

Les initiatives académiques en faveur de l'OA, présentées dans le deuxième chapitre du présent rapport, sont assez peu connues.

Des campagnes nationales de communication régulières, notamment à l'occasion de l'Open Access Week, sur les établissements ayant adopté un mandat de libre accès et/ou créé des services autour de l'OA, seraient d'une grande utilité.

\subsection{Rendre la publication en OA incitative pour la carrière des chercheurs}

Aujourd'hui, un chercheur investi dans la publication ou le dépôt en OA l'est en raison de ses seules convictions. Il n'en retire aucun bénéfice en terme de carrière.

2. Le libre accès aux publications et aux données de recherche - Horizon 2020. URL : http://www. horizon2020.gouv.fr/cid82025/le-libre-acces-aux-publications-aux-donnees-recherche.html (visité le 06/04/2018).

3. Project factsheets / General Information. URL : https://www.openaire.eu/project-factsheets (visité le 06/04/2018).

4. OpenAIRE - FAQ. URL : https://www.openaire.eu/faq (visité le 06/04/2018).

5. Zenodo - Research. Shared. URL : https://www.zenodo.org/ (visité le 06/04/2018).

6. Science Europe - cOAlition S. URL : https ://www.scienceeurope.org/coalition-s/ (visité le 07/09/2018); Le (bon) plan S. 4 sept. 2018. URL : https://marlenescorner.net/2018/09/04/le-bonplan-s/ (visité le 07/09/2018). 


\subsubsection{Le Conseil national des universités (CNU)}

Le Conseil national des universités (CNU) est une instance qui se prononce sur « les mesures individuelles relatives à la qualification, au recrutement et à la carrière des professeurs des universités et des maîtres de conférences. Il procède au suivi de carrière des enseignants-chercheurs ». Le CNU est organisé en 57 sections, dont chacune correspond à une discipline. ${ }^{7}$ " Pour chaque section, les critères et les modalités d'appréciation des candidatures ainsi que les modalités de mise en œuvre du suivi de carrière des enseignants-chercheurs sont rendus publics $\gg .^{8}$

La recherche des chaînes de caractères « ouvert », « archive » et « open », permettant d'identifier rapidement la présence d'expressions comme « archive(s) ouverte(s) », « accès ouvert » ou « open access » dans le rapport d'activité le plus récent de chacune des sections et dans les conseils aux candidats à la promotion de grade, montre que la publication ou le dépôt en OA n'est mentionné explicitement comme critère d'évaluation que par la section 60 (Mécanique, génie mécanique, génie civil). La façon dont les revues prises en compte sont catégorisées laisse cependant entrevoir un manque de familiarité avec l'OA :

En ce qui concerne les articles, ils doivent être classés dans les catégories suivantes :

- Articles dans des revues internationales à comité de lecture reconnues,

- Articles dans des revues internationales à comité de lecture «special issues »,

- Articles dans des revues nationales à comité de lecture,

- Articles dans des revues « open $» .^{9}$

Une action de sensibilisation de chacune des sections du CNU s'avère donc indispensable.

\subsubsection{Le Haut Conseil de l'évaluation de la recherche et de l'enseigne- ment supérieur (HCERES)}

Le Haut Conseil de l'évaluation de la recherche et de l'enseignement supérieur (HCERES) est une autorité administrative indépendante qui a pour mission d'évaluer les établissements d'ESR, afin de les aider à améliorer leurs pratiques et d'éclairer les décideurs.

Lors des Journées Science Ouverte 2018, Michel Cosnard, président du HCERES, a indiqué que le principal critère d'évaluation de la recherche était la « qualité des produits et activités de la recherche », qui se déclinait en «a) production de connaissance / rayonnement académique; b) interactions avec l'environnement; c) implication dans la formation par la recherche ». Il a cité l'OA à deux reprises, mais toujours comme un critère d'évaluation secondaire : « Valeur ajoutée de la mise à disposition de la production des unités au moyen d'archives ouvertes » et « Possibilité d'utiliser les archives ouvertes

7. Liste des sections $C N U$ - Portail. URL : http://www.cpcnu.fr/listes-des-sections-cnu (visité le $11 / 04 / 2018)$.

8. Décret $n^{\circ}$ 92-70 du 16 janvier 1992 relatif au Conseil national des universités. 16 jan. 1992. URL : https : / / www . legifrance . gouv . fr/affichTexte . do ? cidTexte= JORFTEXT000000344860 (visité le 19/02/2018).

9. Section 60 CNU. Recommandations et critères pour les promotions en section 60. URL : http: //www.cpcnu.fr/web/section-60/conseils-generaux1 (visité le 11/04/2018). 
pour avoir accès à la production des unités de recherche sans contraindre les enseignantschercheurs et les chercheurs à faire un double dépôt. » Par ailleurs, le HCERES envisage d'ouvrir son propre portail HAL pour y déposer les rapports qu'il produit. ${ }^{10}$

Pour que le HCERES considère l'OA comme un critère important d'évaluation, il faudra sans doute que l'OA soit affirmé comme une politique nationale.

\subsubsection{L'Agence nationale de la recherche (ANR)}

L'Agence nationale de la recherche (ANR) est un opérateur de l'Etat. Agence de moyens, elle lance des appels à projets. Elle sélectionne et finance environ $15 \%$ des projets de recherche qui lui sont présentés.

L'ANR est sensible à l'OA et lui consacre une page sur son site web. ${ }^{11}$ L'ANR a signé avec plusieurs organismes de recherche une convention de partenariat en faveur des archives ouvertes et de la plateforme mutualisée HAL. ${ }^{12}$ Cependant, à la différence, du programme européen H2020, qui en a fait une obligation, l'ANR « recommande que toutes les publications consécutives aux projets qu'elle finance, soient déposées en texte intégral dans une archive ouverte, soit directement dans HAL soit par l'intermédiaire d'une archive institutionnelle locale $»{ }^{13}$

En septembre 2018, l'ANR a signé la Déclaration de San Francisco sur l'évaluation de la recherche (DORA). Cette déclaration recommande de considérer la qualité des publications du chercheur et non la notoriété des revues dans lesquelles il publie. ${ }^{14}$

Là encore, il manque l'affirmation d'une politique nationale pour passer de la recommandation à l'obligation.

\subsubsection{Les conférences d'établissements (CPU, CDEFI, CGE)}

La Conférence des présidents d'universités (CPU), la Conférence des directeurs des écoles françaises d'ingénieurs (CDEFI) et la Conférence des grandes écoles (CGE), sont des associations chargées de représenter les établissements d'enseignement supérieur. Ce ne sont pas des instances d'évaluation et elles n'ont pas de pouvoir décisionnaire applicable à leurs membres. Elles ont cependant un réel pouvoir d'influence sur les décisions prises par l'Etat.

A l'occasion des Journées Science Ouverte, en janvier 2018, les trois conférences ont pris une position commune pour :

— promouvoir le dépôt des publications en open access dans des entrepôts ouverts ;

- organiser la préservation et la valorisation des données de la recherche et continuer à prendre la mesure des besoins en termes de fouille de données dans l'attente de la directive européenne sur le copyright;

\footnotetext{
10. Michel Cosnard. «L'évaluation au Hcéres ». JSO 2018. Paris. URL : https://jso2018. sciences conf.org/data/pages/Michel_Cosnard_2.pdf.

11. L'ANR et l'Open access. URL : http://www . agence-nationale-recherche.fr/missions-etorganisation/open-access/ (visité le 11/04/2018).

12. Convention de partenariat en faveur des archives ouvertes et de la plateforme mutualisée HAL. 2 avr. 2013. URL : http://cache.media.enseignementsup-recherche.gouv. fr/file/HAL/93/3/01_ Convention_HAL_246933.pdf (visité le 11/04/2018).

13. L'ANR et l'Open access, op. cit.

14. L'ANR, signataire de la Déclaration de San Francisco sur l'évaluation de la recherche. ANR. URL : http://www .agence-nationale-recherche.fr/informations/actualites/detail/lanr-signatairede-la-declaration-de-san-francisco-sur-levaluation-de-la-recherche/ (visité le 06/12/2018).
} 
- encourager les éditeurs qui innovent en proposant des dispositifs plus ouverts sur l'open access (EDP Sciences, CAIRN et Open Edition Premium);

- concevoir des dispositifs d'évaluation novateurs prenant en compte la communication scientifique ouverte ;

- encourager les établissements à définir une politique locale de science ouverte pour se doter, par exemple, d'une charte open access. ${ }^{15}$

Il reste maintenant aux établissements membres des conférences à se saisir de ces recommandations et encouragements.

\subsubsection{Le Comité pour la Science Ouverte (CoSO)}

Si le développement rapide et massif de l'OA est une réelle volonté politique, les conditions pour que les chercheurs y trouvent un intérêt pour leur carrière restent encore à créer. La transformation, en 2018, de la Bibliothèque scientifique numérique (BSN) en Comité pour la Science Ouverte (CoSO) est un pas dans cette direction :

Le CoSO a pour mission de définir une politique de science ouverte, d'en assurer le développement à l'échelle nationale et internationale, et d'en coordonner la mise en ouvre à l'échelle des établissements et des communautés scientifiques

$[\ldots]$

Il traite notamment de l'ouverture des publications et des données de la recherche ${ }^{16}$

Le CoSO est organisé en collèges et en groupes projets.

Les quatre collèges sont des commissions permanentes. Un est consacré aux publications et un autre aux données de la recherche.

Les groupes projet sont des commissions ad hoc. L'un d'entre eux est consacré à l'évaluation.

L'évaluation des chercheurs, avec ses conséquences sur l'évolution de leur carrière, l'évaluation de projets de recherche ainsi que l'évaluation des laboratoires s'appuient encore trop souvent sur des indicateurs bibliométriques inadaptés dont le trop célèbre et malfaisant facteur d'impact des revues. Cette pratique réductionniste constitue l'une des raisons majeures du quasimonopole de fait d'éditeurs capitalistiques et de la captation du temps? de l'expertise? d'un grand nombre de chercheurs dans certaines disciplines majeures.

$[\ldots]$

Dans ce contexte, le groupe-projet «Évaluation de la Recherche » doit s'appuyer sur des compétences et pratiques de différentes catégories d'acteurs : chercheurs, documentalistes, responsables de laboratoires, responsables d'institutions, chargés d'évaluation, HCERES, etc. Il ne peut prétendre apporter

15. Open access : une position commune de la CDEFI, la CGE et la CPU. URL : http://www.cdefi. $\mathrm{fr} / \mathrm{fr} / \mathrm{actualites/open-access-une-position-commune-de-la-cdefi-la-cge-et-la-cpu} \mathrm{(visité} \mathrm{le}$ 11/04/2018); Déclaration commune des trois conférences (CPU, CDEFI et CGE) au Journées Science ouverte 2018. Jan. 2018. URL : https://jso2018. sciencesconf .org/data/pages/20180122_JS02018_ TroisConferences_VF_2.pdf (visité le 11/04/2018).

16. AMI pour la constitution du Comité pour la Science Ouverte (CoSO), op. cit. 
des solutions clé en main, mais devra plutôt viser à favoriser le partage d'expériences et de bonnes pratiques et à sensibiliser les différents acteurs.

[...] [le groupe projet devra]

Proposer un mode d'organisation pérenne qui assurera un suivi à l'échelle nationale, voire européenne ${ }^{17}$

\subsubsection{La Commission européenne}

La Commission européenne a confié à un groupe présidé par Conor O'Carroll et Bernard Rentier la rédaction d'un apport sur l'évaluation des chercheurs. Ce rapport, publié en 2017, cherche à encourager, motiver et reconnaître les chercheurs qui s'engagent dans la science ouverte. Il invite à cesser de tenir compte du facteur, indicateur simple, mais qui ne mesure que la notoriété de la revue, et non la qualité des travaux du chercheur. A la place, il propose une matrice d'évaluation de carrière, appelée Open Science Career Assessment Matrix (OS-CAM), qui, avec 23 critères répartis en 6 catégories, tient compte de la complexité de l'activité du chercheur. La publication dans des revues en OA et le dépôt en archive ouverte y figurent explicitement comme des critères d'évaluation. ${ }^{18}$

On ne peut que recommander aux instances françaises chargées de l'évaluation et de la carrière des chercheurs d'étudier et de s'approprier ce rapport.

\subsection{Accélérer la prise en compte de l'OA par les établisse- ments}

\subsubsection{Lancer un appel à projet MESRI pour un label}

Les appels à projet et les labellisations font aujourd'hui partie des modes de management des organisations publiques. On pourrait envisager d'employer ce type de dispositif pour promouvoir l'OA.

Un label « Université promotrice de l'Open Access », qui se déclinerait sous une compacte « $\mathrm{U}+\mathrm{OA} »$ et une forme anglaise « OA frendly University », pourrait comprendre les éléments de labellisation suivant :

- équipe du SCD dédiée et formé à l'OA

- mandat de libre accès

- portail dans HAL ou AO institutionnelle moissonnée par HAL

— organisation d'évènements pendant l'OA week recensés sur le portail OA week France

Afin de tenir compte de la progressivité des efforts de l'établissement, le label pourrait comporter trois niveaux : un, deux ou trois cadenas ouverts, symboles de l'OA ou bien Bronze, Argent et Or si on préfère la métaphore sportive. Le premier niveau supposerait qu'au moins deux éléments de la labellisation soient opérationnels, chaque élément supplémentaire permettant d'atteindre un niveau supérieur.

Il ne s'agit bien sûr que d'une idée destinée à stimuler la réflexion et à provoquer l'émulation parmi les établissements. Si elle était retenue, un groupe de travail devrait en préciser les contours et les modalités de mise en oeuvre.

17. Ibid

18. O'CARroll et Rentier, Evaluation of Research, Careers fully acknowledging, Open Science practices, op. cit. 
4.3.2 Rendre la définition d'une politique d'OA obligatoire pour les établissements d'enseignement supérieur et de recherche et les organismes de recherche

Au-delà du label, qui a un rôle incitatif, une obligation de délibération politique sur l'OA est nécessaire pour que chaque établissement s'empare de la question. Cette obligation pourrait être inscrite dans les contrats pluriannuels, avec une date d'échéance impérative. Le CA de chaque établissement devrait a minima :

- décider de la création d'un portail dans HAL ou une AOI moissonnée par HAL ou transformer la collection en portail pour les établissements qui ont uniquement une collection;

- définir une politique écrite de dépôt en archive ouverte, précisant les obligations et dérogations adaptée aux disciplines et aux chercheurs de l'établissement.

\subsection{Renforcer les services communs de documentation (SCD) dans leur rôle d'acteur du développement de l'OA}

Globalement, les bibliothèques de l'ESR sont plus sensibilisées aux enjeux de l'OA que la majorité des chercheurs. Il convient donc de s'appuyer sur elles pour les accompagner dans cette mutation.

\subsubsection{Soutenir les SCD dans leur rôle d'accompagnateur au dévelop- pement de l'OA}

Dans la majorité des organisations, et les SCD ne font pas exception, l'allocation du temps du personnel est très largement fonction de l'ancienneté des activités. Le soutien au développement de l'OA faisant partie des activités les plus récentes, il est trop souvent perçu comme une activité " en plus », à laquelle on consacre le peu de temps laissé par le reste.

\section{Une équipe dédiée à l'OA}

Si les SCD veulent pouvoir jouer leur rôle, ils doivent dédier une équipe à l'OA, déchargée de toute autre fonction. La taille et l'organisation de cette équipe varieront naturellement selon celles de l'établissement que dessert le SCD. Au minimum, et en tenant compte de sa propre force de travail, cette équipe devra définir avec les laboratoires une politique de dépôt en archive ouverte adaptée à leurs souhaits et possibilités :

- dépôt par le chercheur lui-même

- dépôt par un personnel ITRF du laboratoire

- dépôt par un personnel du SCD

Quelle que soit l'organisation retenue, l'équipe OA interviendra en soutien, à la demande du chercheur ou du laboratoire. En l'absence de demande ponctuelle, un point annuel avec le laboratoire permettra de garder le contact.

Si l'établissement ou certains de ses laboratoires souhaitent créer une revue en OA, l'équipe OA du SCD devra appuyer cette initiative, voire la suggérer si les conditions semblent favorables. 


\section{Une équipe formée à l'OA}

Pour être efficace, l'équipe OA du SCD doit être formée. Le stage Enssib « Libreaccès et archive ouverte : boîte à outils » constitue une bonne base de départ. Il pourra être complété à la carte par l'offre des URFIST.

\subsubsection{S'appuyer sur les SCD pour garantir la qualité des métadonnées et des référentiels}

Si l'OA est un sujet récent pour les bibliothécaires, la qualité des métadonnées et des référentiels fait partie de leur culture professionnelle de longue date, sous les noms plus traditionnels de catalogage et de gestion des autorités. L'harmonisation des métadonnées et le contrôle de la qualité de l'archive ouverte de l'établissement ne nécessitent donc pas de compétences nouvelles, mais simplement l'adaptation à un nouvel environnement de compétences largement répandues dans les SCD.

HAL, qui est la plateforme d'archive ouverte la plus utilisée dans l'ESR français, a été conçue pour permettre au chercheur de déposer lui-même ses publications, avec une grande liberté. Mais la gestion des métadonnées et des référentiels ne fait pas partie de son coeur de métier. C'est au bibliothécaire de prendre le relais à partir ce cette étape.

\subsection{Ajouter des fonctionnalités complémentaires à HAL}

Depuis son ouverture, en 2001, HAL a fait l'objet d'améliorations régulières. Certaines fonctionnalités manquent cependant pour en tirer le meilleur parti.

\subsubsection{Introduire des savoir-faire répandus dans le monde des biblio- thèques pour la gestion des référentiels}

Bien qu'elle se soit améliorée avec la création d'AURéHAL, ${ }^{19}$ la gestion des référentiels de HAL n'offre pas encore le niveau de fonctionnalité requis. Il manque en particulier le moyen de suivre l'évolution des structures de recherche, qui, comme toutes les organisations, ont une vie. Elles peuvent fusionner, changer d'institution de rattachement, changer de nom, passer du statut d'équipe à celui de laboratoire et réciproquement. La vie des structures de recherche a de nombreuses similitudes avec celle des titres de périodiques. Les bibliothécaires, qui parlent plus spontanément d'autorités que de référentiels, ont mis au point des savoir-faire pour retracer ces vies et identifier les sources d'information utilisées pour le faire.

Un travail d'analyse concerté entre le CCSD et des bibliothécaires spécialistes de la gestion des autorités pourrait permettre d'importants progrès dans ce domaine.

\subsubsection{Produire les données de l'ESGBU avec le module statistique de HAL}

Depuis sa dernière actualisation, à partir des données de l'année 2013, l'ESGBU recueille des données sur les archives ouvertes. Le module statistique de HAL permet d'extraire de nombreuses informations de l'archive ouverte d'une établissement. Mais les données à recueillir pour l'ESGBU et les extractions possibles avec HAL ne sont

19. AURéHAL : Accès Unifié aux Référentiels HAL. URL : https://aurehal.archives-ouvertes.fr/ (visité le 12/04/2018). 
pas exprimées dans les mêmes formes. Il en résulte un fort doute sur l'homogénéité des résultats publiés par les différents établissements.

Là encore, un travail d'analyse concerté entre le CCSD, le DISTRD et la commission évaluation de l'ADBU permettrait de s'entendre précisement sur ce qu'on compte et d'aboutir à la création d'un rapport préconfiguré dans HAL, pour obtenir en quelques clics les données utiles à l'ESGBU. La refonte du module statistiques de HAL fait partie des priorités du CCSD pour 2018, qui a fait une enquête utilisateurs à ce sujet. ${ }^{20}$

\subsubsection{Faire une revue systématique de l'interface de HAL}

Enfin, il serait intéressant de faire des tests d'utilisabilité de HAL avec des publics différents : chercheurs, étudiants avancés, professionnels de l'information...

Ces tests permettraient une amélioration de l'interface et, par là même d'élargir le public susceptible d'utiliser cet outil fédérateur.

\subsection{Créer une plateforme fédératrice de l'action des biblio- thèques pour l'Open Access en France}

La rédaction du présent rapport a montré que les initiatives françaises en faveur de l'OA était nombreuses mais peu coordonnées. La création d'une plateforme fédératrice de l'action des bibliothèques pour l'OA en France permettrait à tous de bénéficier de l'effort de chacun, dans le respect des acteurs et de leur identité.

\subsubsection{Porte d'entrée}

Porte d'entrée de l'OA pour les bibliothèques de France, cette plateforme ferait principalement de la veille et de l'agrégation de ressources validées. En tant que plateforme, elle comprendrait une partie contributive ouverte à tous les acteurs, avec modération par l'institution porteuse. On pourrait proposer aux utilisateurs de signaler les contenus qui leur ont été le plus utiles, par exemple par l'attribution d'une à cinq étoiles.

Subsidiairement, la plateforme pourrait aussi produire de nouvelles ressources et améliorer des ressources existantes.

La plateforme s'appuyerait sur les principaux sites web existants :

- Couperin (outillage mutualisé)

- OA week France (annuaire des événements)

- HAL

Les bibliothécaires pourraient y trouver facilement des ressources dont ils ont besoin pour remplir leur mission de soutien aux chercheurs :

- le droit de l'OA;

- les différentes formes de dépôt en archive ouverte;

- les différentes formes de publication directe en $\mathrm{OA}$;

- les actions de communication en faveur de l'OA;

— des retours d'expériences...

20. Agnès Magron. Refonte du module statistiques : résultats de l'enquête auprès des administrateurs de portail. 16 mai 2018. URL : https://www.ccsd.cnrs.fr/2018/05/refonte-du-module-statistique s-resultats-de-lenquete-aupres-des-administrateurs-de-portail/ (visité le 17/05/2018). 


\subsubsection{Observatoire}

La plateforme comprendrait aussi un observatoire de l'OA en France. Les données collectées pour la réalisation du présent rapport en constitueraient le point de départ. Afin d'éviter qu'elles se périment rapidement, elles devront faire l'objet d'une structuration, d'une actualisation et d'un enrichissement, sous la forme d'une base de données. Cette base devra pouvoir être consultée via une interface conviviale à créer, permettant une recherche à facettes (établissements, outils, manifestations, disciplines, fourchette de dates...). Elle pourra également servir de source à la publication d'un état des lieux annuel, permettant ainsi de suivre l'évolution de l'OA en France.

\subsubsection{Intégration et portage}

Cette plateforme aurait toute sa place au sein du site Ouvrir la science, lancé par le CoSO fin 2018. ${ }^{21}$

Elle pourrait être portée par l'Université de Lyon, dans le cadre de l'IDEXLYON (4. Actions transversales Recherche / Action 3. Politique globale des publications scientifiques / Politique d'Open access intégrée), en lien avec la MSH Lyon Saint-Eienne et avec l'Enssib, qui pourrait en assurer l'hébergement et la gestion.

L'intérêt d'un portage opérationnel par l'Enssib est triple :

1. inscription dans les orientations stratégiques de l'établissement, telles que définies dans son contrat avec l'Etat pour la période 2016-2020 ${ }^{22}$;

2. position de l'Enssib comme établissement de référence pour la communauté professionnelle des bibliothèques et de la documentation, avec une forte activité de veille scientifique, une fonction d'observatoire des pratiques et des usages et un projet de développement d'une plateforme nationale et internationale de ressources librement accessibles;

3. articulation avec le volet formation (initiale et continue) des acteurs de la science ouverte en bibliothèque.

Si ce projet voit le jour, il conviendra naturellement d'en préciser les modalités de gouvernance, associant les parties prenantes de l'OA en France (ABES, ADBU, CasuHal, CCSD, Couperin, CPU, GIS Collex-Persée, GIS Urfist, INIST...), et de réalisation technique.

21. CoSO, Ouvrir la Science, op. cit.

22. Voir les extraits du Contrat de site 2016-2020 Université de Lyon et du Projet d'établissement Enssib 2020 en annexe au présent rapport. 


\section{Conclusion}

Le modèle économique actuel de la publication scientifique n'est plus soutenable depuis plusieurs années. Les établissements d'ESR ne peuvent plus continuer à payer toujours plus cher à un oligopole pour pouvoir accéder à des publications qui ne sont que la phase ultime d'un processus qu'ils ont entièrement financé.

Certaines alternatives apparentes ont montré leurs limites. La tentative d'abonnement titre à titre en lieu et place des bouquets est une oeuvre complexe, qui aboutit à un appauvrissement de l'offre à disposition des chercheurs. Les négociations avec les grands éditeurs commerciaux pour la transition du modèle par abonnement vers un modèle de Gold OA avec APC, comme au Royaume-Uni et dans d'autres pays européens, atteint rapidement ses limites. Les prix ne baissent pas, ils augmentent même trop souvent. De ce fait, les négociations s'enlisent de plus en plus.

Les solutions sont donc à chercher du côté de l'OA, tel que décrit dès 2002 par la déclaration de Budapest, qui prévoit deux voies, dites « verte » et « dorée »;

La voie verte, ou Green OA, est le dépôt par les chercheurs de leurs publications dans des dépôts d'archive ouverte. Le contexte juridique français s'est fortement éclairci dans une direction favorable au Green OA depuis la loi $\mathrm{n}^{\circ}$ 2016-1321, dite Loi Lemaire. La France a de plus la chance de disposer de HAL, une plateforme nationale maintenue, pérenne et évolutive. HAL est à la fois une archive ouverte pluridisciplinaire et un ensemble de portails d'archives institutionnelles pour les établissements d'ESR. Mais tous les chercheurs n'ont pas forcément la même aisance, la même appétence et la même disponibilité pour les opérations techniques de dépôt. Les bibliothèques d'ESR ont un rôle important à jouer en matière d'organisation, de facilitation et d'accompagnement.

Le Gold OA est la publication de revues nativement en OA. Contrairement à ce que la pratique de ces dernières années a pu laisser croire, il n'est par forcément financé par le paiement d'APC. Là encore, la France a la chance d'avoir un acteur de premier plan, OpenEdition, qui utilise le modèle économique du FreeMium. Quelques établissements ont également décidé d'héberger eux-mêmes quelques revues pilotées par leurs propres chercheurs. La communauté des mathématiciens a lancé une revue en Fair OA. Dans tous ces cas, les bibliothèques ont un rôle à jouer dans le développement et l'accompagnement de ce type d'initiatives.

Pour mener à bien ces nouvelles missions, les bibliothèques d'ESR ont besoin d'équipes dédiées et formées. Elle ont aussi besoin d'avoir accès à un outillage mutualisé permettant la capitalisation des efforts de chacun au bénéfice de tous, plutôt que leur dispersion. C'est dans cette optique que s'inscrit le projet de plateforme proposée à la fin du chapitre $4 \mathrm{du}$ présent rapport.

Une politique nationale, pour mettre en cohérence et multiplier les initiatives existantes commence à se mettre en place avec le CoSO et le Plan national pour la Science Ouverte. Quelle que soit l'importance de l'implication des bibliothèques dans le dévelop- 
pement de l'OA, la question cruciale de l'évaluation de la recherche et des carrières des chercheurs ne sera jamais de leur ressort et ne pourra être réglée que par en haut.

Le développement de l'OA est une oeuvre de longue haleine pour un enjeu de la plus haute importance. Dans le domaine de la description bibliographique, la synergie des initiatives locales et de la politique nationale a permis, sur plusieurs décennies, d'aboutir au SUDOC, dont plus personne ne saurait se passer aujourd'hui. Nous avons aujourd'hui les moyens de réussir l'OA si nous nous en emparons tous. 


\section{Résumé des propositions}

\section{Communiquer largement sur les objectifs et possibilités de diffusion des travaux de la recherche}

1. Rappeler l'article L112-1 du code de la recherche, qui traite des objectifs de la recherche publique, dont font partie le partage et la diffusion des connaissances et des données scientifique, en priorité à des formats libres d'accès.

2. Rappeler l'article L533-4 du code de la recherche, qui donne aux chercheurs le droit de déposer leurs écrits scientifiques en archive ouverte.

3. Rappeler que le programme européen de financement de la recherche H2020, qui comporte l'obligation d'assurer le libre accès aux publications issues des recherches auxquelles elle aura attribué un financement, même partiel, en Green ou en Gold OA.

4. Mener des campagnes nationales de communication régulières, notamment à l'occasion de l'Open Access Week, sur les établissements ayant adopté un mandat de libre accès et/ou créé des services autour de l'OA.

\section{Rendre la publication en OA incitative pour la carrière des chercheurs}

5. Sensibiliser chacune des sections du CNU afin qu'elles intègrent la publication en OA dans leurs critères d'évaluation.

6. Engager le dialogue entre le MESRI et le HCERES pour qu'il considère l'OA comme un critère important d'évaluation.

7. Inciter l'ANR à faire de la publication en OA une obligation pour les projets qu'elle finance, à l'instar de H2020.

8. Inciter les conférences d'établissements (CPU, CDEFI et CGE) à mettre en place un dispositif d'accompagnement de leurs établissements membres pour qu'ils formalisent et mettent en oeuvre leur propre politique d'OA.

9. Suivre le déroulement des travaux du groupe-projet « Évaluation de la Recherche » du CoSO et prendre des mesures opérationnelles à partir de ses propositions.

10. Etudier la transposition à l'évaluation des chercheurs français du rapport de la Commission européenne Evaluation of Research , Careers fully acknowledging, Open Science practices 


\section{Accélérer la prise en compte de l'OA par les établisse- ments}

11. Lancer un appel à projet MESRI pour un label « Université promotrice de l'Open Access ».

12. Rendre la définition d'une politique d'OA obligatoire pour les établissements d'enseignement supérieur et de recherche et les organismes de recherche

\section{Renforcer les services communs de documentation (SCD) dans leur rôle d'acteur du développement de l'OA}

13. Soutenir les SCD dans leur rôle d'accompagnateur au développement de l'OA, en créant dans chaque SCD une équipe dédiée et formée à l'OA.

14. S'appuyer sur les SCD pour garantir la qualité des métadonnées et des référentiels.

\section{Ajouter des fonctionnalités complémentaires à HAL}

15. Introduire des savoir-faire répandus dans le monde des bibliothèques pour la gestion des référentiels.

16. Produire les données de l'ESGBU avec le module statistique de HAL.

17. Faire une revue systématique de l'interface de HAL.

\section{Créer une plateforme fédératrice de l'action des biblio- thèques pour l'Open Access en France}

18. Porte d'entrée de l'OA pour les bibliothèques de France, cette plateforme ferait principalement de la veille et de l'agrégation de ressources validées. Subsidiairement, la plateforme pourrait aussi produire de nouvelles ressources et améliorer des ressources existantes. La plateforme comprendrait aussi un observatoire de l'OA en France, base de données servant également de source à la publication d'un état des lieux annuel. 
Annexes 



\section{Annexe A}

\section{Données : recensement des archives ouvertes des établissements d'enseignement supérieur et de recherche et des organismes de recherche}

\section{Méthode de constitution du corpus}

Le corpus a été constitué du 21 au 30 novembre 2017 puis actualisé du 29 novembre au 4 décembre 2018.

Pour chaque archive ouverte repérée, les données suivantes ont été relevées :

- url

- nombre de documents (D)

- nombre de notices $(\mathrm{N})$

- date d'ouverture

Ces données ont été stockées dans un tableau au format ODS, permettant ainsi le calcul de totaux et la réalisation des graphiques qui figurent dans le corps du présent document.

Ce recueil de données pourrait servir de point de départ à une liste officielle des archives ouvertes, avec un appel à contribuer : « vous connaissez une archive ouverte qui n'est pas dans liste : signalez-la ».

\section{Etablissements d'enseignement supérieur et de recherche}

Seule l'approche par statut permet d'avoir une liste contrôlée des établissements d'enseignement supérieur et de recherche. La source utilisée est la Liste des établissements d'enseignement supérieur et de recherche ${ }^{1}$

Les établissements y sont listés par statut administratif.

Le corpus ainsi constitué permet d'établir un tableau de 154 établissements et 6 associations, avec un code unique à trois caractères :

1. http://www .enseignementsup-recherche.gouv.fr/cid49705/liste-des-etablissements-denseignement-superieur-et-de-recherche.html (visité le 21/11/2017) 
- une lettre indiquant le statut

- deux chiffres par ordre séquentiel

\begin{tabular}{|l|l|l|}
\hline Code de l'éducation & Statut & Racine code établissement \\
\hline D 711-1 & 1 . Université & U \\
\hline D 711-6 & 2 . ComUE & C \\
\hline D 718-5 & Associations & A \\
\hline D 711-2 & 3. Instituts et écoles & I \\
\hline D 716-1 & 4 ENS & N \\
\hline D 717-1 à D 717-3 & 5 . Grands établissements & G \\
\hline D 745-5 et D 741-7 & 6. ENSI et ENI & E \\
\hline D 741-9 & 7 IEP & P \\
\hline
\end{tabular}

Cependant, plusieurs types d'établissements comparables peuvent relever de statuts différents (par exemple, les 10 IEP relèvent de 3 statuts différents), d'où la nécessité de leur attribuer un «type » correspondant mieux à leur réalité.

\begin{tabular}{|l|l|}
\hline Type & Code employé dans le tableau \\
\hline Association & Association \\
\hline ComUE & ComUE \\
\hline Ecoles & Ecole \\
\hline Ecole normale supérieure & ENR \\
\hline Etablissement de recherche & ER \\
\hline Institut d'études politiques & IEP \\
\hline Institut national des sciences appliquées & INSA \\
\hline Institut national polytechnique & INP \\
\hline Université & Université \\
\hline Université de technologie & UT \\
\hline
\end{tabular}

Il existe aussi un jeu de données intitulé Principaux établissements d'enseignement supérieur $^{2}$. Comprenant 251 enregistrements, il est cependant difficile à utiliser pour cette étude, en raison de son ordre de classement non évident. Il inclut des établissements d'enseignement supérieur privé et les ESPE, bien qu'elles ne soient pas des établissements, mais des composantes d'université.

\section{Organismes de recherche}

Il est difficile d'obtenir une liste officielle des organismes de recherche. Ce qui s'en approche les plus est la page Organismes de recherche : Liste des principaux établissements publics de recherche ${ }^{3}$. Bien que n'étant pas principalement un établissement de recherche, la Bibliothèque nationale de France (BNF) a une activité de recherche et a été comptée avec les organismes de recherche, afin d'éviter de créer une catégorie supplémentaire.

2. https://data.enseignementsup-recherche.gouv.fr/explore/dataset/fr-esr-principauxetablissements-enseignement-superieur (visité le $21 / 11 / 2017$ )

3. http://www.enseignementsup-recherche.gouv.fr/pid24575-cid49677/principauxetablissements-publics-de-recherche-et-d-enseignement-superieur.html (visité le 21/11/2017) 


\begin{tabular}{|l|l|l|}
\hline Code de l'éducation & Statut & Racine code établissement \\
\hline (sans objet) & 8 . Organisme de recherche & $\mathrm{O}$ \\
\hline
\end{tabular}

Un code a été attribué aux organismes de recherche, afin de leur permettre de figurer dans le même tableau que les établissements d'enseignement supérieur et de recherche.

\begin{tabular}{|l|l|}
\hline Type & Code employé dans le tableau \\
\hline Organisme de recherche & Organisme \\
\hline
\end{tabular}

\section{Protocole}

Une première recherche est effectuée dans :

https://hal.archives-ouvertes.fr/browse/portal

Cette liste n'a pas de classement strict apparent, mais elle est globalement dans l'ordre chronologique d'ouverture des portails. Elle ne distingue pas les portails thématiques des portails d'institutions.

Puis dans :

https://hal.archives-ouvertes.fr/browse/collection

En l'absence de portail et de collection dans HAL, une recherche est effectuée dans le moteur de recherche Qwant avec archives ouvertes + nom de l'établissement recherché.

\section{A.1 Universités}

La liste est classée par nom officiel, dans l'ordre de l'article D711-1 du Code de l'éducation. Le nom officiel est suivi du nom d'usage lorsqu'il est différent.

\section{A.1.1 Universités à statut ordinaire}

\section{Aix-Marseille = Aix-Marseille Université}

Portail HAL ouvert le 17 décembre 2013

https://hal-amu.archives-ouvertes.fr

D 26324 / N 68669

\section{Amiens = Université de Picardie Jules-Verne \\ (pas d'AO trouvée)}

\section{Angers}

AOI : Open Knowledge, INformation, Access (OKINA)

http://okina.univ-angers.fr/

Ouvert en avril $2014^{4}$

Pas de statistiques affichées. On peut cependant utiliser la recherche avancée :

— toutes les notices : 16368 références

— uniquement les notices avec fichier : 2786 références

4. http://www.univ-angers.fr/fr/recherche/actualites/open-access.html （visité le $24 / 11 / 2017)$ 
Export possible aux formats BibTex, Endnote et RIS

Antilles et Guyane $=$ Université des Antilles

Malgré son nom officiel, l'université de Guyane n'en fait plus partie depuis 2015.

Portail HAL ouvert le 15 mai 2011

https://hal.univ-antilles.fr

D 2817 / N 5476

\section{Artois}

Portail HAL ouvert le 6 avril 2009

https://hal-univ-artois .archives-ouvertes.fr

D 1316 / N 3240

Avignon $=$ Université d'Avignon et des Pays de Vaucluse

Portail HAL ouvert le 2 février 2010

https://hal-univ-avignon.archives-ouvertes.fr

D 3188 / N 9585

\section{Besançon = Université de Franche-Comté}

Portail HAL ouvert le 6 février 2015

https://hal-univ-fcomte.archives-ouvertes.fr

D 4924 / N 19229

\section{Bordeaux}

Collections dans HAL

https://hal .archives-ouvertes .fr/UNIV-BORDEAUX

D 3973 / N 6602

AOI en projet $^{5}$

Bordeaux-III = Université Bordeaux-Montaigne

(pas d'AO trouvée)

\section{Brest $=$ Université de Bretagne-Occidentale}

Portail HAL ouvert le 11 avril 2008

http://hal.univ-brest.fr

D 7025 / N 25819

\section{Bretagne-Sud}

Portail HAL ouvert le 6 février 2015

https://hal-univ-ubs .archives-ouvertes.fr

D 969 / N 3404

5. http://bibliotheques.u-bordeaux.fr/Vos-services/Valorisation-des-publicationsscientifiques/Projet-Archive-Ouverte-Institutionnelle (visité le 30/11/2017) 


\section{Caen $=$ Université Caen-Normandie}

Portail HAL ComUE Normandie université

\section{Cergy-Pontoise}

(pas d'AO trouvée)

\section{Chambéry $=$ Université Savoie Mont Blanc}

Portail HAL ouvert le 17 juin 2009

http://hal.univ-smb.fr

D 6040 / N 20094

+ Portail HAL ComUE Université Grenoble-Alpes

\section{Clermont-Auvergne}

Portail HAL ouvert le 16 octobre 2013

https://hal-clermont-univ.archives-ouvertes.fr

D 8583 / N 29064

\section{Corse $=$ Université de Corse-Pascal-Paoli}

Portail HAL ouvert le 1er février 2012

https://hal-univ-corse.archives-ouvertes.fr

D 441 / N 3097

Dijon $=$ Université de Bourgogne

Portail HAL ouvert le 2 avril 2010

https://hal-univ-bourgogne.archives-ouvertes.fr

D 5984 / N 25517

\section{Évry-Val-d'Essonne}

Portail HAL ouvert le 6 octobre 2016

https://hal-univ-evry.archives-ouvertes.fr

D 1724 / N 4018

\section{Grenoble-Alpes}

Portail HAL ComUE Université Grenoble-Alpes

\section{La Guyane}

Portail HAL ouvert le 12 octobre 2016

https://hal-univ-guyane.archives-ouvertes.fr

D 353 / N 553 


\section{La Réunion}

Portail HAL ouvert le 4 mars 2015

http://hal . univ-reunion.fr

D 2326 / N 6878

\section{La Rochelle}

Portail HAL ouvert le 1er juin 2017

https://hal-univ-rochelle.archives-ouvertes.fr

D 2291 / N 4211

\section{Le Havre}

Portail HAL ComUE Normandie université

\section{Le Mans = Université du Maine}

Portail HAL ouvert le 23 février 2017

https://hal-univ-lemans .archives-ouvertes.fr

D 3397 / N 8307

\section{Lille}

L'Université de Lille résulte de la fusion de Lille I, II et III à compter du 1er janvier $2018^{6}$.

L'Université Lille-III Charles-de-Gaulle a un portail HAL ouvert le 1er février 2013 http://hal univ-lille3.fr

D 3453 / N 7432

La nouvelle université fusionnée a ouvert une AOI, LillOA en 2018 https://lilloa.univ-lille.fr/

Pas de statistiques affichées. La recherche donne 2665 réponses, incluant les documents et les notices.

LillOA est connecté à un portail HAL

https://hal.univ-lille.fr/

D 7889 / N 32592

\section{Limoges}

Portail HAL ouvert le 21 mai 2008

https://hal-unilim.archives-ouvertes.fr

D 2390 / N 17770

\section{Littoral = Université du Littoral-Côte-d'Opale}

Collection dans HAL

urlhttps ://hal.archives-ouvertes.fr/UNIV-LITTORAL

D 1117 / N 3479

6. Décret 2017-1329 du 11 septembre 2017 


\section{Lyon-I = Université Claude-Bernard-Lyon-I}

Portail HAL ouvert le 1er février 2018

https://hal-univ-lyon1.archives-ouvertes.fr

D 18485 / N 58549

+ Portail HAL ComUE Université de Lyon

\section{Lyon-II = Université Lumière-Lyon-II}

Collection dans HAL

https://hal.archives-ouvertes.fr/UNIV-LYON2

D 10921 / N 40977

+ Portail HAL ComUE Université de Lyon

\section{Lyon-III = Université Jean-Moulin-Lyon-III}

Portail HAL ouvert le 12 septembre 2008

https://hal-univ-lyon3.archives-ouvertes.fr

D 3801 / N 22224

+ Portail HAL ComUE Université de Lyon

\section{Marne-la-Vallée = Université Paris-Est Marne-la-Vallée}

Portail HAL commun avec Paris XII (Paris-Est Créteil Val-de-Marne) ouvert le 15 février 2011

https://hal-upec-upem.archives-ouvertes.fr

D 10875 / N 26250

\section{Montpellier}

Portail HAL ouvert le 22 mai 2017

https://hal-umontpellier.archives-ouvertes.fr

D 12985 / N 38766

\section{Montpellier-III = Université Paul-Valéry-Montpellier-III}

AOI à accès via le site web de la BU. Migration vers HAL prévue ${ }^{7}$.

\section{Mulhouse = Université de Haute-Alsace}

Collection dans HAL

https://hal archives-ouvertes.fr/SCD-UHA

D 1892 / N 803

+ AOI association Alsace

7. https://www.open-access.fr/actualites/12-1-archive-ouverte-de-l-upvm （visité le $29 / 11 / 2018)$ 


\section{Nantes}

Portail HAL ouvert le 8 juin 2009

http://hal.univ-nantes.fr

D 13206 / N 32196

\section{Nice $=$ Université Nice Sophia Antipolis}

Le portail de l'Université de Nice Sophia Antipolis, ouvert le 8 octobre 2007, est devenu en 2018 le portail de la ComUE Université Côte d'Azur.

\section{Nîmes}

Collection dans HAL

https://hal .archives-ouvertes.fr/UNIMES/

D 181 / N 367

\section{Nouvelle-Calédonie}

\section{AOI}

http://orioai.univ-nc.nc/search/index.html

Pas de statistiques

\section{Orléans}

Portail HAL ouvert le 2 octobre 2015

https://hal-univ-orleans .archives-ouvertes.fr

D 6816 / N 12840

\section{Paris-I = Université Panthéon-Sorbonne}

Portail HAL ouvert le 1er novembre 2007

https://hal-paris1.archives-ouvertes.fr

D 14408 / N 24711

Paris-II = Université Panthéon-Assas

(pas d'AO trouvée)

\section{Paris-III = Université Sorbonne-Nouvelle}

Portail HAL ouvert le 14 septembre 2015

https://hal-univ-paris3.archives-ouvertes.fr

D 5634 / N 16559

\section{Sorbonne Université}

Sorbonne Université résulte de la fusion de Paris-IV (= Université Paris-Sorbonne) et Paris-VI (= Université Pierre-et-Marie-Curie) à compter du 1er janvier $2018^{8}$.

8. Décret 2017-596 du 21 avril 2017 
Paris-VI (= Université Pierre-et-Marie-Curie) a un portail HAL ouvert le 17 septembre 2010, devenu le portail de Sorbonne Université après la fusion.

https://hal. sorbonne-universite.fr/

D 38431 / N 74746

\section{Paris-V = Université Paris-Descartes}

Portail HAL ouvert le 25 octobre 2007

https://hal-descartes.archives-ouvertes.fr

D 6570 / N 8584

\section{Paris-VII = Université Paris-Diderot}

Portail HAL ouvert le 7 octobre 2011

https://hal-univ-diderot.archives-ouvertes.fr

D 22917 / N 45104

\section{Paris-VIII = Université Vincennes-Saint-Denis}

Portail HAL ouvert le 5 juillet 2012

https://hal-univ-paris8.archives-ouvertes.fr

D 4071 / N 17572

+ Portail ComUE Université Paris Lumières

\section{Paris-X = Université Paris-Ouest-Nanterre-La Défense}

Portail HAL ouvert le 5 juillet 2016

https://hal-univ-paris10.archives-ouvertes.fr

D 7246 / N 29634

+ Portail ComUE Université Paris Lumières

Paris-XI = Université Paris-Sud

Collection ComUE Paris-Saclay

\section{Paris-XII = Université Paris-Est Créteil Val-de-Marne}

Portail HAL commun avec Paris-Est Marne-la-Vallée (UPEM) ouvert le 15 février 2011

https://hal-upec-upem.archives-ouvertes.fr

D 10875 / N 26250

\section{Paris-XIII = Université Paris-XIII-Nord}

Portail HAL ouvert le 8 décembre 2009

https://hal-univ-paris13.archives-ouvertes.fr

D 4310 / N 5485 
Pau $=$ Université de Pau et des Pays de l'Adour

Portail HAL ouvert le 6 février 2018

https://hal-univ-pau.archives-ouvertes.fr/

D 2784 / N 11456

\section{Perpignan}

Portail HAL ouvert le 19 mai 2015

https://hal-univ-perp.archives-ouvertes.fr

D 1681 / N 3502

\section{Poitiers}

Portail HAL ouvert le 14 septembre 2017

https://hal-univ-poitiers archives-ouvertes.fr

D 3663 / N 13191

\section{Polynésie française}

Portail HAL ouvert le 18 octobre 2017

https://hal-upf.archives-ouvertes.fr/

D 305 / N 522

Reims = Université de Reims-Champagne-Ardenne

Portail HAL ouvert le 6 octobre 2017

https://hal-urca.archives-ouvertes.fr/

D 1370 / N 3349

\section{Rennes-I}

Portail HAL ouvert le 1 décembre 2012

https://hal-univ-rennes 1 archives-ouvertes.fr

D 20387 / N 54255

Rennes-II = Université Rennes-II-Haute-Bretagne

Portail HAL ouvert le 23 février 2017

https://hal-univ-rennes 2 archives-ouvertes.fr

D 6050 / N 18625

\section{Rouen}

Portail HAL ComUE Normandie université

Saint-Étienne = Université Jean-Monnet-Saint-Étienne

Portail HAL ouvert le 26 janvier 2006

https://hal-ujm.archives-ouvertes.fr

D 7729 / N 29523

+ Portail HAL ComUE Université de Lyon 


\section{Strasbourg}

AOI association Alsace

\section{Toulon}

Portail HAL ouvert le 22 juillet 2013

https://hal-univ-tln.archives-ouvertes.fr

D 4082 / N 11625

\section{Toulouse-I = Université Toulouse-I-Capitole}

\section{AOI}

http://publications.ut-capitole.fr

D 4039 / N 19453

Toulouse-II = Université Toulouse-Jean-Jaurès

Portail HAL ouvert le 15 mai 2011

https://hal-univ-tlse2.archives-ouvertes.fr

D 10667 / N 12725

Université de Toulouse-III = Université Toulouse-III-Paul-Sabatier

Portail HAL ouvert le 15 mai 2011

https://hal-univ-tlse3.archives-ouvertes.fr

D 18705 / N 24681

\section{Tours = Université François-Rabelais}

Portail HAL ouvert le 24 juillet 2013

https://hal-univ-tours .archives-ouvertes.fr

D 3975 / N 15546

Valenciennes = Université de Valenciennes et du Hainaut-Cambrésis = Université polytechnique Hauts-de-France

Portail HAL [date d'ouverture non mentionnée, probablement en 2018]

https://hal-uphf .archives-ouvertes.fr

D 1305 / N 10021

AOI Theoreme (Thèses, Open access, Recherche, Mémoires)

https://theoreme.uphf.fr

D 435

\section{Versailles-Saint-Quentin-en-Yvelines}

Portail HAL ouvert le 15 juillet 2015

https://hal-uvsq.archives-ouvertes.fr

D 6015 / N 11659 


\section{Institut national polytechnique de Toulouse}

\section{OAI}

http://oatao.univ-toulouse.fr

Affiche ses statistiques : 15127 « eprints » presque tous en texte intégral

\section{A.1.2 Universités à statut dérogatoire}

Bien qu'assimilables à plusieurs égards à des universités, les établissements suivants ont le statut de grand établissement :

- Université Paris-Dauphine

- Université de Lorraine

- Institut polytechnique de Grenoble

— Institut polytechnique de Bordeaux

\section{A.2 ComUEs et associations}

La composition des ComUEs et associations est disponible sur le site du ministère ${ }^{9}$.

\section{A.2.1 ComUEs}

Sur 19 ComUEs, 6 ont un portail dans HAL, 4 ont une collection dans HAL et aucune n'a d'archive ouverte institutionnelle.

Lors que le portail HAL est porté par la ComUE, certains établissements membres ont parfois leur portail propre, généralement créé avant celui de la ComUE.

\section{ComUEs avec un portail dans HAL}

\section{Communauté Université Grenoble Alpes}

http://hal.univ-grenoble-alpes.fr

Portail HAL ouvert le 10 juillet 2013

D 41106 / N 95541

L'Université de Chambéry, associée, a son propre portail HAL, ouvert en 2009.

\section{Normandie Université}

Portail HAL ouvert le 19 mai 2015

https://hal-normandie-univ.archives-ouvertes.fr

D 12555 / N 32343

\section{Université Côte d'Azur}

Le portail de l'Université de Nice Sophia Antipolis, ouvert le 8 octobre 2007, est devenu en 2018 le portail de la ComUE Université Côte d'Azur.

https://hal.univ-cotedazur.fr

D 13989 / N 23504

9. http://www.enseignementsup-recherche.gouv.fr/cid94756/les-regroupementsuniversitaires-et-scientifiques-une-coordination-territoriale-pour-un-projet-partage. html $\% 20-\% 20$ Liste_des_etablissements_membres_des_COMUE_et_des_associations (visité le $04 / 12 / 2018)$ 


\section{Université de Lyon}

Portail HAL ouvert le 14 mars 2017

https://hal-udl.archives-ouvertes.fr

D 23588 / N 83716

Plusieurs membres ont leur propre portail dans HAL :

1. Université Claude Bernard Lyon 1, ouvert en 2018

2. Université Jean Moulin Lyon 3, ouvert en 2008

3. Université Jean Monnet - Saint-Étienne, ouvert en 2006

4. ENS de Lyon, ouvert en 2006

\section{Université Paris Lumières}

Portail HAL ouvert le 1er juin 2017

https://hal-univ-paris-lumieres . archives-ouvertes.fr

D 10035 / N 38728

Plusieurs membres ont leur propre portail dans HAL :

1. Université Paris 8 Vincennes Saint-Denis, ouvert en 2012

2. Université Paris Ouest Nanterre-La Défense, ouvert en 2016

\section{Université Paris Seine}

Portail HAL ouvert le 14 mai 2018

https://hal-u-paris-seine.archives-ouvertes.fr/

D 3806 / N 7088

\section{ComUE avec une collection dans HAL}

\section{HESAM Université}

Collection dans HAL

https://hal .archives-ouvertes.fr/UNIV-HESAM

D 38704 / N 68526

Université de recherche Paris sciences et lettres - PSL Research University

Collection dans HAL

https://hal .archives-ouvertes.fr/PSL

D 45648 / N 89496

\section{Université Paris-Saclay}

Collection dans HAL

https://hal . archives-ouvertes .fr/UNIV-PARIS-SACLAY

D 17375 / N 31489

\section{Université Sorbonne Paris Cité}

Collection dans HAL

https://hal.archives-ouvertes.fr/USPC

D 43604 / N 76218 


\title{
ComUEs sans archive ouverte
}

1. Communauté d'universités et établissements d'Aquitaine

2. Communauté d'universités et établissements Centre-Val de Loire

3. Communauté d'universités et établissements Lille Nord de France

4. Languedoc-Roussillon Universités

5. Université de Bourgogne Franche-Comté

6. Université Bretagne Loire

7. Université confédérale Léonard de Vinci

8. Université fédérale de Toulouse Midi-Pyrénées

9. Université Paris-Est

\section{A.2.2 Associations}

Le nom de l'établissement pivot figure entre parenthèses après le nom de l'association. Seule l'association d'Alsace a une archive ouverte institutionnelle.

\section{Association avec archive ouverte institutionnelle}

\section{Alsace (université de Strasbourg-UNISTRA)}

AOI lancée le 14 octobre 2016

https://univoak.eu/ (visité le 30 novembre 2017)

Pas de statistiques affichées, mais nombre de réponses si on lance une recherche, on trouve D 2890 / N 28920

Choix d'une AOI comme brique du futur SI recherche. Développé avec Solar, Fedora et Drupal ${ }^{10}$.

\section{Associations sans archive ouverte}

1. Aix-Marseille (Aix-Marseille université-AMU)

2. Auvergne (Université Clermont Auvergne)

3. Champagne (Université de Reims-Champagne-Ardenne)

4. Lorraine (université de Lorraine)

5. Picardie (université de Picardie Jules Verne-UPJV)

6. Sorbonne Université (Sorbonne Université)

\section{A.3 Instituts et écoles extérieurs aux universités}

\section{A.3.1 Ecoles}

\author{
Ecole centrale de Lille \\ Collection dans HAL \\ https://hal .archives-ouvertes.fr/EC-LILLE \\ D 3789 / N 15296
}

10. Source : Adeline Rege, stage Enssib le 12/12/2017 
Ecole centrale de Lyon

Collection dans HAL

https://hal .archives-ouvertes.fr/EC-LYON

D 6129 / N 10741

+ portail HAL ComUE Université de Lyon

Ecole centrale de Marseille

Collection dans HAL

https://hal .archives-ouvertes .fr/EC-MARSEILLE

D 4380 / N 12058

Ecole centrale de Nantes

Collection dans HAL

https://hal .archives-ouvertes.fr/EC-NANTES

D 5215 / N 7087

Ecole d'ingénieurs SIGMA Clermont

Collection dans HAL

https://hal archives-ouvertes.fr/SIGMA-CLERMONT

D 1628 / N 3980

Ecole nationale des travaux publics de l'Etat

Collection dans HAL

https://hal .archives-ouvertes.fr/ENTPE

D 3052 / N 6059

+ Portail HAL ComUE Université de Lyon

Ecole nationale d'ingénieurs de Saint-Etienne

Portail HAL ComUE Université de Lyon

Ecole nationale supérieure de chimie de Paris

Portail HAL ouvert le 15 mai 2011

https://hal-enscp.archives-ouvertes.fr

D 633 / N 1759

Ecole nationale supérieure de mécanique et d'aérotechnique de Poitiers (pas d'AO trouvée)

Ecole nationale supérieure d'ingénieurs de Caen

Portail HAL ComUE Normandie université 
Ecole nationale supérieure des arts et industries textiles (pas d'AO trouvée)

\section{A.3.2 Instituts}

Institut national des sciences appliquées de Lyon

Collection dans HAL

https://www . archives-ouvertes.fr/INSA-LYON

D 10525 / N 29593

+ Portail HAL ComUE Université de Lyon

Institut national des sciences appliquées de Rennes

Portail HAL ouvert le 14 mai 2014

https://hal-insa-rennes . archives-ouvertes.fr

D 4189 / N 10628

Institut national des sciences appliquées de Toulouse

Portail HAL ouvert le 14 septembre 2017

https://hal-insa-toulouse.archives-ouvertes.fr

D 7731 / N 7156

Institut national des sciences appliquées de Rouen

Portail HAL ComUE Normandie Université

Institut national des sciences appliquées de Strasbourg

Collection dans HAL

https://hal . archives-ouvertes.fr/INSA-STRASBOURG

D 1039 / N 1441

+ AOI association Alsace

Institut national des sciences appliquées Centre Val de Loire (pas d'AO trouvée)

Institut national universitaire Jean-François Champollion (pas d'AO trouvée)

Institut supérieur de mécanique de Paris (pas d'AO trouvée)

\section{A.3.3 Universités de technologie}

Université de technologie de Compiègne

(pas d'AO trouvée) 
Université de technologie de Belfort-Montbéliard

Collection dans HAL

https://hal . archives-ouvertes .fr/UNIV-BM

D 2868 / N 8329

Université de technologie de Troyes

(pas d'AO trouvée)

\section{A.4 Ecoles normales supérieures}

Ecole normale supérieure

Portail HAL ouvert le 20 octobre 2009

https://hal-ens.archives-ouvertes.fr

D 10327 / N 20737

Ecole normale supérieure de Cachan = Ecole normale supérieure Paris-Saclay

Collection HAL

https://hal .archives-ouvertes.fr/ENS-CACHAN/

D 5465 / N 10273

Ecole normale supérieure de Lyon

Portail HAL ouvert le 27 mars 2006

https://hal-ens-lyon.archives-ouvertes.fr

D 11415 / N 40470

+ portail HAL ComUE Université de Lyon

Ecole normale supérieure de Rennes

Collection HAL

https://hal.archives-ouvertes.fr/ENS-RENNES

D 271 / N 149

\section{A.5 Grands établissements}

\section{A.5.1 Article D717-1 : tutelle ESR}

Grands établissements placés sous la tutelle du ministre chargé de l'enseignement supérieur.

Collège de France

(pas d'AO trouvée) 


\section{Observatoire de Paris}

Portail HAL ouvert le 14 février 2007

https://hal-obspm.ccsd.cnrs.fr

D 2147 / N 6108

Conservatoire national des arts et métiers

(pas d'AO trouvée)

Institut national des langues et civilisations orientales

Portail HAL ouvert le 16 décembre 2015

https://hal-inalco.archives-ouvertes.fr

D 3606 / N 4129

Ecole nationale des chartes

(pas d'AO trouvée)

Ecole pratique des hautes études

Portail HAL ouvert le 15 février 2011

https://hal-ephe.archives-ouvertes.fr

D 7526 / N 23576

Institut d'études politiques de Paris

AOI : SPIRE

https://spire.sciencespo.fr/web/

23895 publications (= Notices) / Pas de statistiques sur D

Développé avec DSpace. Choix d'une plateforme unique permettant de lier les publications et les données produites par les chercheurs ${ }^{11}$.

Il existe aussi un portail HAL, non mentionné dans la liste des portails HAL. Il renvoie vers SPIRE dès la page d'accueil.

https://hal-sciencespo.archives-ouvertes.fr/

D 3613 / N 2229

Ecole nationale supérieure d'arts et métiers

AOI : SAM

http://sam.ensam.eu/

D $3021 / \mathrm{N}$ ?

date d'ouverture?

Institut de physique du Globe de Paris

Collection dans HAL

https://hal .archives-ouvertes.fr/IPGP

D 1282 / N 1844

11. Source : Cynthia Pedroja, stage Enssib le 12/12/2017 
Ecole des hautes études en sciences sociales

Collection dans HAL

https://hal.archives-ouvertes.fr/EHESS/

D 10493 / N 20093

Ecole nationale supérieure des sciences de l'information et des bibliothèques

Collection dans HAL

https://hal.archives-ouvertes.fr/ENSSIB

D 210 / N 329

\section{Université Paris-Dauphine}

Portail HAL ouvert le 16 juillet 2015

https://hal-univ-paris-dauphine.archives-ouvertes.fr

D 548 / N 2644

AOI Base Institutionnelle de Recherche de l'université Paris-Dauphine (BIRD)

https://basepub.dauphine.fr/

D 17481

\section{Institut polytechnique de Grenoble}

Portail HAL ComUE Université Grenoble Alpes

Institut polytechnique de Bordeaux

(pas d'AO trouvée)

\section{Université de Lorraine}

Portail HAL ouvert le 21 septembre 2015

https://hal.univ-lorraine.fr

D 27196 / N 44116

\section{A.5.2 Article D717-2 : tutelle mixte}

Grands établissements placés sous la tutelle conjointe des ministres chargés de l'enseignement supérieur et d'un ou plusieurs autres ministres.

Muséum national d'histoire naturelle

Portail HAL ouvert le 7 novembre 2007

https://hal-mnhn.archives-ouvertes.fr

D 3000 / N 6038

Institut national d'histoire de l'art

Collections dans HAL

https://hal .archives-ouvertes.fr/INHA/

D $30 /$ N 8 
Ecole des hautes études en santé publique

Portail HAL ouvert le 11 avril 2017

https://hal-ehesp.archives-ouvertes.fr

D 701 / N 2733

Institut national supérieur des sciences agronomiques, de l'alimentation et de l'environnement

(pas d'AO trouvée)

\section{CentraleSupélec}

Portail HAL ouvert le 4 mars 2015 (avant la fusion, Centrale Paris avait un portail HAL dès 2010)

https://hal-centralesupelec.archives-ouvertes.fr/

D 9325 / N 26209

\section{A.5.3 Article D717-3 : autres tutelles}

Grands établissements sous tutelle d'autres ministres.

Ministre chargé de l'agriculture

Institut des sciences et industries du vivant et de l'environnement (Agro Paris Tech)

Portail HAL ouvert le 2 juillet 2010

https://hal-agroparistech.archives-ouvertes.fr

D 5114 / N 21916

Institut national d'études supérieures agronomiques de Montpellier (Montpellier Sup Agro)

Portail HAL ouvert le 25 novembre 2016

https://hal-montpellier-supagro.archives-ouvertes.fr

D 2794 / N 7901

Institut national supérieur des sciences agronomiques, agroalimentaires, horticoles et du paysage (Agrocampus Ouest)

Portail HAL ouvert le 2 avril 2012

https://hal-agrocampus-ouest.archives-ouvertes.fr

D 875 / N 4324

Institut d'enseignement supérieur et de recherche en alimentation, santé animale, sciences agronomiques et de l'environnement (Vet Agro Sup)

Portail HAL ComUE Université de Lyon 
Ecole nationale vétérinaire, agroalimentaire et de l'alimentation, NantesAtlantique (ONIRIS)

(pas d'AO trouvée)

Ministre chargé de l'équipement

Ecole nationale des ponts et chaussées

Portail HAL ouvert le 15 septembre 2010

https://hal-enpc.archives-ouvertes.fr

D 9036 / N 15656

Ministre chargé de la défense

Ecole polytechnique

Portail HAL ouvert le 29 mai 2009

https://hal-polytechnique.archives-ouvertes.fr

D 11209 / N 16698

Institut supérieur de l'aéronautique et de l'espace

(pas d'AO trouvée)

Ecole navale

(pas d'AO trouvée)

Ministre chargé de la mer

Ecole nationale supérieure maritime

(pas d'AO trouvée)

Ministre chargé des sports

Institut national des sports, de l'expertise et de la performance

Portail HAL ouvert le 5 juillet 2016

https://hal-insep.archives-ouvertes.fr

D 405 / N 121

Ministre chargé de l'économie

Groupe des écoles nationales d'économie et statistique

Portail HAL ouvert le 1er mars 2012

https://hal-genes.archives-ouvertes.fr

D 681 / N 674 
Ministres chargés de l'industrie et des communications électroniques Institut Mines-Télécom

Portail HAL ouvert le 12 juin 2009

https://hal-imt.archives-ouvertes.fr

D 22623 / N 37940

\section{A.6 Ecoles nationales (supérieures) d'ingénieurs}

Ecole nationale supérieure de chimie de Lille

Collection dans HAL

https://hal archives-ouvertes .fr/ENSC-LILLE

D 201 / N 2076

Ecole nationale supérieure de chimie de Rennes

Collection dans HAL

https://hal .archives-ouvertes.fr/ENSC-RENNES

D 1693 / N 5573

Ecole nationale supérieure d'informatique pour l'industrie et l'entreprise (pas d'AO trouvée)

Ecole nationale d'ingénieurs de Brest

Collection dans HAL

https://hal.archives-ouvertes.fr/ENIB

D 2282 / N 5112

Ecole nationale d'ingénieurs de Tarbes

(pas d'AO trouvée)

\section{A.7 Instituts d'études politiques}

\section{A.7.1 IEP à statut ordinaire}

Institut d'études politiques d'Aix-en-Provence

(pas d'AO trouvée)

Institut d'études politiques de Bordeaux (pas d'AO trouvée)

Institut d'études politiques de Grenoble

Portail ComUE Université Grenoble Alpes 
Institut d'études politiques de Lille

(pas d'AO trouvée)

Institut d'études politiques de Lyon

Portail ComUE Université de Lyon

Institut d'études politiques de Rennes

Collection dans HAL

https://hal.archives-ouvertes.fr/SCIENCESPO_RENNES

D 7 / N 285

Institut d'études politiques de Toulouse

(pas d'AO trouvée)

\section{A.7.2 IEP à statut particulier}

IEP de Paris : grand établissement

IEP de Strasbourg : institut interne de l'Université de Strasbourg

IEP de Saint-Germain-en-Laye : institut interne des universités de Cergy-Pontoise et Versailles Saint-Quentin-en-Yvelines

\section{A.8 Organismes de recherche}

ADEME = Agence de l'environnement et de la maîtrise de l'énergie

Collection dans HAL

https://hal .archives-ouvertes.fr/ADEME

D $151 /$ N 297

ANDRA $=$ Agence nationale de gestion des déchets radioactifs

Collection dans HAL

https : //hal . archives-ouvertes.fr/ANDRA

D $200 /$ N 474

BRGM = Bureau de recherches géologiques et minières

Portail HAL ouvert le 19 mai 2010

https://hal-brgm.archives-ouvertes.fr

D $2900 /$ N 3146

CEA = Commissariat à l'énergie atomique et aux énergies alternatives

Portail HAL ouvert le 19 avril 2007

https://hal-cea.archives-ouvertes.fr

D 15091 / N 37662 


\section{CEE $=$ Centre d'études de l'emploi}

Le Centre d'études de l'emploi (CEE) devient, à compter du 1er octobre 2016, le Centre d'études de l'emploi et du travail (CEET) au sein du CNAM. Le CEET est un programme transversal du CNAM visant à développer la recherche pluridisciplinaire sur le travail et l'emploi, dans une perspective académique et de réponse à la demande sociale.

Collection dans HAL

https://hal.archives-ouvertes.fr/CEET

D 225 / N 342

\section{CEPH = Centre d'étude du polymorphisme humain}

Collection dans HAL

https://hal.archives-ouvertes.fr/CEPH

D $26 / \mathrm{N} 25$

CIRAD = Centre de coopération internationale en recherche agronomique pour le développement

AOI : Agritrop, ouverte en juin $2015^{12}$

https://agritrop.cirad.fr/

D 33400 / N 100000

Il existe aussi un portail HAL ouvert le 1er février 2007

http://hal.cirad.fr

D 5300 / N 10779

Cité nationale de l'histoire de l'immigration

(pas d'AO trouvée)

CNES = Centre national d'études spatiales

Collection dans HAL

https://hal .archives-ouvertes.fr/CNES

D $2671 /$ N 5863

CNRS $=$ Centre national de la recherche scientifique

Archive ouverte HAL

https://hal.archives-ouvertes.fr

Date d'ouverture : 1er mars 2002

D 549625 / N 1649933

GENOPOLE premier bioparc français dédié à la recherche en génomique, génétique et aux biotechnologies

(pas d'AO trouvée)

12. https://agritrop.cirad.fr/mention_legale.html (visité le 04/12/2018) 
IFÉ = Institut français de l'éducation

(pas d'AO trouvée)

IFREMER = Institut français de recherche pour l'exploitation de la mer

AOI : Archimer, ouverte en août $2005^{13}$

http://archimer.ifremer.fr/

D 18798 en libre accès +20059 accessibles à la demande

Il existe aussi une collection dans HAL

https://hal.archives-ouvertes.fr/IFREMER

D 2224 / N 5565

IFPEN = IFP Energies nouvelles

Portail HAL ouvert le 1er janvier 2012

https://hal-ifp.archives-ouvertes.fr

D 1018 / N 1312

IFSTTAR = Institut français des sciences et technologies des transports, de l'aménagement et des réseaux

AOI : Madis

http://madis-externe.ifsttar.fr

47283 notices dont 10263 avec texte intégral au 4 octobre 2018

Il existe aussi une collection dans HAL

https://hal .archives-ouvertes.fr/IFSTTAR

D 7264 / N 12845

INCA $=$ Institut national du Cancer

(pas d'AO trouvée)

INED = Institut national d'études démographiques

Collection dans HAL

https://hal .archives-ouvertes.fr/INED

D 289 / N 549

INERIS Institut national de l'environnement industriel et des risques

Portail HAL ouvert le 3 avril 2007

https://hal-ineris.archives-ouvertes.fr

D 2035 / N 4451

13. http://archimer.ifremer.fr/html/acces-libre2010.htm (visité le 04/12/2018) 
INRA Institut national de la recherche agronomique

AOI : ProdInra

https://prodinra.inra.fr

ProdInra contient 265156 références dont $18 \%$ avec texte intégral

Il existe aussi une collection dans HAL

https://hal.archives-ouvertes.fr/INRA

D 16290 / N 66904

INRIA Institut national de recherche en informatique et en automatique

Portail HAL ouvert le 1er avril 2004

https://hal.inria.fr

D 598632 / N 1051949

INSERM Institut national de la santé et de la recherche médicale

Portail HAL ouvert le 25 janvier 2006

http://www.hal.inserm.fr

D 21442 / N 5054

\section{Institut Curie}

Collection dans HAL

https://hal .archives-ouvertes.fr/CURIE

D 907 / N 1217

\section{Institut Pasteur}

Portail HAL ouvert le 6 février 2007

https://hal-pasteur .archives-ouvertes.fr

D 3157 / N 2712

IPEV = Institut Polaire français Paul Emile Victor

(pas d'AO trouvée)

IRD = Institut de recherche pour le développement

ex-ORSTOM

Portail HAL ouvert le 1er janvier 2007

http://hal.ird.fr

D 12237 / N 361

IRSN = Institut de radioprotection et de sûreté nucléaire

Portail HAL ouvert le 1er septembre 2007

https://hal-irsn.archives-ouvertes.fr

D $522 /$ N 877 
IRSTEA = Institut national de recherche en sciences et technologies pour l'environnement et l'agriculture

Collection dans HAL

https://hal.archives-ouvertes.fr/IRSTEA

D 4823 / N 5289

Musée du quai Branly

(pas d'AO trouvée)

ONERA = Office National d'Études et de Recherches Aéronautiques

Portail HAL ouvert le 17 décembre 2013

https://hal-onera.archives-ouvertes.fr

D 2592 / N 2280

\section{Organismes non pris en compte}

Il s'agit d'organismes cités dans la source, mais qui ne sont pas pris en compte dans le recensement des $\mathrm{AO}$, soit par ce qu'il ne s'agit à proprement parler d'organismes de recherche, soit parce qu'ils ont déjà été cités dans une autre annexe.

\section{ADIT $=$ Agence pour la diffusion de l'information technologique}

Il s'agit plus d'un service de consultants que d'un organisme de recherche.

Cité des sciences et Palais de la découverte : Universcience

Il s'agit plus d'un organisme de diffusion de la recherche que de recherche.

MNHN = Museum national d'histoire naturelle

Déjà pris en compte dans l'annexe "Grands établissements".

\section{OSEO}

Anciennement ANVAR, remplacé par BpiFrance. C'est un service à la recherche.

OST $=$ Observatoire des sciences et techniques

Intégré au HCERES depuis 2014.

RENATER = Réseau national des télécommunications pour la Technologie, l'Enseignement et la Recherche

Il s'agit plus d'un organisme de services à la recherche que de recherche. 


\section{Organismes ne figurant pas dans la source principale}

Certains organismes listés dans l'Annexe au projet de loi de finances pour 2018 : rapport sur les politiques nationales de recherche et de formations supérieures ${ }^{14}$ ne figurent pas dans la source principale. Ils ne sont pas pris en compte dans le recensement des $\mathrm{AO}$, parce qu'il ne s'agit pas à proprement parler d'organismes de recherche.

\section{Académie des technologies}

Lieu de réflexions et de propositions d'actions face aux grands défis technologiques: « Pour un progrès raisonné choisi, partagé ».

\section{$\mathrm{ANR}=$ Agence nationale de la recherche}

Il s'agit d'une agence de financement et non d'un organisme de recherche.

\section{IHEST = Institut des hautes études pour la science et la technologie}

L'IHEST organise des formations et réflexions sur les finalités de la recherche, ses enjeux économiques, sociaux et politiques. Il diffuse ses analyses en vue de construire une culture collective de la recherche et de l'innovation.

\section{A.9 Bibliothèque nationale de France}

Bien que n'étant pas principalement un établissement de recherche, la Bibliothèque nationale de France (BNF) a une activité de recherche.

Portail HAL ouvert le 5 juillet 2012

https://hal-bnf .archives-ouvertes.fr/

D 617 / N 1458

14. https://www.performance-publique.budget.gouv.fr/sites/performance_publique/files/ farandole/ressources/2018/pap/pdf/jaunes/jaune2018_recherche.pdf (Consulté le 30/11/2017), annexe 7 , p. 285 


\section{Annexe B}

\section{Données : recensement de l'OA week 2017}

\section{Méthode de constitution du corpus}

\section{Sources}

http://www.oaweekfrance.org/programme/ [Programme 2017 consulté le 27 octobre 2017]. Ce programme est incomplet. Pour lister tous les événements saisis dans la base après la publication du programme, ajouter date à la fin de l'url, en la faisant varier du 2017-10-11/ au 2017-11-13/. Par exemple pour le 23 octobre 2017 :

http://www . oaweekfrance.org/programme/2017-10-23/

\section{Codage des régions}

ARA Auvergne-Rhône-Alpes

BFC Bourgogne-Franche-Comté

BRE Bretagne

CVL Centre-Val de Loire

COR Corse

GES Grand Est

HDF Hauts-de-France

IDF Île-de-France

NOR Normandie

NAQ Nouvelle-Aquitaine

OCC Occitanie

PDL Pays de la Loire

PAC Provence-Alpes-Côte-d'Azur 


\section{Evénements}

\begin{tabular}{|c|c|c|c|c|c|c|}
\hline Dates & Heures & Événement & Organisateur & Lieu & Type & Région \\
\hline $24 / 10$ & $\begin{array}{l}10 \mathrm{~h} 00 \\
\text { à } \\
11 \mathrm{~h} 00\end{array}$ & IdHalThon & $\begin{array}{l}\text { Inria Grenoble } \\
\text { Rhône Alpes }\end{array}$ & Montbonnot & Ateliers & ARA \\
\hline $24 / 10$ & Journée & $\begin{array}{l}\text { L'open access } \\
\text { révélateur de } \\
\text { synergies } \\
\text { locales }\end{array}$ & $\begin{array}{l}\text { Université de } \\
\text { Lyon }\end{array}$ & $\begin{array}{l}\text { Maison } \\
\text { Internatio- } \\
\text { nale des } \\
\text { Langues et } \\
\text { des } \\
\text { Cultures } \\
\text { (MILC), } \\
\text { Lyon }\end{array}$ & $\begin{array}{l}\text { Atelier, } \\
\text { Conf. }\end{array}$ & $\overline{\mathrm{ARA}}$ \\
\hline $\begin{array}{l}23- \\
27 / 10\end{array}$ & Journée & $\begin{array}{l}\text { Open Access } \\
\text { Week }\end{array}$ & $\begin{array}{l}\text { Arts et } \\
\text { Métiers - } \\
\text { Bibliothèque } \\
\text { du campus de } \\
\text { Cluny }\end{array}$ & Cluny & $\mathrm{NC}$ & $\mathrm{BFC}$ \\
\hline $\begin{array}{l}23- \\
27 / 10\end{array}$ & $\begin{array}{l}13 \mathrm{~h} 00 \\
\text { à } \\
14 \mathrm{~h} 00\end{array}$ & $\begin{array}{l}\text { Café Open } \\
\text { Access }\end{array}$ & $\begin{array}{l}\text { INSA de } \\
\text { Rennes }\end{array}$ & Rennes & Stand & $\mathrm{BRE}$ \\
\hline $24 / 10$ & $\begin{array}{l}9 \mathrm{~h} 30 \mathrm{à} \\
14 \mathrm{~h} 00\end{array}$ & $\begin{array}{l}\text { Open Access } \\
\text { Week - Créez } \\
\text { votre IDHal }\end{array}$ & $\begin{array}{l}\text { Inria Nancy } \\
\text { Grand Est }\end{array}$ & $\begin{array}{l}\text { Villers-les- } \\
\text { Nancy }\end{array}$ & Atelier & GES \\
\hline $\begin{array}{l}23- \\
28 / 10\end{array}$ & Journée & $\begin{array}{l}\text { Expositions } \\
\text { "Les ordis } \\
\text { libres" }\end{array}$ & $\begin{array}{l}\text { Université de } \\
\text { Haute Alsace } \\
\text { (UHA) }\end{array}$ & $\begin{array}{l}\text { SCD, BU } \\
\text { Colmar, } \\
\text { Colmar }\end{array}$ & Stand & GES \\
\hline $\begin{array}{l}23- \\
28 / 10\end{array}$ & Journée & $\begin{array}{l}\text { Exposition "les } \\
\text { ordis libres" }\end{array}$ & $\begin{array}{l}\text { Université de } \\
\text { Haute Alsace } \\
\text { (UHA) }\end{array}$ & $\begin{array}{l}\text { SCD, BU } \\
\text { Fonderie, } \\
\text { Mulhouse }\end{array}$ & Stand & GES \\
\hline $\begin{array}{l}23- \\
28 / 10\end{array}$ & Journée & $\begin{array}{l}\text { Exposition "les } \\
\text { ordis libres" }\end{array}$ & $\begin{array}{l}\text { Université de } \\
\text { Haute Alsace } \\
\text { (UHA) }\end{array}$ & $\begin{array}{l}\text { BU Illberg, } \\
\text { Mulhouse }\end{array}$ & Stand & GES \\
\hline $24 / 10$ & $\begin{array}{l}13 \mathrm{~h} 15 \\
\text { à } \\
14 \mathrm{~h} 00\end{array}$ & Atelier Zotero & $\begin{array}{l}\text { Université de } \\
\text { Haute Alsace }\end{array}$ & $\begin{array}{l}\text { BU Illberg, } \\
\text { Mulhouse }\end{array}$ & Atelier & GES \\
\hline $\begin{array}{l}23- \\
27 / 10\end{array}$ & Journée & $\begin{array}{l}\text { Open Access } \\
\text { Week à } \\
\text { Strasbourg }\end{array}$ & $\begin{array}{l}\text { Université de } \\
\text { Strasbourg } \\
\text { CCN (Centre } \\
\text { de Culture } \\
\text { Numérique), } \\
\text { l'URFIST et } \\
\text { les } \\
\text { bibliothèques } \\
\text { de l'Université } \\
\text { de Strasbourg }\end{array}$ & Strasbourg & $\begin{array}{l}\text { Atelier, } \\
\text { Conf. }\end{array}$ & GES \\
\hline
\end{tabular}




\begin{tabular}{|c|c|c|c|c|c|c|}
\hline Dates & Heures & Événement & Organisateur & Lieu & Type & Région \\
\hline $26 / 10$ & 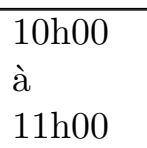 & IdHalThon & $\begin{array}{l}\text { Inria Lille } \\
\text { Nord Europe }\end{array}$ & $\begin{array}{l}\text { Villeneuve- } \\
\text { d'Ascq }\end{array}$ & Atelier & $\mathrm{HDF}$ \\
\hline $27 / 10$ & $\begin{array}{l}11 \mathrm{~h} 00 \\
\text { à } \\
14 \mathrm{~h} 00\end{array}$ & $\begin{array}{l}\text { L'open access } \\
\text { par le jeu }\end{array}$ & $\begin{array}{l}\text { Lilliad - } \\
\text { Learning } \\
\text { Center } \\
\text { Innovation }\end{array}$ & $\begin{array}{l}\text { Villeneuve- } \\
\text { d'Ascq }\end{array}$ & Atelier & $\mathrm{HDF}$ \\
\hline $24 / 10$ & $\begin{array}{l}10 \mathrm{~h} 00 \\
\text { à } \\
13 \mathrm{~h} 00\end{array}$ & $\begin{array}{l}\text { Open Access } \\
\text { Week à la BU } \\
\text { de } \\
\text { Valenciennes }\end{array}$ & $\begin{array}{l}\text { Université de } \\
\text { Valenciennes, } \\
\text { BU du Mont } \\
\text { Houy }\end{array}$ & Valenciennes & Atelier & $\mathrm{HDF}$ \\
\hline $26 / 10$ & $\begin{array}{l}13 \mathrm{~h} 00 \\
\text { à } \\
16 \mathrm{~h} 00\end{array}$ & $\begin{array}{l}\text { Open Access } \\
\text { Week à la BU } \\
\text { de } \\
\text { Valenciennes }\end{array}$ & $\begin{array}{l}\text { Université de } \\
\text { Valenciennes, } \\
\text { BU du Mont } \\
\text { Houy }\end{array}$ & Valenciennes & Atelier & HDF \\
\hline $09 / 11$ & $\begin{array}{l}\begin{array}{l}12 \mathrm{~h} 00 \\
\text { à } \\
14 \mathrm{~h} 00\end{array}\end{array}$ & $\begin{array}{l}\text { L'open access } \\
\text { par le jeu }\end{array}$ & $\begin{array}{l}\text { Université } \\
\text { Lille } 2\end{array}$ & Lille & Atelier & $\mathrm{HDF}$ \\
\hline $24 / 10$ & $\begin{array}{l}9 \mathrm{~h} 30 \text { à } \\
14 \mathrm{~h} 30\end{array}$ & $\begin{array}{l}\text { Open Access } \\
\text { Café }\end{array}$ & $\begin{array}{l}\text { Arts et } \\
\text { Métiers - } \\
\text { Bibliothèque } \\
\text { du campus de } \\
\text { Paris }\end{array}$ & Paris & Atelier & IDF \\
\hline $23 / 10$ & $\begin{array}{l}12 \mathrm{~h} 00 \\
\text { à } \\
14 \mathrm{~h} 00\end{array}$ & $\begin{array}{l}\text { Open Access } \\
\text { Café le } 23 / 10 \\
\text { à la faculté de } \\
\text { pharmacie de } \\
\text { Paris }\end{array}$ & $\begin{array}{l}\text { Pôle } \\
\text { Pharmacie de } \\
\text { la BIU Santé }\end{array}$ & Paris & Stand & IDF \\
\hline $24 / 10$ & $\begin{array}{l}14 \mathrm{~h} 00 \\
\text { à } \\
17 \mathrm{~h} 30\end{array}$ & $\begin{array}{l}\text { Journée } \\
\text { "Accès ouvert" } \\
2017 \text { à la } \\
\text { BULAC }\end{array}$ & $\begin{array}{l}\text { BULAC, } \\
\text { rez-de-jardin, } \\
\text { salle RJ. } 21\end{array}$ & Paris & Atelier & IDF \\
\hline $\begin{array}{l}23- \\
27 / 11\end{array}$ & Journée & $\begin{array}{l}\text { Exposition } \\
\text { Data } \\
\text { Incognita à } \\
\text { l'École des } \\
\text { Ponts } \\
\text { ParisTech } \\
\end{array}$ & $\begin{array}{l}\text { École des } \\
\text { Ponts } \\
\text { ParisTech }\end{array}$ & $\begin{array}{l}\text { Champs- } \\
\text { sur-Marne }\end{array}$ & Stand & IDF \\
\hline $\begin{array}{l}23- \\
27 / 10\end{array}$ & $\begin{array}{l}\text { 9h30 à } \\
19 \mathrm{~h} 00\end{array}$ & $\begin{array}{l}\text { Exposition } \\
\text { open access }\end{array}$ & $\begin{array}{l}\text { École normale } \\
\text { supérieure - } \\
\text { Bibliothèque } \\
\text { de mathéma- } \\
\text { tiques et } \\
\text { Informatique }\end{array}$ & Paris & Stand & IDF \\
\hline
\end{tabular}




\begin{tabular}{|c|c|c|c|c|c|c|}
\hline Dates & Heures & Événement & Organisateur & Lieu & Type & Région \\
\hline $26 / 10$ & $\begin{array}{l}17 \mathrm{~h} 30 \\
\text { à } \\
19 \mathrm{~h} 00\end{array}$ & $\begin{array}{l}\text { Soirée jeu de } \\
\text { l'OA }\end{array}$ & $\begin{array}{l}\text { École normale } \\
\text { supérieure - } \\
\text { Bibliothèque } \\
\text { des sciences } \\
\text { expérimentales }\end{array}$ & Paris & Atelier & IDF \\
\hline $24 / 10$ & $\begin{array}{l}9 \mathrm{~h} 30 \text { à } \\
16 \mathrm{~h} 00\end{array}$ & $\begin{array}{l}\text { Libérez pour } \\
\text { mieux } \\
\text { valoriser : } \\
\text { recherche et } \\
\text { open access }\end{array}$ & $\begin{array}{l}\text { École normale } \\
\text { supérieure } \\
\text { Réseau des } \\
\text { bibliothèques - } \\
\text { Salle des Actes }\end{array}$ & Paris & $\begin{array}{l}\text { Atelier, } \\
\text { Conf. }\end{array}$ & IDF \\
\hline $24 / 10$ & $\begin{array}{l}20 \mathrm{~h} 30 \\
\text { à } \\
23 \mathrm{~h} 00\end{array}$ & $\begin{array}{l}\text { The Internet's } \\
\text { own boy } \\
\text { (documentaire } \\
\text { sur la vie et } \\
\text { les combats } \\
\text { d'Aaron } \\
\text { Swart) }\end{array}$ & $\begin{array}{l}\text { École normale } \\
\text { supérieure, } \\
\text { salle Dussane }\end{array}$ & Paris & Atelier & IDF \\
\hline $26 / 10$ & $\begin{array}{l}12 \mathrm{~h} 00 \\
\text { à } \\
14 \mathrm{~h} 00\end{array}$ & IdHalThon & Inria Paris & Paris & Atelier & IDF \\
\hline $\begin{array}{l}16- \\
27 / 10\end{array}$ & Journée & $\begin{array}{l}\text { Libérer les } \\
\text { publications } \\
\text { scientifiques : } \\
\text { tout savoir sur } \\
\text { l'Open Access }\end{array}$ & $\begin{array}{l}\text { Paris Sciences } \\
\text { et Lettres, } \\
\text { Paris. }{ }^{1}\end{array}$ & Paris & $\begin{array}{l}\text { Atelier, } \\
\text { Conf., } \\
\text { Stand }\end{array}$ & IDF \\
\hline $\begin{array}{l}23- \\
26 / 10\end{array}$ & $\begin{array}{l}15 \mathrm{~h} 00 \\
\text { à } \\
18 \mathrm{~h} 00\end{array}$ & $\begin{array}{l}\text { Atelier Open } \\
\text { Access et } \\
\text { dépôt dans } \\
\text { SPIRE }\end{array}$ & $\begin{array}{l}\text { Sciences Po } \\
\text { Paris, } \\
\text { Bibliothèque } \\
\text { de recherche }\end{array}$ & Paris & Atelier & IDF \\
\hline $\begin{array}{l}11- \\
27 / 10\end{array}$ & $\begin{array}{l}11 \mathrm{~h} 30 \\
- \\
13 \mathrm{~h} 30\end{array}$ & $\begin{array}{l}\text { Cafés ouverts } \\
\text { dans les } \\
\text { laboratoires }\end{array}$ & $\begin{array}{l}\text { Université de } \\
\text { Versailles } \\
\text { Saint-Quentin- } \\
\text { en-Yvelines }\end{array}$ & Versailles & Stand & IDF \\
\hline $\begin{array}{l}23- \\
26 / 10\end{array}$ & $\begin{array}{l}9 \mathrm{~h} 30 \text { à } \\
17 \mathrm{~h} 30\end{array}$ & $\begin{array}{l}\text { Open access } \\
\text { café : penser, } \\
\text { chercher, } \\
\text { publier } \\
\text { autrement à } \\
\text { l'Université } \\
\text { Paris } \\
\text { Descartes }\end{array}$ & $\begin{array}{l}\text { Université } \\
\text { Paris } \\
\text { Descartes }\end{array}$ & Paris & Stand & IDF \\
\hline $\begin{array}{l}23- \\
27 / 10\end{array}$ & $\begin{array}{l}12 \mathrm{~h} 00 \\
\text { à } \\
14 \mathrm{~h} 30\end{array}$ & $\begin{array}{l}\text { Ateliers Open } \\
\text { access \& dépôt } \\
\text { dans HAL }\end{array}$ & $\begin{array}{l}\text { Université } \\
\text { Paris Diderot }\end{array}$ & Paris & Atelier & IDF \\
\hline
\end{tabular}

1. Le programme détaillé mentionne plusieurs événements à l'Université Paris-Dauphine, l'ESPGG, l'ESPCI, l'École normale supérieure, MINES Paris Tech, l'Inria de Paris et l'EHESS. 


\begin{tabular}{|c|c|c|c|c|c|c|}
\hline Dates & Heures & Événement & Organisateur & Lieu & Type & Région \\
\hline $25 / 10$ & $\begin{array}{l}\text { 13h00 } \\
\text { à } \\
17 \mathrm{~h} 00\end{array}$ & $\begin{array}{l}\text { S'approprier } \\
\text { HAL pour } \\
\text { participer à } \\
\text { l'Open Access }\end{array}$ & $\begin{array}{l}\text { Université } \\
\text { Paris Est } \\
\text { Marne la } \\
\text { Vallée, } \\
\text { Bibliothèque } \\
\text { Georges Perec }\end{array}$ & & Atelier & IDF \\
\hline $\begin{array}{l}23- \\
25 / 10\end{array}$ & $\begin{array}{l}11 \mathrm{~h} 45 \\
\text { à } \\
14 \mathrm{~h} 30\end{array}$ & $\begin{array}{l}\text { A la } \\
\text { découverte de } \\
\text { HAL-UPMC } \\
\text { et de l'Open } \\
\text { access }\end{array}$ & $\begin{array}{l}\text { Université } \\
\text { Pierre et } \\
\text { Marie Curie }\end{array}$ & & Stand & IDF \\
\hline $26 / 10$ & $\begin{array}{l}10 \mathrm{~h} 00 \\
\text { à } \\
12 \mathrm{~h} 00\end{array}$ & $\begin{array}{l}\text { Open access } \\
\text { café }\end{array}$ & $\begin{array}{l}\text { Arts et } \\
\text { Métiers - } \\
\text { Bibliothèque } \\
\text { du campus de } \\
\text { Bordeaux- } \\
\text { Talence }\end{array}$ & Talence & Atelier & NAQ \\
\hline $24 / 10$ & $\begin{array}{l}14 \mathrm{~h} 00 \\
\text { à } \\
15 \mathrm{~h} 00\end{array}$ & IdHalThon & $\begin{array}{l}\text { Inria } \\
\text { Bordeaux - } \\
\text { Sud-Ouest }\end{array}$ & Talence & Atelier & NAQ \\
\hline $\begin{array}{l}26 / 10- \\
03 / 11\end{array}$ & Journée & $\begin{array}{l}\text { Open access } \\
\text { Week à } \\
\text { l'université de } \\
\text { Nouvelle- } \\
\text { Calédonie }\end{array}$ & $\begin{array}{l}\text { Université de } \\
\text { Nouvelle- } \\
\text { Calédonie, } \\
\text { Bibliothèque }\end{array}$ & Nouméa & $\begin{array}{l}\text { Atelier, } \\
\text { Table } \\
\text { ronde }\end{array}$ & $\mathrm{NC}$ \\
\hline $\begin{array}{l}23 / 10- \\
15 / 12\end{array}$ & Journée & $\begin{array}{l}\text { Open Action : } \\
\text { l'Open Access } \\
\text { en exemples }\end{array}$ & $\begin{array}{l}\text { COMUE } \\
\text { Languedoc- } \\
\text { Roussillon } \\
\text { Universités. }^{2} \text {. }\end{array}$ & & Stand & $\mathrm{OCC}$ \\
\hline $\begin{array}{l}23- \\
27 / 10\end{array}$ & $\begin{array}{l}9 \mathrm{~h} 30 \text { à } \\
11 \mathrm{~h} 30\end{array}$ & $\begin{array}{l}\text { Café Open } \\
\text { Access }\end{array}$ & $\begin{array}{l}\text { IRD France } \\
\text { Sud, Centre de } \\
\text { Documenta- } \\
\text { tion }\end{array}$ & Montpellier & Stand & $\mathrm{OCC}$ \\
\hline $23 / 10$ & & $\begin{array}{l}\text { Ouverture du } \\
\text { portail HAL } \\
\text { de l'Université } \\
\text { de } \\
\text { Montpellier }{ }^{3}\end{array}$ & $\begin{array}{l}\text { Université de } \\
\text { Montpellier }\end{array}$ & $\mathrm{NC}$ & $\mathrm{NC}$ & $\mathrm{OCC}$ \\
\hline
\end{tabular}

2. Comprend 11 interviews durant lesquelles les personnes interrogées parlent de leur démarche Open Access (enseignants-chercheurs, chercheurs), ou de celle de leurs établissements (gestionnaire de service de recherche, professionnels de l'information et de la documentation...)

3. Aucun lien vers ledit portail ne figure dans e programme. 


\begin{tabular}{|c|c|c|c|c|c|c|}
\hline Dates & Heures & Événement & Organisateur & Lieu & Type & Région \\
\hline $26 / 10$ & $\begin{array}{l}16 \mathrm{~h} 00 \\
\text { à } \\
17 \mathrm{~h} 30\end{array}$ & $\begin{array}{l}\text { Atelier "Vos } \\
\text { travaux } \\
\text { connus dans le } \\
\text { monde entier } \\
\text { grâce à l'open } \\
\text { access" }\end{array}$ & $\begin{array}{l}\text { Université de } \\
\text { Montpellier } \\
\text { BU Lettres } \\
\text { sciences } \\
\text { humaines } \\
\text { Ramon Llull }\end{array}$ & Montpellier & Atelier & $\mathrm{OCC}$ \\
\hline $\begin{array}{l}24 / 10- \\
17 / 11\end{array}$ & & $\begin{array}{l}\text { L'OA week à } \\
\text { l'Université de } \\
\text { Nîmes }\end{array}$ & $\begin{array}{l}\text { Université de } \\
\text { Nîmes (SCD) } \\
\text { et Espace } \\
\text { Recherche du } \\
\text { site des } \\
\text { Carmes }\end{array}$ & Nîmes & Stand & $\mathrm{OCC}$ \\
\hline $\begin{array}{l}13 / 11- \\
24 / 11\end{array}$ & Journée & $\begin{array}{l}\text { Exposition sur } \\
\text { l'OPEN } \\
\text { ACCESS }\end{array}$ & $\begin{array}{l}\text { Université de } \\
\text { Perpignan, BU } \\
\text { Droit-Lettres }\end{array}$ & Perpignan & $\begin{array}{l}\text { Conf., } \\
\text { Stand }\end{array}$ & $\mathrm{OCC}$ \\
\hline $24 / 10$ & $\begin{array}{l}16 \mathrm{~h} 00 \\
\text { à } \\
17 \mathrm{~h} 30\end{array}$ & $\begin{array}{l}\text { Atelier "Vos } \\
\text { travaux } \\
\text { connus dans le } \\
\text { monde entier } \\
\text { grâce à l'open } \\
\text { access" }\end{array}$ & $\begin{array}{l}\text { Université } \\
\text { Paul-Valéry } \\
\text { Montpellier 3, } \\
\text { Site } \\
\text { Saint-Charles } \\
\text { 2, salle } 003 \\
\text { Caryatides }\end{array}$ & Montpellier & Atelier & $\mathrm{OCC}$ \\
\hline $\begin{array}{l}23- \\
27 / 10\end{array}$ & Journée & $\begin{array}{l}\text { Histoires } \\
\text { sonores de la } \\
\text { phonothèque } \\
\text { de la MMSH } \\
\text { libres d'écoute }\end{array}$ & $\begin{array}{l}\text { Aix Marseille } \\
\text { Université }\end{array}$ & & Atelier & $\overline{\mathrm{PAC}}$ \\
\hline $09 / 11$ & $\begin{array}{l}9 \mathrm{~h} 30 \text { à } \\
16 \mathrm{~h} 30\end{array}$ & $\begin{array}{l}\text { Journée Open } \\
\text { Access à AMU }\end{array}$ & $\begin{array}{l}\text { Aix Marseille } \\
\text { Université, } \\
\text { Campus } \\
\text { Schuman } \\
\text { Lettres }\end{array}$ & $\begin{array}{l}\text { Aix-en- } \\
\text { Provence }\end{array}$ & $\begin{array}{l}\text { Atelier, } \\
\text { Conf. }\end{array}$ & PAC \\
\hline $24 / 10$ & $\begin{array}{l}9 \mathrm{~h} 30 \text { à } \\
10 \mathrm{~h} 30\end{array}$ & $\begin{array}{l}\text { Rencontre "Et } \\
\text { si on en } \\
\text { parlait des } \\
\text { archives } \\
\text { ouvertes" }\end{array}$ & $\begin{array}{l}\text { Arts et } \\
\text { Métiers } \\
\text { Bibliothèque } \\
\text { campus d'Aix- } \\
\text { en-Provence }\end{array}$ & $\begin{array}{l}\text { Aix-en- } \\
\text { Provence }\end{array}$ & Atelier & PAC \\
\hline $26 / 10$ & $\begin{array}{l}13 \mathrm{~h} 00 \\
\text { à } \\
14 \mathrm{~h} 00\end{array}$ & IdHalThon & $\begin{array}{l}\text { Inria Sophia } \\
\text { Antipolis } \\
\text { Mediterranée }\end{array}$ & $\begin{array}{l}\text { Sophia } \\
\text { Antipolis }\end{array}$ & Atelier & PAC \\
\hline $\begin{array}{l}23- \\
26 / 10\end{array}$ & $\begin{array}{l}12 \mathrm{~h} 00 \\
\text { à } \\
14 \mathrm{~h} 00\end{array}$ & $\begin{array}{l}\text { Open Access } \\
\text { Week : portes } \\
\text { ouvertes BU }\end{array}$ & $\begin{array}{l}\text { Le Mans } \\
\text { Université }\end{array}$ & Le Mans & Stand & PDL \\
\hline
\end{tabular}




\section{Annexe C}

\section{Extrait des documents stratégiques de l'Enssib}

Les mises en gras ont été ajoutées pour les besoins du présent rapport.

\section{C.1 Contrat de site 2016-2020 Université de Lyon : volet spécifique Enssib}

[pp. 429-430]

V. Valoriser et diffuser la production scientifique des savoirs

Acteur reconnu par les professionnels des bibliothèques, l'Enssib a pour objectif d'inscrire sa politique de valorisation dans une cartographie nationale. Elle ambitionne ainsi de :

- faire du site web de l'école une véritable plateforme nationale et internationale de ressources, pour stimuler la recherche professionnelle et les échanges ;

- développer et rendre encore plus visible sa bibliothèque numérique et ses ressources en ligne;

- développer fortement les partenariats en termes de publications et de manifestations (coédition en particulier, inscription dans les grands projets de valorisation des publications numériques);

- être force de proposition dans la mise en place de la politique documentaire de l'UdL.

\section{C.2 Projet d'établissement Enssib 2020}

[p. 3]

I - Orientations stratégiques du projet Enssib 2020

Le projet Enssib 2020 comporte trois orientations majeures à caractère transversal permettant d'ancrer la stratégie et la gouvernance de l'Ecole :

A - Inscrire le projet de l'école dans la stratégie du site lyonnais en cohérence avec ses missions nationales d'école d'application et sa position d'établissement de référence pour la communauté professionnelle des bibliothèques et de la documentation.

B - Développer et formaliser une politique volontariste et ambitieuse de partenariats. 
$[\ldots]$

I - Objectifs stratégiques et opérationnels du projet Enssib 2020

$[\ldots]$

- Valoriser et diffuser la production des savoirs

- Améliorer le pilotage, optimiser les moyens

$[\ldots]$

[p. 7]

- Identifier et valoriser, à destination des publics de l'école, les travaux de recherche, les appels à communications ou à projets relevant des thématiques scientifiques de l'Enssib. L'activité de veille scientifique réalisée à l'Enssib couvre l'ensemble des champs de l'école. Elle est menée par différents services en fonction des thématiques et des types d'information visés. L'information relative aux activités de recherche est aujourd'hui rassemblée par différents acteurs (BBF, EnssibLab, Presses de l'Enssib, Revue de l'Enssib, Relations internationales...). L'enjeu est de structurer l'activité de veille pour mieuc en faire bénéficier l'ensemble des publics de l'école, mais aussi de faire de cette veille un levier d'émergence de projets regroupant des chercheurs et des professionnels. Cette veille informationnelle fera également l'objet d'un projet de développement web spécifique, dans le cadre d'une collaboration active avec les plateformes nationales implantées sur le site (CCSD, Persée).

- Développer le rôle d'observatoire des pratiques et usages

$[\ldots]$

[p. 10]

- Mieux valoriser la production scientifique de l'école, de ses chercheurs, de ses personnels scientifiques, des étudiants se dirigeant vers la recherche, des élèves et de l'ensemble de ses partenaires dans la communauté professionnelle (EnssibLab, carnet DLIS, Revue de l'Enssib, mise en valeur des travaux versés dans la bibliothèque numérique, etc.)

- Développer et rendre encore plus visibles la bibliothèque numérique et les ressources en ligne de l'école. L'Enssib renforcera ses partenariats avec d'autres institutions pour mettre en oeuvre une politique de développement et d'enrichissement de sa bibliothèque numérique.

- Faire du site web de l'école une véritable plateforme nationale et internationale de ressources librement accessibles pour stimuler la recherche professionnelle et les échanges. L'Enssib procèdera à une refonte de son site web. Elle mettra en oeuvre un projet multilingue, innovant, pour valoriser son projet de développement, sa démarche partenariale, son ouverture internationale, la richesse de ses ressources et de ses services, dans une approche résolument ouverte.

- Développer fortement les partenariats en termes de publications et de manifestations, inscrire la programmation sur l'ensemble du territoire. Développer la production éditoriale des Presses de l'Enssib, vers une offre hybride (papier et numérique) innovante 


\section{Glossaire}

ANR Agence nationale de la recherche. L'ANR est une agence de moyens.

AO Archive ouverte.

AOI Archive ouverte institutionnelle.

APC Article processing charge. Les APC sont des frais de traitement payés par l'institution employant un chercheur pour que son article soit publiés en accès ouvert.

CDEFI Conférence des directeurs des écoles françaises d'ingénieurs.

CGE Conférence des grandes écoles.

CNU Conseil national des universités. Le CNU est organisé en sections, correspondant chacune à une discipline.

CoSO Comité pour la Science Ouverte. Le CoSO est issu de la transformation, en 2018, de la Bibliothèque scientifique numérique (BSN).

CPU Conférence des présidents d'université.

Enssib École nationale supérieure des sciences de l'information et des bibliothèques.

Epi-revue Revue en OA avec comité de lecture créée à partir de preprints déposés dans un dépôt d'archives ouvertes.

ESR Enseignement supérieur et recherche

Gold OA ou voie dorée. Développement de l'OA par la publication de revues en $\mathrm{OA}$, très souvent conditionné au paiement d'APC.

Green OA ou voie verte. Développement de l'OA par le dépôt par les chercheurs d'articles dans des archives ouvertes.

HAL Hyper articles en ligne. L'archive ouverte pluridisciplinaire HAL, est destinée au dépôt et à la diffusion d'articles scientifiques de niveau recherche, publiés ou non, et de thèses, émanant des établissements d'enseignement et de recherche français ou étrangers, des laboratoires publics ou privés. Elle est développée par le Centre de communication scientifique directe (CCSD) du CNRS.

HCERES Haut Conseil de l'évaluation de la recherche et de l'enseignement supérieur

IST Information scientifique et technique

Mégarevue Revue en Gold OA publiant exclusivement en ligne des articles dans de nombreuses disciplines. 
MESR / MESRI Ministère de l'Enseignement supérieur et de la Recherche / Ministère de l'Enseignement supérieur, de la Recherche et de l'Innovation

OA Open Access, ou, plus rarement, Open Archive

Revue hybride Revue diffusée sur abonnement permettant cependant de publier aussi des articles en OA, moyennant le paiement d'APC.

Revue prédatrice Revue en Gold OA, sans garantie ni notoriété scientifiques, créée uniquement dans le but d'encaisser des APC.

SCD Service commun de la documentation. Il s'agit du service universitaire qui gère les bibliothèques universitaires (BU) et les bibliothèques associées.

URFIST Unité Régionale de Formation à l'Information Scientifique et Technique. 


\section{Bibliographie}

Les références listées dans la bibliographie ont toutes été utilisée dans le présent rapport. On pourra compléter en se référant à la page de ressources commentées que Frédéric Hélein, Directeur scientifique du Réseau National des Bibliothèques de Mathématiques, tient à jour :

https://webusers.imj-prg.fr/ frederic.helein/editio.html

$$
\text { *** }
$$

7 bonnes raisons de déposer dans l'archive ouverte HAL. 2 mai 2016. URL : https:// openaccess . univ-rennes1.fr/7-bonnes-raisons-de-deposer-dans-larchiveouverte-hal (visité le 04/04/2018) (cf. p. 58).

Academia.edu. URL : https://www . academia.edu/ (visité le 12/03/2018) (cf. p. 40,61).

ADEB. OPEN ACCESS : Un projet de décret sans concertation des parties prenantes menace l'édition scientifique. $1^{\mathrm{er}}$ mar. 2018. URL : http://adeb . be/sites/502a 034d9f83256166000004 / assets / 5a9954031dd64768af000964 / Communiqu _ _de _ presse___OA.pdf (visité le 23/04/2018) (cf. p. 29).

Agrixiv. URL : https://agrixiv.org/ (visité le 23/04/2018) (cf. p. 27).

Altmetrics : a manifesto. URL : http : //altmetrics . org/manifesto/ (visité le 22/03/2018) (cf. p. 44).

AMI pour la constitution du Comité pour la Science Ouverte (CoSO). URL : http: //www .bibliothequescientifiquenumerique.fr/ami-pour-la-constitutiondu-comite-pour-la-science-ouverte-coso/ (visité le 26/03/2018) (cf. p. 45, 70, $71)$.

An International Perspective on Open Access in Scholarly Communication : Achievements and Challenges / Une perspective internationale sur le libre accès dans la communication scientifique : succès et défis. 26 avr. 2018. URL : http://www.issn.org/ wp-content/uploads/2018/04/ISSN_UNESCO_PROGRAMME_CONFERENCE_201804261.pdf (visité le 30/04/2018) (cf. p. 56).

ANGLADA, Lluis. "L'accès ouvert en Espagne : mandat, état des lieux et approche progressive actuelle ». JSO 2018. 23 jan. 2018. URL : https://jso2018.sciencesc onf.org/data/pages/Lluis_Anglada_2.pdf (visité le 19/02/2018) (cf. p. 29).

Appel de Jussieu pour la Science ouverte et la bibliodiversité. 10 oct. 2017. URL : http: //jussieucall.org/index-FR.html (visité le 19/04/2018) (cf. p. 21, 23).

Archive ouverte HAL. URL : https://hal . archives-ouvertes.fr/ (cf. p. 25). arXiv.org e-Print archive. URL : https://arxiv.org/ (visité le 19/02/2018) (cf. p. 25). ASAPbio / Accelerating Science and Publication in Biology. URL : http://asapbio.org/ (visité le 22/03/2018) (cf. p. 43).

AURéHAL : Accès Unifié aux Référentiels HAL. URL : https://aurehal.archivesouvertes.fr/ (visité le 12/04/2018) (cf. p. 73). 
Becking, Koen. «Disruptive innovation in scholarly communications ». JSO 2018. 23 jan. 2018. URL : https : // jso2018. sciencesconf .org/data/pages/Koen _ Becking_2.pdf (visité le 20/02/2018) (cf. p. 33).

Benech, Christophe. Protection et propriété des données sur Academia.edu et ResearchGate. 14 mar. 2014. URL : https://archeorient.hypotheses.org/2554 (visité le 31/03/2018) (cf. p. 61).

Berlin Declaration. 22 oct. 2003. URL : https://openaccess.mpg.de/Berlin-Declara tion (visité le 20/04/2018) (cf. p. 22).

BERThAud, Christine et al. «EPISCIENCES - an overlay publication platform ». In : Let's Put Data to Use : Digital Scholarship for the Next Generation. 18th International Conference on Electronic Publishing. Thessaloniki, Greece, 2014, p. 78-87. DOI : 10.3233/978-1-61499-409-1-78. (Visité le 09/03/2018) (cf. p. 39).

Bethesda Statement on Open Access Publishing. 11 avr. 2003. URL : http://legacy. earlham.edu/ peters/fos/bethesda.htm (visité le 20/04/2018) (cf. p. 21).

Bibliothèques et chercheurs : la science ouverte en commun. URL : https : // oawlyon 2018. sciencesconf.org/ (visité le 10/10/2018) (cf. p. 56).

bioRxiv.org - the preprint server for Biology. URL : https://www.biorxiv.org/ (visité le 20/02/2018) (cf. p. 27).

Bohannon, John. «Who's downloading pirated papers? Everyone ». In : Science (25 avr. 2016). DOI : 10.1126/science.aaf5664. URL : http://www.sciencemag.org/news/ 2016/04/whos-downloading-pirated-papers-everyone (visité le 12/03/2018) (cf. p. 42$)$.

Bouchard, Aline. "Nous n'avons pas d'autre choix ": les éditeurs, ResearchGate et les guerres du copyright. 6 nov. 2017. URL : https://urfistinfo.hypotheses.org/ 3126 (visité le 16/04/2018) (cf. p. 40).

- Éléments de comparaison archives ouvertes (HAL) et réseaux sociaux académiques (Academia, Research Gate). Nov. 2016. URL : http://urfist.chartes.psl.eu/ sites/default/files/ab/Bouchard_Comparaison_AO_RSX_112016.pdf (visité le 30/03/2018) (cf. p. 61).

- Pour une utilisation critique des réseaux sociaux académiques. 14 fév. 2014. URL : https://urfistinfo.hypotheses.org/2596 (visité le 31/03/2018) (cf. p. 61).

Budapest Open Access Initiative. 14 fév. 2002. URL : http://www.budapestopenaccess initiative.org/read (visité le 20/04/2018) (cf. p. 21).

CABANAC, Guillaume. " Bibliogifts in LibGen? A study of a text-sharing platform driven by biblioleaks and crowdsourcing ». In : Journal of the Association for Information Science and Technology 67.4 (2016), p. 874-884. ISSN : 2330-1643. DOI : 10.1002/ asi.23445. URL: http://dx.doi.org/10.1002/asi.23445 (cf. p. 43).

Cahiers du CELEC. URL : http : / / cahierscelec.ish-lyon . cnrs . fr/ (visité le 08/03/2018) (cf. p. 37).

CARACO, Benjamin. La voie diamantée du libre accès. Le comptoir de l'accès ouvert Open access desk. 27 jan. 2014. URL : https://oadesk.hypotheses.org/author/ caraco (visité le 01/03/2018) (cf. p. 34).

CasuHAL : club utilisateur. URL : https : / / iww. inria.fr / casuhal/ (visité le 24/04/2018) (cf. p. 30).

CCSD. Les temps forts. URL : https : //www . ccsd.cnrs . fr/presentation/lestemps-forts/ (visité le 19/02/2018) (cf. p. 25).

Centre pour l'édition électronique ouverte. URL : http: / / cleo.openedition .org/ (visité le 08/03/2018) (cf. p. 34). 
Chartron, Ghislaine. «Open Access : origine, enjeux, actualités ». 11 fév. 2013. URL : http://www . openaccess-shs. info/wp-content/uploads/2012/12/Pr\%C3\%A9se ntation-de-Ghislaine-Chartron-11-f\%C3\%A9vrier.pdf (visité le 19/04/2018) (cf. p. 25).

ChemArxiv Repository. URL : http://chemarxiv.org/ (visité le 20/02/2018) (cf.p. 27).

Chercheur / LILLIAD. URL : https://lilliad.univ-lille.fr/chercheur (visité le 28/03/2018) (cf. p. 53).

CNRS. " Résultats 2014 des grands éditeurs scientifiques : une croissance satisfaisante, des profits record. Un terrain favorable à de nouvelles concentrations? $»$ In : DISTinfo 14 (mar. 2015), p. 8. URL : http://www.cnrs.fr/dist/z-outils/documents/ Distinfo2/Distinf14.pdf (cf. p. 19).

CNRS, Conseil scientifique de l'institut des mathématiques (INSMI). Recommandation au sujet des frais de publication (APC). 15 juin 2016. URL : http://www.cnrs.fr/ comitenational/doc/recommandations/2016/Recommandation-csi-INSMI-ausujet-des-frais-de-publication-(APC) .pdf (visité le 08/03/2018) (cf. p. 31).

CNU, Section 60. Recommandations et critères pour les promotions en section 60 . URL : http : //www . cpcnu.fr/web/section-60/conseils-generaux1 (visité le 11/04/2018) (cf. p. 68).

COAR Next Generation Repositories : Vision and Objectives. URL : http://ngr.coarrepositories.org/ (visité le 06/06/2018) (cf. p. 28).

Code de la recherche - Article L533-4. URL : https://www.legifrance.gouv.fr/ affichCodeArticle.do?idArticle=LEGIARTI000033205794\&cidTexte=LEGITEXT 000006071190 (visité le 20/02/2018) (cf. p. 30).

Commission européenne. Pour un meilleur accès aux informations scientifiques : dynamiser les avantages des investissements publics dans le domaine de la recherche. 17 juil. 2012. URL : http://ec.europa.eu/research/science-society/document_ library/pdf_06/era-communication-towards-better-access-to-scientificinformation_fr.pdf (visité le 19/04/2018) (cf. p. 23).

Confederation of Open Access Repositories (COAR) - Towards a global knowledge commons. URL : https : //www . coar-repositories . org/ (visité le 31/05/2018) (cf. p. 28).

Convention de partenariat en faveur des archives ouvertes et de la plateforme mutualisée HAL. 2 avr. 2013. URL : http://cache.media.enseignementsup-recherche.gouv . $\mathrm{fr} / \mathrm{file} / \mathrm{HAL} / 93 / 3 / 01$ _Convention_HAL_246933.pdf (visité le 11/04/2018) (cf. p. 69).

CORE. URL : https://core.ac.uk/ (visité le 06/06/2018) (cf. p. 28).

COSNARD, Michel. «L'évaluation au Hcéres ». JSO 2018. Paris. URL : https : // jso2018. sciencesconf.org/data/pages/Michel_Cosnard_2.pdf (cf. p. 69).

CoSO. Ouvrir la Science. URL : https : / / www . ouvrirlascience.fr/ (visité le 10/12/2018) (cf. p. 4, 75).

Couperin ne renouvelle pas l'accord national passé avec Springer. 3 avr. 2018. URL : http : / / www . couperin . org/breves / 1333-couperin-ne-renouvelle-pas-1accord-national-passe-avec-springer (visité le 19/04/2018) (cf. p. 20).

Dakar Declaration on Open Access Publishing in Africa and the Global South / CODESRIA. $1^{\mathrm{er}}$ avr. 2016. URL : http://www.codesria.org/spip.php?article2595 (visité le 27/04/2018) (cf. p. 23).

Data Gueule. Privés de savoir? \#DATAGUEULE 63. 17 oct. 2016. uRl : https : //www . youtube. com/watch?v=WnxqoP-c0ZE (visité le 19/02/2018) (cf. p. 23). 
Déclaration commune des trois conférences (CPU, CDEFI et CGE) au Journées Science ouverte 2018. Jan. 2018. URL : https://jso2018. sciencesconf .org/data/pages/ 20180122_JS02018_TroisConferences_VF_2.pdf (visité le 11/04/2018) (cf. p. 70).

Déclaration d'EBLIDA - Libre accès à l'information scientifique et technique. Avr. 2005. URL : http: //openaccess . inist.fr/?Declaration-d-EBLIDA (visité le 20/04/2018) (cf. p. 22).

Déclaration de l'Alhambra sur le Libre Accès - Libre accès à l'information scientifique et technique. 14 mai 2010. URL : http://openaccess.inist.fr/?Declaration-de1-Alhambra-sur-le (visité le 20/04/2018) (cf. p. 22).

Déclaration de l'IFLA sur le libre accès à la littérature scientifique et aux documents de la recherche - Libre accès à l'information scientifique et technique. 5 déc. 2003. URL : http: //openaccess.inist.fr/?Declaration-de-1-IFLA-sur-le-libre (visité le 20/04/2018) (cf. p. 22).

Declaration de Mexico : A favor del Ecosistema latinoamericano de Acceso Abierto No Comercial. URL : http : / / www . accesoabiertoalyc .org/declaracion-mexico/ (visité le 19/02/2018) (cf. p. 23).

Déclaration de Valparaiso pour une meilleure communication scientifique sur support électronique - Libre accès à l'information scientifique et technique. 15 jan. 2004. URL : http://openaccess.inist.fr/?Declaration-de-Valparaiso-pour-une (visité le 20/04/2018) (cf. p. 22).

Déclaration écossaise sur le Libre Accès - Libre accès à l'information scientifique et technique. 11 oct. 2004. URL : http : // openaccess . inist . fr/?Declarationecossaise-sur-le-Libre (visité le 20/04/2018) (cf. p. 22).

Décret no 92-70 du 16 janvier 1992 relatif au Conseil national des universités. 16 jan. 1992. URL : https : / / www . legifrance . gouv . fr / affichTexte . do ? cidTexte= JORFTEXT000000344860 (visité le 19/02/2018) (cf. p. 68).

Delhi Declaration on Open Access. 14 fév. 2018. URL : http://openaccessindia.org/ delhi-declaration-on-open-access/ (visité le 24/04/2018) (cf. p. 23).

Délibération $n^{\circ} 6$ du conseil d'administration de l'Université Savoie Mont Blanc du 21 novembre 2017 : Dépôt des publications dans HAL. 30 nov. 2017 (cf. p. 52).

Des extensions de navigateurs pour repérer les articles en libre-accès - BU Lyon 1 Université Lyon 1. BU Lyon 1. URL : https ://portaildoc . univ-lyon1.fr/ chercheur/des-extensions-de-navigateurs-pour-reperer-les-articles-enlibre-acces-944526.kjsp (visité le 06/12/2018) (cf. p. 41, 61).

DI-fusion. URL : http://difusion.ulb.ac.be/ (visité le 20/02/2018) (cf. p. 28).

DI-fusion: Guide du déposant. URL : https://www.bib.ulb.ac.be/fr/bibliothequeelectronique/depot-institutionnel-di-fusion/guide-du-deposant/index . html (visité le 20/02/2018) (cf. p. 29).

DIAL. URL : https://dial.uclouvain.be/Home/ (visité le 20/02/2018) (cf. p. 28).

Directory of Open Access Journals. URL : https ://doaj.org (visité le 30/04/2018) (cf. p. 30).

Dissemin. URL : https://dissem.in/ (visité le 05/04/2018) (cf. p. 60).

DoRANum - Données de la recherche : Apprentissage Numérique. URL : http://doran um.fr/ (visité le 19/02/2018) (cf. p. 44).

eartharxiv. URL : https://eartharxiv.org/ (visité le 23/04/2018) (cf. p. 27).

Ecole des Ponts ParisTech : Mandat pour le partage de la science. 27 fév. 2017. URL : https://espacechercheurs.enpc.fr/sites/default/files/MandatOA_Ecolede sPonts.pdf (visité le 23/03/2018) (cf. p. 51). 
EconPapers. URL : https://econpapers.repec.org/ (visité le 23/04/2018) (cf. p. 27).

Edition: "La licence nationale imposée à tous est inacceptable " (M. Bernard, Clermont Auvergne). Newstank education. Article 133695. 19 nov. 2018. uRL : https://edu cation . newstank . fr/fr/tour/news / 133695 / edition-licence-nationaleimposee-tous-inacceptable-bernard-clermont.html (visité le 05/12/2018) (cf. p. 20).

EDP Open. URL : https://www.edp-open.org/ (cf. p. 36).

EDP Sciences. URL : https ://www.edpsciences.org/fr/ (visité le 25/04/2018) (cf. p. 35).

EDP Sciences - French academics to benefit from national open access deal with EDP Sciences. 12 déc. 2017. URL : https : //www . edpsciences . org/fr/actualites/ 1645 - french - academics - to - benefit - from - national - open - access - deal with-edp-sciences (visité le 27/04/2018) (cf. p. 35).

EDP Sciences - National open access deal in France. URL : https://www.edpsciences. org/fr/national-open-access-deal-in-france (visité le 25/04/2018) (cf. p. 35).

En solidarité avec Library Genesis et Sci-Hub. URL : http://custodians . online (visité le 19/02/2018) (cf. p. 43).

engrXiv. URL : https://engrxiv.org/ (visité le 23/04/2018) (cf. p. 27).

Enquête Archives Ouvertes COUPERIN 2017: Résultats de l'enquête. Oct. 2017. URL : https : / / www . couperin . org / images / stories / AO / Depouillement_enquete _ A02017_FINAL_V7.pdf (visité le 30/04/2018) (cf. p. 47).

Epi-revues. URL : https : //www.ccsd.cnrs.fr/epi-revues/ (visité le 19/02/2018) (cf. p. 39).

Episciences. URL : https://www.episciences.org/ (visité le 19/02/2018) (cf. p. 39).

EPRIST. Résultats financiers 2015 de l'édition scientifique. 30 mar. 2016. URL : http: //www.eprist.fr/wp-content/uploads/2016/03/I-IST_16_R\%C3\%A9sultatsFin anciers2015EditionScientifique.pdf (visité le 19/04/2018) (cf. p. 20).

«ERC Scientific Council Statement on Open Access ». In : (déc. 2006), p. 1 (cf. p. 22).

Érudit. URL : http://www.erudit.org/fr/ (visité le 25/04/2018) (cf. p. 36).

Érudit : Accès au contenu. URL : https://apropos.erudit.org/fr/bibliotheques/ acces-au-contenu/ (visité le 25/04/2018) (cf. p. 36).

Fair Open Access. URL : https : //www . fairopenaccess .org/ (visité le 19/02/2018) (cf. p. 38).

FAQ sur la loi République numérique - Open Access France. URL : http://openaccess. couperin.org/category/faq/ (visité le 05/04/2018) (cf. p. 59).

Finch, Janet. Accessibility, sustainability, excellence : how to expand access to research publications. Juin 2012. URL : https ://www.acu.ac.uk/research-informationnetwork/finch-report-final (visité le 20/02/2018) (cf. p. 32).

FinELib's Elsevier agreement and transparency - questions and answers. 2 fév. 2018. URL : http://finelib.fi/finelibs-elsevier-agreement-and-transparencyquestions-and-answers/ (visité le 08/03/2018) (cf. p. 33).

FOSTER. URL : https : //www. fosteropenscience.eu/ (visité le 22/03/2018) (cf. p. 43).

Fovet-RAвот, Cécile. Eviter les éditeurs prédateurs. 2017. URL : http://coop-ist. cirad.fr/editeurs-predateurs (visité le 09/03/2018) (cf. p. 40,62).

Global Open Access Portal (GOAP). United Nations Educational, Scientific and Cultural Organization. URL : http : / / www . unesco . org / new / en / communication - and information/portals-and-platforms/goap/ (visité le 19/04/2018) (cf. p. 25). 
GouzI, Françoise. Academia.edu, ResearchGate... à utiliser avec modération! Toul'AO. 29 jan. 2015. URL : http: //openarchiv. hypotheses.org/2582 (visité le 19/02/2018) (cf. p. 61).

- Comment financer l'Open Access? Un exemple local. Toul'AO. 12 mar. 2018. URL : https://openarchiv.hypotheses.org/4461 (visité le 16/04/2018) (cf. p. 54).

Guide du déposant : Guide d'utilisation de l'archive ouverte SAM à l'attention des chercheurs ENSAM. SAM : Science Arts \& Métiers. Version 2.1. 8 juil. 2013. URL : http://sam.ensam.eu/help.html (visité le 23/03/2018) (cf. p. 51).

HAL : Les portails de l'archive. URL : https://hal.archives-ouvertes.fr/browse/ portal (visité le 17/01/2018) (cf. p. 27).

Hammeau, Thérèse. Les mégarevues. 20 fév. 2014. URL : http://openaccess inist. fr/?Les-megarevues (visité le 09/03/2018) (cf. p. 39).

Héloïse - Politiques des éditeurs en matière de dépôt des articles. URL : https://heloi se.ccsd.cnrs.fr/ (visité le 19/02/2018) (cf. p. 59).

Huet, Sylvestre. Open access : du rêve au cauchemar. 4 oct. 2013. URL : http : // sciences . blogs . liberation . fr / 2013/10/04/open-access-du-reve-aucauchemar-/ (visité le 09/03/2018) (cf. p. 40).

- Open access : du rêve au cauchemar? L'avis de J-C Guédon. 4 oct. 2013. URL : http://sciences.blogs.liberation.fr/2013/10/04/open-access-du-reveau-cauchemar-bis/ (visité le 09/03/2018) (cf. p. 40).

Hybrid open access journal. In : Wikipedia. Page Version ID : 817351083. 27 déc. 2017. URL : https ://en.wikipedia.org/w/index . php?title=Hybrid_open_access_ journal\&oldid=817351083 (visité le 20/02/2018) (cf. p. 31).

IAP. IAP Statement on Access to Scientific Information. 4 déc. 2003. URL : http: / / interacademies . org / 13916 / IAP - Statement - on-Access-to-ScientificInformation (visité le 20/04/2018) (cf. p. 22).

ICanHazPDF. In : Wikipedia. Page Version ID : 805921107. 18 oct. 2017. URL : https: //en.wikipedia.org/w/index.php?title=ICanHazPDF\&oldid=805921107 (visité le 19/02/2018) (cf. p. 42).

Initiative for Open Citations. URL : https://i4oc.org/ (cf. p. 44).

INRIA. Inria défend l'Open Access. 6 nov. 2015. URL : https://www.inria.fr/actu alite/actualites-inria/inria-defend-1-open-access (visité le 23/03/2018) (cf. p. 51).

Institut Pasteur - Médiathèque. URL : https://webext.pasteur.fr/biblio/ressourc es/oai/ (visité le 23/03/2018) (cf. p. 51).

JefFerson, Thomas. To Isaac McPherson. 13 août 1813. URL : http://press-pubs. uchicago.edu/founders/documents/a1_8_8s12.html (visité le 18/04/2018) (cf. p. 21).

Jonchère, Laurent. " Projet d'archive ouverte Université de Rennes 1 : Synthèse sur les politiques institutionnelles de libre accès à la recherche ». In : (2013), p. 36. URL : https://archivesic.ccsd.cnrs.fr/sic_00794497v2 (cf. p. 51).

Journée d'étude Open Access LR "Être vu pour être lu" - Open Access LR. URL : https: //www . open-access . fr/actualites/41-journee-d-etude-open-access-lretre-vu-pour-etre-lu (visité le 06/12/2018) (cf. p. 56).

Journées OA Couperin. 2005. URL : http://openaccess .couperin.org/category/ nos_evenements/joa/ (visité le 30/04/2018) (cf. p. 55).

Kopernio. URL : https://kopernio.com (visité le 18/10/2018) (cf. p. 41). 
L'ANR et l'Open access. URL : http : / / www . agence-nationale-recherche.fr / missions-et-organisation/open-access/ (visité le 11/04/2018) (cf. p. 69).

L'ANR incite les chercheurs à intégrer leurs publications dans le système d'archives ouvertes. URL : http : / www . agence-nationale-recherche.fr/informations / actualites/detail/?tx_ttnews\%5btt_news\%5d=159 (visité le 23/03/2018) (cf. p. 51).

L'ANR, signataire de la Déclaration de San Francisco sur l'évaluation de la recherche. ANR. URL : http : / / www . agence-nationale-recherche.fr/informations / actualites/detail/lanr-signataire-de-la-declaration-de-san-franciscosur-levaluation-de-la-recherche/ (visité le 06/12/2018) (cf. p. 69).

L'open access révélateur de synergies locales. 24 oct. 2017. URL : https://oawlyon2017. sciencesconf .org/ (visité le 19/02/2018) (cf. p. 55).

L'Université de Lorraine s'engage en faveur de la science ouverte / Factuel. 19 avr. 2018. URL : http://factuel. univ-lorraine.fr/node/8472 (visité le 23/04/2018) (cf. p. 54).

La politique open access de Rennes 1.9 juin 2016. URL : https://openaccess.univrennes1.fr/la-politique-open-access-de-rennes-1 (visité le 28/03/2018) (cf. p. 52).

Larousserie, David. "Marin Dacos, héraut de la science ouverte ». In : Le Monde (4 déc. 2018). URL : https ://www.lemonde.fr/sciences/article/2018/12/04/ marin-dacos-heraut-de-la-science-ouverte_5392481_1650684.html (visité le 05/12/2018) (cf. p. 3, 44).

LATrIVE, Florent et Lawrence Lessig. Du bon usage de la piraterie culture libre, sciences ouvertes. Essais. Paris : Exils éd., 2004. 1 vol. (170 p.) ISBN : 2-912969-59-X (cf. p. 21).

LawArXiv. URL : https://osf .io/preprints/lawarxiv (visité le 23/04/2018) (cf. p. 27).

Lazy Scholar. URL : http://www. lazyscholar.org/ (visité le 25/04/2018) (cf. p. 41).

Le (bon) plan S. 4 sept. 2018. URL : https://marlenescorner.net/2018/09/04/lebon-plan-s/ (visité le 07/09/2018) (cf. p. 67).

Le CNRS, Inria et une vingtaine d'établissements d'enseignement supérieur arrêtent leurs abonnements au groupe Springer. AEF info. URL: https://www.aefinfo.fr/ depeche/594873 (visité le 05/12/2018) (cf. p. 20).

Le libre accès. Centre pour l'édition électronique ouverte. URL : http://cleo.openedi tion.org/missions/libre-acces (visité le 08/03/2018) (cf. p. 35).

Le libre accès aux publications et aux données de recherche - Horizon 2020. URL : http: //www . horizon2020 gouv . fr/cid82025/le-libre-acces-aux-publicationsaux-donnees-recherche.html (visité le 06/04/2018) (cf. p. 67).

Le peer review à l'ère de l'open science : enjeux et évolutions. 27 mar. 2018. URL : http: //weburfist . univ-bordeaux.fr/le-peer-review-a-lere-de-lopenscience-enjeux-et-evolutions/ (visité le 16/04/2018) (cf. p. 56).

Les grandes dates. Centre pour l'édition électronique ouverte. URL : http:// cleo . openedition.org/presentation/dates (visité le 08/03/2018) (cf. p. 34).

Les premières journées de la Science Ouverte. URL : https://jnso2018. sciencesconf. org/ (visité le 16/10/2018) (cf. p. 56).

Ley 14/2011, de 1 de junio, de la Ciencia, la Tecnología y la Innovación. $1^{\mathrm{er}}$ juin 2011. URL : http://www.boe.es/buscar/pdf/2011/BOE-A-2011-9617-consolidado. pdf (visité le 19/02/2018) (cf. p. 29). 
Library Genesis. In : Wikipédia. Page Version ID : 141911763. 25 oct. 2017. URL : https: //fr.wikipedia.org/w/index.php?title=Library_Genesis\&oldid=141911763 (visité le 19/02/2018) (cf. p. 42).

Library Genesis / LibGen - The Meta Library. URL : https://sites .google.com/ site/themetalibrary/library-genesis (visité le 19/02/2018) (cf. p. 43).

Library Genesis 2M. URL : http://gen.lib.rus.ec/ (visité le 19/02/2018) (cf. p. 42).

LiÈGE, Université de. Extrait du procès-verbal de la séance du 23 mai 2007 du Conseil d'administration. URL : https://orbi.uliege.be/files/extrait_moniteur_CA. pdf (visité le 20/02/2018) (cf. p. 28).

Ling OA. URL : https://www.lingoa.eu/ (visité le 19/02/2018) (cf. p. 38).

LISSA : Library and information science Scholarship Archive Preprints. URL : https: //osf .io/preprints/lissa (visité le 23/04/2018) (cf. p. 27).

Liste des sections $C N U$ - Portail. URL : http://www.cpcnu.fr/listes-des-sectionscnu (visité le 11/04/2018) (cf. p. 68).

Louvigny, A. Publications scientifiques en accès libre: la mentalité des chercheurs doit aussi évoluer. 19 mar. 2018. URL : https : //www.rtbf .be/info/societe/ detail_publications-scientifiques-en-acces-libre-la-mentalite-deschercheurs-doit-aussi-evoluer?id=9871133 (visité le 29/03/2018) (cf. p. 29).

Lutz, Jean-François. « Dépenses documentaires en BU : de l'euphorie aux questionnements existentiels ». Journées RNBM - Accès ouvert, rêve ou réalité? 18 oct. 2017. URL : http://www.rnbm.org/supports_anf/cirm2017/Presentation_LUTZ_RNBM. pdf (visité le 19/04/2018) (cf. p. 20).

Magron, Agnès. Catégorie "Episciences". URL : https://www.ccsd.cnrs.fr/categor y/episciences/ (visité le 09/03/2018) (cf. p. 39).

- Le dépôt de logiciel maintenant disponible sur HAL et tous les portails / CCSD. 25 sept. 2018. URL : https : //www . ccsd . cnrs.fr/2018/09/le-depot-delogiciel-maintenant-disponible-sur-hal-et-tous-les-portails/ (visité le 05/12/2018) (cf. p. 26).

- Refonte du module statistiques : résultats de l'enquête auprès des administrateurs de portail. 16 mai 2018. URL : https://www.ccsd.cnrs.fr/2018/05/refonte-dumodule-statistiques-resultats-de-lenquete-aupres-des-administrateursde-portail/ (visité le 17/05/2018) (cf. p. 74).

MaHÉ, Annaig. Bibliothèques et archives ouvertes. $1^{\mathrm{er}}$ jan. 2011. URL : http://bbf . enssib.fr/consulter/bbf-2011-01-0014-003 (visité le 16/04/2018) (cf. p. 25).

Mandat de dépôt. URL : http : //orbilu . uni . lu/project?locale=fr\&id=projectmandate (visité le 03/04/2018) (cf. p. 29).

Mandat pour l a diffusion en libre accès. 31 juil. 2017. URL : http://www. ifsttar.

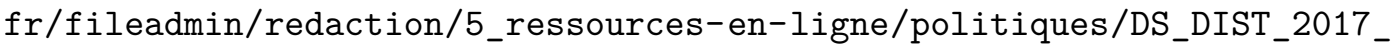
IFSTTAR_mandat_OA_et_depot_Madis.pdf (visité le 23/03/2018) (cf. p. 52).

MARIE FARGe et Frédéric HÉLEIN. Transition vers l'Accès Libre : le piège des accords globaux avec les éditeurs. 13 avr. 2018. URL : https : //blogs . mediapart.fr / edition/au-coeur-de-la-recherche/article/130418/transition-vers-1acces-libre-le-piege-des-accords-globaux-avec-les-editeur (visité le 24/04/2018) (cf. p. 31).

MarXiv. URL : https://marxiv.org/ (visité le 23/04/2018) (cf. p. 27).

MathOA - Converting mathematics journals to open access. URL : http://www mathoa. org/ (visité le 19/02/2018) (cf. p. 38). 
Mega journal. In : Wikipedia. Page Version ID : 822136229. 24 jan. 2018. URL : https: / / en . wikipedia . org / w / index . php ? title = Mega _ journal \& oldid $=822136229$ (visité le 09/03/2018) (cf. p. 39).

Melot, Michel. La sagesse du bibliothécaire. Sagesse d'un métier. Paris : l'Oeil neuf éd., 2004, 109 p. ISBN : 978-2-915543-03-2. URL : http : //www . sudoc .fr/077485920 (cf. p. 4).

MindRxiv. URL : https : //www . mindandlife.org/mindrxiv/ (visité le 23/04/2018) (cf. p. 27).

NARCIS. URL : https://www. narcis.nl/ (visité le 23/04/2018) (cf. p. 30).

Nidae ar-Ryadh / Appel de Ryadh - Libre accès à l'information scientifique et technique. Fév. 2006. URL : http://openaccess . inist. fr/?Nidae-ar-Ryadh-Appel-deRyadh (visité le 20/04/2018) (cf. p. 22).

Nouvelle ère pour les collections - Bibliothèques - Université de Montréal. URL : http: //www . bib. umontreal.ca/collections-nouvelle-ere/default.htm (visité le 19/04/2018) (cf. p. 20).

NutriXiv. URL : https : //osf . io/preprints/nutrixiv (visité le 23/04/2018) (cf. p. 27).

O'CArroll, Conor et Bernard Rentier. Evaluation of Research, Careers fully acknowledging, Open Science practices. Commission européenne, 2017. URL : https: //cdn1 . euraxess . org/sites/default/files/policy_library/os-rewards wgreport-final_integrated_0.pdf (visité le 19/02/2018) (cf. p. 44, 71).

Obligation de dépôt de la documentation Ifremer dans Archimer. URL : http://archim er.ifremer.fr/html/depot.htm (visité le 23/03/2018) (cf. p. 52).

Open Access - GTAO. URL : http://www.couperin.org/services-et-prospective/ open-access/gtao (visité le 19/02/2018) (cf. p. 52).

Open access : une position commune de la CDEFI, la CGE et la CPU. URL : http: //www.cdefi.fr/fr/actualites/open-access-une-position-commune-de-lacdefi-la-cge-et-la-cpu (visité le 11/04/2018) (cf. p. 70).

Open Access / Université de Rennes 1. URL : https://openaccess.univ-rennes1.fr/ (visité le 28/03/2018) (cf. p. 53, 57).

Open Access Button. URL : https://openaccessbutton.org/ (cf. p. 41).

Open Access et Evaluation de la Recherche. Oct. 2016. URL : https://openeval2016. sciencesconf.org/ (visité le 19/02/2018) (cf. p. 53, 55).

Open Access Explained! URL : http://phdcomics . com/tv/?v=L5rVH1KGBCY (visité le 19/02/2018) (cf. p. 23).

Open Access France - Le site couperin de l'accès ouvert en France. URL : http: // openaccess . couperin.org/ (visité le 19/02/2018) (cf. p. 52).

Open Access Policy concerning UNESCO publications. 31 juil. 2013. URL : http://www. unesco . org/new/fileadmin / MULTIMEDIA / HQ / ERI / pdf / oa_policy_rev2 . pdf (visité le 23/03/2018) (cf. p. 52).

Open Access Week: About. URL : http://openaccessweek.org/page/about (visité le 02/05/2018) (cf. p. 54).

Open Access.nl. URL : http://www. openaccess.nl/en (visité le 19/02/2018) (cf. p. 33).

Open Library of Humanities. URL : https: //www . openlibhums . org/ (visité le 19/02/2018) (cf. p. 38).

OpenAIRE - FAQ. URL : https://www .openaire.eu/faq (visité le 06/04/2018) (cf. p. 67$)$. 
OpenAPC : APC paid by publishers. URL : https://treemaps.intact-project.org/ apcdata/openapc/\#publisher/ (visité le 20/02/2018) (cf. p. 31).

OpenDOAR : About. URL : http://www . opendoar .org/about.html (visité le 20/02/2018) (cf. p. 28).

OpenDOAR : Charts - Worldwide. URL : http : //www . opendoar .org/find . php? format=charts (visité le 20/02/2018) (cf. p. 28).

OpenDOAR : Directory of Open Access Repositories. URL : http://www. opendoar.org/ (visité le 17/02/2018) (cf. p. 27).

OpenEdition. URL : http://www.openedition.org/ (visité le 08/03/2018) (cf. p. 34).

ORBi. URL : https://orbi.uliege.be/ (visité le 20/02/2018) (cf. p. 28).

ORBi : Contenu. URL : https : / / orbi . uliege . be / project ? id $=103$ (visité le 20/02/2018) (cf. p. 28).

OSF Preprints. URL : https://osf.io/preprints/ (visité le 23/04/2018) (cf. p. 26).

OuRY, Antoine. Finlande : Elsevier et les universités signent un accord pour plus d'accès ouvert. 23 jan. 2018. URL : https : //www . actualitte . com/article/mondeedition/finlande-elsevier-et-les-universites-signent-un-accord-pourplus-d-acces-ouvert/86895 (visité le 19/02/2018) (cf. p. 33).

- Un chiffre d'affaires de 8,3 milliards d'euros pour RELX (Elsevier) en 2017. 16 fév. 2018. URL : https://www .actualitte.com/article/monde-edition/un-chiffred-affaires-de-8-3-milliards-pour-relx-elsevier-en-2017/87344 (visité le 19/02/2018) (cf. p. 20).

Overlay journal. In : Wikipedia. Page Version ID : 771938195. 24 mar. 2017. URL : https : //en .wikipedia.org/w/index . php?title=0verlay_journal\&oldid=771938195 (visité le 09/03/2018) (cf. p. 39).

PaleorXiv. URL : https://paleorxiv.org/ (visité le 23/04/2018) (cf. p. 27).

Patterson, Mark. «Setting your cites on open : The Initiative for Open Citations ». JSO 2018. 24 jan. 2018. URL : https://jso2018. sciencesconf.org/data/pages/ Mark_Patterson.pdf (visité le 22/03/2018) (cf. p. 44).

PeerJ. In : Wikipedia. Page Version ID : 829790753. 10 mar. 2018. URL : https://en. wikipedia.org/wiki/PeerJ (visité le 25/04/2018) (cf. p. 39).

PeerJ - Open Access. URL : https://peerj.org/ (visité le 25/04/2018) (cf. p. 38).

Persée. URL : http://www.persee.fr/ (visité le 08/03/2018) (cf. p. 35).

Persée : À propos. URL : http://www.persee.fr/a-propos (visité le 08/03/2018) (cf. p. 35).

Persée : Historique. URL : http://info.persee.fr/historique/ (visité le 08/03/2018) (cf. p. 35).

PirOn, Florence. Guide de la recherche documentaire dans le web scientifique libre. 2018. URL : https: //www . projetsoha.org/?page_id=1040 (visité le 16/04/2018) (cf. p. 43).

PIWOWAR, Heather et al. « The state of OA : a large-scale analysis of the prevalence and impact of Open Access articles ». In : PeerJ 6 (13 fév. 2018), e4375. ISSN : 2167-8359. DOI : 10.7717/peerj.4375. URL : https://peerj.com/articles/4375 (visité le 05/12/2018) (cf. p. 31).

Plan national pour la science ouverte : discours de Frédérique Vidal - ESR : enseignementsuprecherche.gouv.fr. URL : http://www . enseignementsup-recherche.gouv.fr/cid 132531/plan-national-pour-la-science-ouverte-discours-de-frederiquevidal.html (visité le 06/07/2018) (cf. p. 4, 45). 
PLOS ONE : accelerating the publication of peer-reviewed science. URL : http://journ als.plos.org/plosone/ (visité le 09/03/2018) (cf. p. 38).

PLOS Open Letter. Sept. 2001. URL : https://www.plos.org/open-letter (visité le 20/04/2018) (cf. p. 21).

Politique de dépôt / Okina. URL : http://okina.univ-angers.fr/politique-dedepot (visité le 23/03/2018) (cf. p. 52).

Portail de revues scientifiques. URL : http://open.u-bordeaux.fr/journals/ (visité le 08/03/2018) (cf. p. 37).

Position de principe de l'Association of Learned and Professional Society Publishers (ALPSP) - Libre accès à l'information scientifique et technique. 27 août 2003. URL $: \mathrm{h}$ ttp://openaccess.inist.fr/?Position-de-principe-de-l (visité le 20/04/2018) (cf. p. 21).

Predatory open access publishing. In : Wikipedia. Page Version ID : 828044570.28 fév. 2018. URL : https://en.wikipedia.org/wiki/Predatory_open_access_publish ing (visité le 09/03/2018) (cf. p. 40).

Project factsheets / General Information. URL : https://www.openaire.eu/projectfactsheets (visité le 06/04/2018) (cf. p. 67).

Projet de décret 603 (2017-2018) visant à l'établissement d'une politique de libre accès aux publications scientifiques (Open Access). 7 mar. 2018. URL : http://archive. pfwb.be/1000000020870ee (cf. p. 29).

Projet de décret visant à l'établissement d'une politique de libre accès aux publications scientifiques (Open Access) - Parlement de la Fédération Wallonie-Bruxelles / Communauté française. 7 mar. 2018. URL : http://www.pfwb.be/le-travaildu-parlement/doc-et-pub/documents-parlementaires-et-decrets/dossiers/ 001591645 (visité le 23/04/2018) (cf. p. 29).

PsyArXiv preprints. URL : https://psyarxiv.com/ (visité le 20/02/2018) (cf. p. 27).

PsyOA. URL : http://psyoa.org/ (visité le 19/02/2018) (cf. p. 38).

Publier et diffuser / Aide à la publication - Coopérer en information scientifique et technique - Cirad. URL : https://coop-ist.cirad.fr/aide-a-la-publication/ publier-et-diffuser (visité le 28/03/2018) (cf. p. 53).

PubMed Central (PMC). URL : https : //www.ncbi.nlm.nih.gov/pmc/ (visité le 16/04/2018) (cf. p. 27).

Questions juridiques - HAL Documentation. URL : https://doc.archives-ouvertes. fr/questions-juridiques/ (visité le 20/02/2018) (cf. p. 26).

Redalyc. Sistema de Información Científica Redalyc. URL : http://www.redalyc.org/ home.oa (visité le 08/03/2018) (cf. p. 36).

Registry of Open Access Repositories. URL : http : / roar. eprints . org/ (visité le 16/04/2018) (cf. p. 28).

Rennes 1, Université de. Les revues prédatrices. 11 mai 2016. URL : https://openacc ess.univ-rennes1.fr/les-revues-predatrices (visité le 09/03/2018) (cf. p. 40, $62)$.

RePEc : Research Papers in Economics. URL : http://repec.org/ (visité le 16/04/2018) (cf. p. 27).

ResearchGate. URL : https://www.researchgate. net/ (visité le 12/03/2018) (cf. p. 40, $61)$.

Résolution votée par le Conseil scientifique de l'École normale supérieure sur l'accès libre aux publications. 17 juin 2016. URL : https://www. eleves.ens.fr/home/cacs/ 
documents-cs/2016-06-17/resolution-oa-ens.pdf (visité le 23/03/2018) (cf. p. 51).

Revues de Lyon 3. URL : https: //revues . univ-lyon3.fr/ (visité le 12/06/2018) (cf. p. 37).

Revues et Corpus en libre accès de l'Université Clermont Auvergne. URL : http: / / polen.univ-bpclermont.fr/revues_polen (visité le 08/03/2018) (cf. p. 37).

RIFKIN, Jeremy. La nouvelle société du coût marginal zéro l'internet des objets, l'émergence des communaux collaboratifs et l'éclipse du capitalisme. Trad. par Françoise Chemla et Paul Chemla. 2014. 1 vol. (509 p.) ISBN : 979-10-209-0141-5 (cf. p. 21).

Roarmap. Registry of Open Access Repository Mandates and Policies (ROARMAP). 2018. URL : http://roarmap.eprints.org/ (visité le 17/02/2018) (cf. p. 51).

RoORYCK, Johan. "Vers un nouveau modèle de publication : Fair Open Access ». JSO 2018. 23 jan. 2018. URL : https ://jso2018. sciencesconf .org/data/pages / Paris_Couperin23jan18.pdf (visité le 08/03/2018) (cf. p. 38).

Salvador Declaration on Open Access : the developing world perspective. 23 sept. 2005. URL : http://www.icml.org/public/documents/pdf/en/Dcl-Salvador-OpenAcc ess-en.pdf (visité le 20/04/2018) (cf. p. 22).

SCHIERMEIER, Quirin. Hundreds of German universities set to lose access to Elsevier journals. 5 déc. 2017. URL : http://www . nature .com/articles/d41586-01707817-1 (visité le 19/02/2018) (cf. p. 20).

Schimmer, Ralf. « Leveraging Bibliodiversity : Transforming the Journal System and Shifting our Spending from Subscription to Open Access ». JSO 2018. 23 jan. 2018. URL : https://jso2018. sciencesconf.org/data/pages/Ralf_Schimmer_2.pdf (visité le 09/03/2018) (cf. p. 33).

Sci-Hub. In : Wikipédia. Page Version ID : 146185697. 7 mar. 2018. URL : https :// fr . wikipedia . org/w/index . php?title=Sci-Hub\&oldid=146185697 (visité le 12/03/2018) (cf. p. 42).

Sci-Hub / Academic Search Engine - To remove all barriers in the way of science. URL : http://sci-hub.tech/ (visité le 12/03/2018) (cf. p. 42).

Sci-Hub Stats Browser. URL : https://greenelab.github.io/scihub/\#/ (visité le 19/02/2018) (cf. p. 42).

«Sci-Hub, le Pirate Bay de la recherche scientifique, est de retour ». In : Le Monde.fr (15 fév. 2016). ISSN : 1950-6244. URL : http://www.lemonde.fr/pixels/article/ 2016/02/15/sci-hub-le-pirate-bay-de-la-recherche-scientifique-estde-retour_4865611_4408996.html (visité le 14/03/2018) (cf. p. 42).

SciELO.org - Scientific Electronic Library Online. URL : http://www.scielo.org/php/ index.php (visité le 08/03/2018) (cf. p. 36).

Science Europe - cOAlition S. URL : https://www.scienceeurope.org/coalition-s/ (visité le 07/09/2018) (cf. p. 67).

Science, Technology and Innovation for the 21st Century. Meeting of the OECD Committee for Scientific and Technological Policy at Ministerial Level, 29-30 January 2004Final Communique - OECD. 30 jan. 2004. URL : http://www. oecd.org/science/sc $i-t e c h / s c i e n c e t e c h n o l o g y a n d i n n o v a t i o n f o r t h e 21$ stcenturymeetingof theoec dcommitteeforscientificandtechnologicalpolicyatministeriallevel29-30j anuary2004-finalcommunique.htm (visité le 20/04/2018) (cf. p. 22).

SciPost. URL : https://scipost.org/ (visité le 22/03/2018) (cf. p. 43). 
SELL signe une déclaration commune concernant l'open access. 5 juin 2018. URL : https: //www . couperin.org/breves/1342-le-sell-signe-une-declaration-communeconcernant-1-open-access (visité le 06/09/2018) (cf. p. 23).

Shamash, Katie. Article processing charges (APCs) and subscriptions : Monitoring open access costs. 27 juin 2016. URL : https://www.jisc.ac.uk/reports/apcs-andsubscriptions (visité le 20/02/2018) (cf. p. 32).

Sherpa Juliet. URL : http://v2 . sherpa .ac.uk/juliet/ (visité le 23/03/2018) (cf. p. 51).

SHERPA/RoMEO - Publisher copyright policies 8 self-archiving. URL : http://www . sherpa.ac.uk/romeo/index.php (visité le 19/02/2018) (cf. p. 59).

SIGMA (Symmetry, Integrability and Geometry : Methods and Applications). URL : htt p://www.emis.de/journals/SIGMA/ (visité le 09/03/2018) (cf. p. 39).

Sistema nacional de ciencia, tecnologia e innovacion. 3 déc. 2013. URL : http://rep ositorios . mincyt . gob.ar/pdfs/Boletin_Oficial_Ley_26899 .pdf (visité le 25/04/2018) (cf. p. 30).

Sistema Nacional de Repositorios Digitales - República Argentina. URL : http://repos itorios.mincyt.gob.ar/recursos.php (visité le 25/04/2018) (cf. p. 30).

SocArXiv papers : Open archive of the social sciences. URL : https://osf.io/preprin ts/socarxiv (visité le 20/02/2018) (cf. p. 27).

SportRxiv. URL : https://osf.io/preprints/sportrxiv (visité le 23/04/2018) (cf. p. 27).

Stop Predatory Journals. URL : https: //predatoryjournals. com/ (visité le 09/03/2018) (cf. p. 40, 62).

Stratégie nationale suisse Open Access. 31 jan. 2017. URL : https://www.swissuniver sities.ch/fr/themes/politique-des-hautes-ecoles/open-access/ (visité le 30/04/2018) (cf. p. 33).

Suber, Peter. History of open access. URL : https : / cyber . harvard.edu/ psuber / wiki/History_of_open_access (visité le 24/04/2018) (cf. p. 25).

- Open Access Overview : Focusing on open access to peer-reviewed research articles and their preprints. 21 juin 2004. URL : http://legacy.earlham.edu/ peters/ fos/overview.htm (visité le 19/02/2018) (cf. p. 25).

Susi, Toma. Finland takes steps in the openness of academic journal pricing. 25 jan. 2018. URL : https : / / www . mostlyphysics . net/blog/2018/1/25/finlandtakes-a-step-back-in-the-openness-of-academic-journal-pricing (visité le 08/03/2018) (cf. p. 33).

Textes de références - Libre accès à l'information scientifique et technique. URL : http: //openaccess . inist.fr/?-Textes-de-references- (visité le 19/02/2018) (cf. p. 21).

The Center for Open Science. URL : https://cos.io/ (visité le 23/04/2018) (cf. p. 26). «The scientists encouraging online piracy with a secret codeword». In : BBC News

(21 oct. 2015). URL : http : / / www . bbc . com/news / blogs - trending- 34572462 (visité le 14/03/2018) (cf. p. 42).

Toul'AO / Toulouse Archives Ouvertes. URL : http://openarchiv.hypotheses.org/ (visité le 19/01/2018) (cf. p. 53, 57).

Transférer le dépôt vers arXiv - HAL Documentation. URL : https://doc.archivesouvertes.fr/deposer/transfert-hal-arxiv/ (visité le 04/04/2018) (cf. p. 63).

Twitter : ICanHazPDF. URL : https://twitter.com/hashtag/icanhazpdf (cf. p. 42). 
Une déclaration de principe du Wellcome Trust en soutien à l'édition en Libre Accès - Libre accès à l'information scientifique et technique. Nov. 2003. URL : http:// openaccess.inist.fr/?Une-declaration-de-principe-du (visité le 20/04/2018) (cf. p. 22).

Unpaywall. URL : http://unpaywall.org/ (visité le 19/02/2018) (cf. p. 41).

VAJOU, Michel. «Une étude britannique met en lumière les effets secondaires négatifs des « big deals » associant négociation des budgets d'abonnements et des budgets d'APC ». In : EPRIST Analyse I/IST 24 (nov. 2016). URL : http://www. eprist. fr/wp-content/uploads/2016/11/I-IST_24EtudeJISC.pdf (visité le 20/02/2018) (cf. p. 32).

Visibilite des archives ouvertes. URL : https : //visiarchives . sciencesconf .org/ (visité le 06/12/2018) (cf. p. 56).

Visibilité des dépôts HAL : moissonnage, signalement - HAL Documentation. URL : https : //doc . archives-ouvertes .fr/guide_utilisateurs/visibilite-desdepots-hal-moissonnage-signalement/ (visité le 04/04/2018) (cf. p. 58).

Washington D.C. Principles For Free Access to Science : A Statement from Not-forProfit Publishers. 16 mar. 2004. URL : http://www.dcprinciples.org/statement. pdf (visité le 20/04/2018) (cf. p. 22).

WillO. URL : https://decadoc.typeform.com/to/W2ZZMV (visité le 28/03/2018) (cf. p. 53,60$)$.

Zenodo - Research. Shared. URL : https ://www . zenodo.org/ (visité le 06/04/2018) (cf. p. 67). 


\section{Table des matières}

$\begin{array}{ll}\text { Préface } & 3\end{array}$

$\begin{array}{lr}\text { Sommaire } & 7\end{array}$

$\begin{array}{ll}\text { Synthèse } & 9\end{array}$

$\begin{array}{ll}\text { Avant-propos } & 15\end{array}$

$\begin{array}{ll}\text { Remerciements } & 17\end{array}$

$\begin{array}{ll}\text { Introduction } & \mathbf{1 9}\end{array}$

Un coût qui n'est plus soutenable . . . . . . . . . . . . . . . . . . 19

Un modèle économique obsolète . . . . . . . . . . . . . . . . . . . . . . 20

Un grand intérêt pour l'OA . . . . . . . . . . . . . . . . . . . 21

1 Open Access : de quoi parle-t-on? 25

1.1 Green $\mathrm{OA}$ : la voie verte . . . . . . . . . . . . . . 25

1.1.1 Archives ouvertes . . . . . . . . . . . . . . 25

1.1.2 Preprints et postprints . . . . . . . . . . . . . . . 26

1.1.3 Archive ouverte thématique, nationale ou institutionnelle? . . . . 26

1.1.4 La voie verte dans le monde . . . . . . . . . . . . . . . . . 28

1.2 Gold OA : la voie dorée . . . . . . . . . . . . . . . . . . . 30

1.2.1 Auteur-payeur et APC . . . . . . . . . . . . . . . . . 30

1.2.2 Bronze OA : la forme dégradée du Gold . . . . . . . . . . . . . . 31

1.2.3 La transition dorée vers l'OA ? . . . . . . . . . . . . 31

1.3 Diamant et platine : l'édition électronique en OA . . . . . . . . . . . 34

1.3.1 Les grands éditeurs publics . . . . . . . . . . . . . . . . . 34

1.3.2 Les sociétés savantes : EDP Sciences . . . . . . . . . . . . 35

1.3.3 Quelques projets étrangers . . . . . . . . . . . . . . . 36

1.3.4 Les revues publiées par les universités . . . . . . . . . . . . . 36

1.3.5 Le Fair Open Access . . . . . . . . . . . . . . . . . . . . . . 37

1.4 D'autres formes de revues . . . . . . . . . . . . . . . 38

1.4 .1 Les mégarevues . . . . . . . . . . . . . . . . . . . 38

1.4 .2 Les épi-revues . . . . . . . . . . . . . . . . . . . . . . . . 39

1.4.3 Les revues prédatrices . . . . . . . . . . . . . . . . . 39

1.5 Grey : la zone grise . . . . . . . . . . . . . . . . . . 40

1.5.1 Les réseaux sociaux académiques . . . . . . . . . . . . 40

1.5.2 Unpaywall . . . . . . . . . . . . . . . . . . . . . . . . 44 4

1.5 .3 Lazy Scholar . . . . . . . . . . . . . . . . . . . . 41 
1.5.4 Open Acces Button . . . . . . . . . . . . . . . . . 4 41

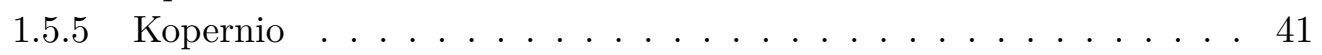

1.6 Black : les solutions illégales . . . . . . . . . . . . . . . . . . . . 41

1.6.1 \#ICanHazPDF . . . . . . . . . . . . . . . . . . 42

1.6 .2 Sci-Hub . . . . . . . . . . . . . . . . . 42

1.6 .3 LibGen . . . . . . . . . . . . . . . . . . . . . 42

1.7 De l'Open Access à la Science ouverte . . . . . . . . . . . . . . . . . . . 43

1.7.1 Travail collaboratif sur les preprints . . . . . . . . . . . . 43

1.7.2 Publication des données de la recherche . . . . . . . . . . . . . 43

1.7.3 Citation ouverte . . . . . . . . . . . . . . . . . 44

1.7.4 Evaluation ouverte . . . . . . . . . . . . . . . 44

$\begin{array}{lll}2 & \text { Etat des lieux } & \mathbf{4 7}\end{array}$

2.1 HAL et les archives ouvertes institutionnelles . . . . . . . . . . . . . 47

2.2 Initiatives académiques en faveur de l'OA . . . . . . . . . . . . 50

2.2 .1 Mandat de libre accès . . . . . . . . . . . . . . . . . . . 51

2.2 .2 Services autour de l'OA . . . . . . . . . . . . . . . . . 52

2.2 .3 Financement de l'OA . . . . . . . . . . . . . . . 54

2.3 Manifestations à l'occasion de l'Open Access Week . . . . . . . . . . . . 54

2.3.1 Une information dispersée . . . . . . . . . . . . . . . 54

2.3.2 Les manifestations $2017 \ldots \ldots \ldots \ldots \ldots$

2.4 Journées d'étude . . . . . . . . . . . . . . . . . . . . . . 55

$\begin{array}{llr}3 & \text { Obstacles } & \mathbf{5 7}\end{array}$

3.1 C'est quoi, les archives ouvertes ? . . . . . . . . . . . . 57

3.2 Y ai-je intérêt $\ldots \ldots \ldots \ldots \ldots \ldots$

3.2 .1 Je ne veux pas divulguer ma production sans contrôle . . . . . . 58

3.2 .2 Est-ce bon pour ma carrière ? . . . . . . . . . . . . . 58

3.2.3 La publication en OA n'offre pas la garantie de qualité de l'édition commerciale . . . . . . . . . . . . . . 5 58

3.2.4 HAL est encore un de ces machins franco-français : je préfère déposer mes publications sur Academia ou ResearchGate pour avoir une vraie visibilité internationale . . . . . . . . . . . . 58

3.3 Ai-je le droit $\ldots \ldots \ldots \ldots \ldots$. . . . . . . . . . . . . . . . . . 59

3.3.1 La loi permet le dépôt en archive ouverte . . . . . . . . . . . 59

3.3.2 Des outils pour déterminer le droit . . . . . . . . . . . . . 59

3.4 C'est trop compliqué / Je n'ai pas le temps . . . . . . . . . . . . . . . . 60

3.5 Je trouve déjà ce que je cherche ailleurs . . . . . . . . . . . . . . 60

3.5.1 Je trouve déjà presque tout ce que je cherche sur gratuitement sur Internet . . . . . . . . . . . . . . . 60

3.5.2 Je trouve déjà ce que je cherche sur Academia / ResearchGate . . 61

3.5.3 Unpaywall / Lazy Scholar / Open Access Button / Kopernio me permet de trouver gratuitement ce que je cherche . . . . . . . . 61

3.5.4 Il suffit de demander sur Twitter avec le hastag \#IcanHazPDF . . 62

3.5.5 Il y a tout sur SciHub et LibGen . . . . . . . . . . . . . . . . 62

3.5.6 Je publie déjà dans des revues en $\mathrm{OA} \ldots$. . . . . . . . . . . 62

3.6 Je ne sais pas où déposer mes publications . . . . . . . . . . . . . . 63

3.6 .1 HAL . . . . . . . . . . . . . . . . . 63 
3.6.2 Archive ouverte institutionnelle . . . . . . . . . . . . . . . 63

3.6 .3 Archive ouverte thématique . . . . . . . . . . . . . 63

3.7 J'aimerais publier dans une revue en OA, mais je ne sais pas comment m'y prendre . . . . . . . . . . . . . . . . . . . . 64

\section{Propositions}

4.1 Communiquer largement sur les objectifs et possibilités de diffusion des travaux de la recherche . . . . . . . . . . . . . . . . 65

4.1 .1 Le code de la recherche . . . . . . . . . . . . . . . . 65

4.1 .2 Les recherches subventionnées H2020 . . . . . . . . . . . . . 67

$4.1 .3 \quad$ cOAlition S et le Plan S . . . . . . . . . . . . . . . . 67

4.1.4 Les initiatives académiques en faveur de l'OA . . . . . . . . . 67

4.2 Rendre la publication en OA incitative pour la carrière des chercheurs . 67

4.2.1 Le Conseil national des universités (CNU) . . . . . . . . . . . 68

4.2.2 Le Haut Conseil de l'évaluation de la recherche et de l'enseignement supérieur (HCERES) _ . . . . . . . . . . . 68

4.2.3 L'Agence nationale de la recherche (ANR) . . . . . . . . . . . . . 69

4.2.4 Les conférences d'établissements (CPU, CDEFI, CGE) . . . . . . . 69

4.2.5 Le Comité pour la Science Ouverte (CoSO $\ldots \ldots$. . . . . . . . 70

4.2 .6 La Commission européenne . . . . . . . . . . . . . . . . . 71

4.3 Accélérer la prise en compte de l'OA par les établissements . . . . . . . . 71

4.3.1 Lancer un appel à projet MESRI pour un label . . . . . . . . . . . 71

4.3.2 Rendre la définition d'une politique d'OA obligatoire pour les établissements d'enseignement supérieur et de recherche et les organismes de recherche . . . . . . . . . . . . . . 72

4.4 Renforcer les services communs de documentation (SCD) dans leur rôle d'acteur du développement de l'OA . . . . . . . . . . . . . . . . 72

4.4.1 Soutenir les SCD dans leur rôle d'accompagnateur au développement de l'OA . . . . . . . . . . . . . . . . . . . 72

4.4.2 S'appuyer sur les SCD pour garantir la qualité des métadonnées et des référentiels . . . . . . . . . . . . . . . 73

4.5 Ajouter des fonctionnalités complémentaires à HAL . . . . . . . . . . . 73

4.5.1 Introduire des savoir-faire répandus dans le monde des bibliothèques pour la gestion des référentiels . . . . . . . . . . . 73

4.5.2 Produire les données de l'ESGBU avec le module statistique de HAL 73

4.5.3 Faire une revue systématique de l'interface de HAL . . . . . . . . . 74

4.6 Créer une plateforme fédératrice de l'action des bibliothèques pour l'Open Access en France . . . . . . . . . . . . . . . . . . . . . . . . . . . 74

4.6 .1 Porte d'entrée. . . . . . . . . . . . . . . . . . . . 74

4.6 .2 Observatoire . . . . . . . . . . . . . . . 75

4.6 .3 Intégration et portage $\ldots \ldots \ldots \ldots \ldots$. . . . . . . . 75

$\begin{array}{lr}\text { Conclusion } & 77\end{array}$

$\begin{array}{ll}\text { Résumé des propositions } & \mathbf{7 9}\end{array}$ 
A Données : recensement des archives ouvertes des établissements d'enseignement supérieur et de recherche et des organismes de recherche 83 Méthode de constitution du corpus . . . . . . . . . . . . . . . 83

Etablissements d'enseignement supérieur et de recherche . . . . . . . . . 83

Organismes de recherche . . . . . . . . . . . . . . . . . . 84

Protocole . . . . . . . . . . . . . . . . . . . 8 85

A.1 Universités . . . . . . . . . . . . . . . . . . . . . 85

A.1.1 Universités à statut ordinaire . . . . . . . . . . . . . . . 85

A.1.2 Universités à statut dérogatoire . . . . . . . . . . . . . . . . . 94

A.2 ComUEs et associations . . . . . . . . . . . . . . . . . . 94

A.2.1 ComUEs . . . . . . . . . . . . . . . . . . . . . . 94

A.2.2 Associations. . . . . . . . . . . . . . . 96

A.3 Instituts et écoles extérieurs aux universités . . . . . . . . . . . . . . . 96

A.3.1 Ecoles . . . . . . . . . . . . . . . . . . 96

A.3.2 Instituts . . . . . . . . . . . . . . . . . . . . . . . 998

A.3.3 Universités de technologie . . . . . . . . . . . . . . . . . . 98

A.4 Ecoles normales supérieures . . . . . . . . . . . . . . . . . . . . 99

A.5 Grands établissements . . . . . . . . . . . . . . . . . . . . . 99

A.5.1 Article D717-1 : tutelle ESR . . . . . . . . . . . . . . . . . 99

A.5.2 Article D717-2 : tutelle mixte . . . . . . . . . . . . . . . . 101

A.5.3 Article D717-3 : autres tutelles . . . . . . . . . . . . . . 102

A.6 Ecoles nationales (supérieures) d'ingénieurs . . . . . . . . . . . . . . 104

A.7 Instituts d'études politiques . . . . . . . . . . . . . . . . . . . 104

A.7.1 IEP à statut ordinaire . . . . . . . . . . . . . . . . . 104

A.7.2 IEP à statut particulier . . . . . . . . . . . . . . . 105

A.8 Organismes de recherche . . . . . . . . . . . . . . . . . 105

A.9 Bibliothèque nationale de France . . . . . . . . . . . . . . . . 110

B Données : recensement de l'OA week $2017 \quad 111$

Méthode de constitution du corpus . . . . . . . . . . . . . . . . . . 111

Evénements . . . . . . . . . . . . . . . . . . . . . . . . 112

$\begin{array}{ll}\text { C Extrait des documents stratégiques de l'Enssib } & 117\end{array}$

C.1 Contrat de site 2016-2020 Université de Lyon : volet spécifique Enssib . . 117

C.2 Projet d'établissement Enssib 2020 . . . . . . . . . . . . . . . . 117

Glossaire

Bibliographie

$\begin{array}{ll}\text { Table des matières } & 135\end{array}$ 



\section{enssib"}

17-21, boulevard du 11 novembre 1918

69623 Villeurbanne Cedex

$+33(0) 472444343$

www.enssib.fr 